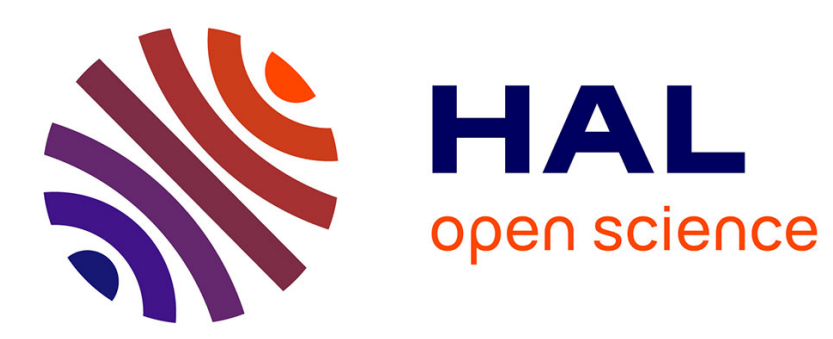

\title{
Simplicités, empirisme et interprétation psychologique dans la grammaire générative.
}

Gabriel G. Bès

\section{To cite this version:}

Gabriel G. Bès. Simplicités, empirisme et interprétation psychologique dans la grammaire générative.. Archives et documents de la société d'histoire et d'épistémologie des sciences du langage, 1981, 1, pp.1-84. hal-01064415

\section{HAL Id: hal-01064415 https://hal.science/hal-01064415}

Submitted on 16 Sep 2014

HAL is a multi-disciplinary open access archive for the deposit and dissemination of scientific research documents, whether they are published or not. The documents may come from teaching and research institutions in France or abroad, or from public or private research centers.
L'archive ouverte pluridisciplinaire HAL, est destinée au dépôt et à la diffusion de documents scientifiques de niveau recherche, publiés ou non, émanant des établissements d'enseignement et de recherche français ou étrangers, des laboratoires publics ou privés. 


\section{ARCHIVES ET DOCUMENTS \\ de la \\ SOCIETE D"HISTOIRE ET D'EPISTEMOLOGIE \\ DES SCIENCES DU LANGAGE}

$$
\mathbb{N}^{\circ} \quad 1
$$

GABRIEL G. BES

Simplicités, empirisme et interprétation psychologique dans la gramiaire générative

SAINT-CLOUD

S.H.E.S.L. 


\title{
ARCHIVES ET DOCUMENTS
}

\author{
DE LA \\ SOCIETE D*HISTOIRE ET D'EPISTEMOLOGIE
} DES SCIENCES DU LANGAGE

$$
\text { (S.H.E.S.L) }
$$

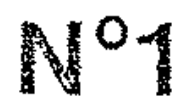

Aaministration, Th. OLIU- Ecole Normale Supérieure, Section d'Espagnol, Paxe de Saint-Clond,Grille d'honneur-92211 Saint-Cloud.

REdaOtion, S. AUROUX, J AUTHYER, J. J . COURTINE, A. GRESILION, C1 . HAROCHE, P . HENRY, D. MALDIDIER, T. SOUBLIN.

Toute cortespondance ou manuscrit doit etre adressze a C1. HAROCHE, 85 rue d'Assnsparis 75006 mes nanuscrits regus seront sounis au comité de lacture selon une procëcre rapide. Loxsqu'tu texte sera accapta, il sera demande aux auteurs'd'en fournir whe version directenent reproductible en offset. 


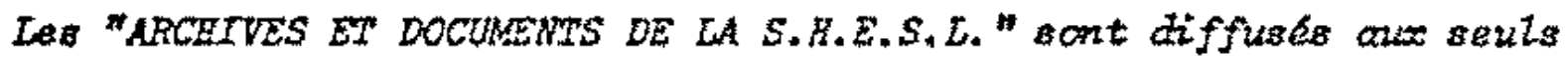
adisererts de Za S.R.E.S.L.

Les raisans de leur Lancement sont clairea: Za "Socibte d'Bistoire et

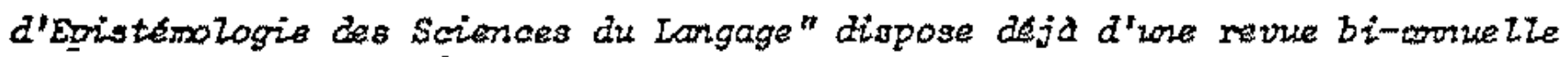
(comercialised qui ne comporta qu'ute containe de paged por livraison. Il now a paru interssant d'elargir les possibilités de publication et

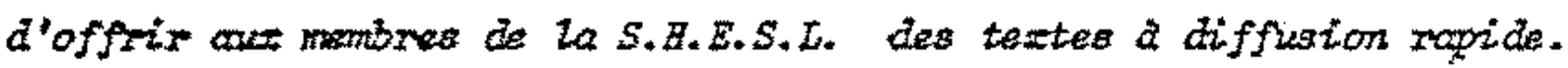
EQS "ARCDTYES ET DOCURENSS DE LA S.H.E.S.L*" $n$ 'etant pas comerciazists, et faisant l'abjet d'use diffusion reatreinte, on ana compris qu'il s'agit de "Woxking popers".

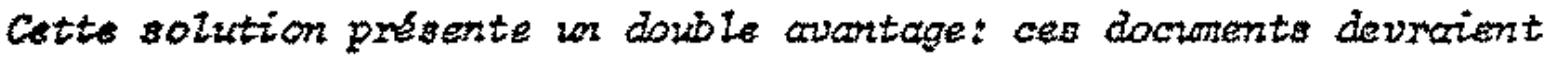

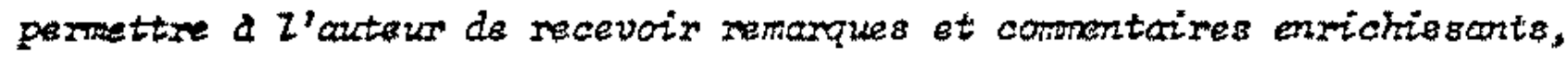

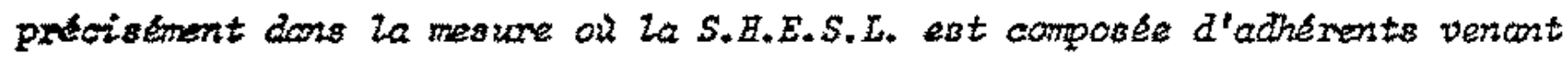
dhorizans tres divers. Le leatew, de son cote, pourna ae trower confrante d Z'expose de points do vue auquels it peut ne pas etre fomiliorise. Pandonentalement, it s'ogit ict do favomiser echonges et discusaions, en un not d'oenoner an decloisonnenent de disciplines wivereitaires qui ont parfois

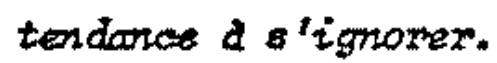

How sowhaterions susciter a côté des clemarches plus ou moina tradition nelles et parfois circonspectes, des vues theoriques tot peu audacieuses et stimulantes, en partioulier en ce qui conceme l'Epitiénologie, la Theorie et l'Bistoire, et incitor ainst las auteurs a prentre parfois le risque de sortiz des goraties ot des aertitudze qu'offre un paeitivisme de bon atoi. 
SIMPLTCITES, EMPIRISME ET INTERPRETATION PSYCHÜLOUTUL

DANS LA GRARAATRE GERERATIVE

G.G.BES

La publication de ce document a eté permise grace a we subvantion de l'amicale des anciens elteves de l'Ecole Nomale stos rieure de Saint-Claü." 
plus ae deux decenries après la publication des premiers écrits de Noam chomsky sur la grammante genêrạtive, une douzain d'années après qu'elle eut eté introduite en France( $)$ et alors que sa notation et sa terminologie se répandent jusque dans les textes scolaires, on peut et on doit se poser des questions sux ce molvement linguistigue gij apparait aujourd'hui comme un des plus importants si lon considure les signes externes du"pouvotr scientifique" : nombre et importance des publications, répexcussion sur les aisciplines connexes, interêt soulevé chez les jeunes chercheurs, appropration des concepts-ou de la terminologiem par le milieu culturel, etc..Il semble bien que les questions qui doivent etre posés sont les sulvantes:Est-ce que la grammaire gênerative a rếllement avancé dans la connaissance du langage? si oui, quelles sont les donnés nouvelles qu'elle a mises en Iumiere?quelles sont celles qu'elle a expliquees? Bref, quel est cet ensemble a'acquis sur le langage que l'on pourrait considérer désormais come suftisamment sürs pour qu'on puisse penser qu'ils ne seront pas renis en doute tres bientot?

Si I'on veut eviter les exreurs souvent commises au monent de la publication des premiers ecrits generativistes-rejet total ou adnesion non critique- il est impossibie de repondre aux questions precedentes de maniexe simpliste ou globale: il est vaisemblable que le bllan final ne se fera pas pax ouj ou par non, mais que chague aspect examine exigera une rêponse particulière et nuancëe. ce travail, centre sur la notion de simplicite en gramnatre genem. rative, s'inscrit dans cette perspective de fond de contribuex à dresser, de manièxe documentëe et raisonnee, un bilan des apports de ce mowvenent, qui deborde du linguistigue sux le psychologigue et le pintosophique.

Le vocabulajxe mëthodologicue employe ici sera très simple. Nous reconnaissons deux types de sciences: les sciences empiriques et les sciences formelies. 
On aira qu'une proposition est une hypothesc uppixique, ol piss simplement une hypothèse, si, par rapport a des donnops dnnt elle doit rendre compte de maniere directe ou indirecte, on peut dire cu'elie est vraje ou fausse.on dira d'une science qu'elle est empirique si elie est composee d'hypothèses empiriques. En revanche une sclence formelle $n$ 'est pas composee d'hypotheses empiriques; elle ne possede pas des conditions d'adequation par rapport a des données externes, les propositions qui l'intègrent se valident en fonction de critères internes.

Dans ce cadre de discussion, empirique ne veut donc dire ni "doue d'une existence perceptible", ni" constate dans les falts mais non explique theoriquement", ni "ontologiquement reel", ni"formulé a la suite de l'observation du réel et par abstractions successives? Dans ce travail, empirique voudra simplement dire: proposition susceptible d'tetre testée par des donnëes. On ne prejuge rien sur le fait que ces donnes soient percoptibles ou non, qu'elles snient susceptibles d'être produites et reproduites dans des conditions controlables ou non, qu'elles aient etê observees avant de degager l'hypothese correspondante ou non. Toutes ces possibilités restent ouvertes dans la caractérisation ici adoptee d'empirlque. (2).

Il sera parfols nécessaire d'introduire une distinction entre science entièrement empirique et science partiellement empirique.une science entierement empirique est celle qui est entierenent composee d'hypotheses empiliytus, nul critire methodologique autre que les exigences sur l'explicitation et la cohérence des hypothèses n'etant employe pour sêlectionner celles-ci. science partiellement empirique sera celle qui est composée a'hypothèses empiriques mais qui est completêe par des formulations methodologiques gênerales, non empiriques, destinteg à choisir entre plusieurs hypotheses possibles. Au cours au developpement des sciences, on a alcribue a la simplutits ce role jarbitrage ner. empixique entre hypothèses en concurrence sur un môme domaine. 
L'interprétabilite psychologlque d'une throrie ou ensemble d'hypothèses sur le langage concerne le problème posé par ce qu'on appelle couramment la réalitê psychologique de la thérie ou son conténu psychologique.ce problène peut utilement être posé à l'aide des notions de modèle de boite noire et de modèle de boite transparente.sur un plan général, on dira qu'une thuile ou ensemble d'nypothèses emplriques, comprenant dans celles-ci les déductions particulières qu'on peut tirer des formulations gênérales, peut être associēe soit au comportement d'un objet et, dans ce cas, on a un modele de borte noire, soit aux parties constitutives de l'objet, en général non observables, et à son comportement extê- . rieur; dans ce deuxième cas, on a un modèle de boite transparente. Dans le domaine du langage, on dira qu'une theorie ou hypothèse empirique est interprétable psychologiquement où, si l'on préfère qu'une théorie ou hypothèse possède une réalité psychologique si, non seulement les déductions faites à partir de la théorie ou de I'hypothèse sont associables à un comportement extérieur, mais si également, les parties constitutives de cette theorie ou de cette hypothèse sont elies-mêmes censées être associées aux parties constitutives internes de l'organisation mentale qui est responsable de ce comportement extêrieur. (3).

Nous supposons que, sur un plan général, la notion de simplicité est une notion acceptée et connue intuitivement.elie est susceptible, dans une formulation générale, d'être ramenée à une relation quantitative:A est pius simple que B si A possède moins d'éléments de type x que B.Etre plus simple signifie, par consêquent, être doué de moins d'éléments. Ce sont les entités $A$ et $B$, ou le trait $x$, ou l'échelle par rapport à laquelle on détermine le plus et le molns, qui vont être modifiés dans chaque formulation particulière; mais, dans tous les cas, réapparàt le même schẹma gênéral.

Le statut de la simplicité n'est pas clair dans la methodologie scientifique. Une simplicité formelle, d'explicitation difficile, a été paxfois utilisée pour choisir entre plusieurs hypothèses empiriques possibles, d'où il résulte une situation quelque peu 
paxadoxale: dans I'organisation des hypothèses d'une science empirique apparait un element qui ne l'est pas(4). Mais une formulation de simplicite- ou plus simplement, une simplicité$n^{*} a$ pas comme seule possibilité celle d'etre formelle; en fait, rien ne s'oppose, en principe, à ce qu'une simplicité soit empixique bans ce cas, ce qui a eté dêclaré conme pius (ou moins) simple doit etre associé à une donnée qui peut valider ou infirmer la proposition de simplicité.par exemple, si l'on choisit entre hypothèses en concurrence par le biais de la simplicité, cellemci sera une simplicite formelle si l'hypothèse choisie comre plus simple ne possède aucun type de vérification empirique susceptible de la distinguer de la moins simple; en revanche, la simplicité elle-meme sera empirique si l'on exige de l'hypothèse choisie comme plus simple qu'elle possède certains traits d'adéquation externe, absents de la moins simple.une simplicité empirique est, par conséguent une hyoothèse à paxt entière, de laquelle on doit pouvoir dire si elle est vraie ou fausse.par ailleurs, une simplicité empiriq-ue, dans le domaine du langage, peut ou non être interprêtëe psychologisuement. Elle aura une interprêtation psychologique si elle est censée être associée à un mécanisme interne, mental, qui agit au cours des conduites linguistiques.

Le dilemne science formelle vs.science empirique est apparu dans la linguistique post-saussurienne, sans qu'il ait éte ni clairement posé ni tranche.

En effet, la Iinguistique a ete considêrée parfois comme une : science empirique parfois comme une science formelle. Saussure et Troubetzkoy la conçoivent comme science empirique de même que; par exemple, Martinet.En revanche, dans queIques secteurs du structuralisme americain, on cherche à valider des analyses Iinguistiques selon leur adequation par rapport à une procédure analytique dêterminêe, fondēe elie-même sur des dêfinitions et des mêthodes posées au préalable; dans cette optique, une description Iinguistique se valide moins par sa capacite de rendre compte des 
donnes que par ze falt d'avoir applique de manière cohénente un certain formalisme descriptif qui, lui-meme, n'est pas enpirigue (5).

W'ambiguate methodologique de la linguistique appaxalt dans toute son intensite avec Hjelmslev. Celui-ci soulève bien le probleme du rapport de la theorie avec les donnés, mais il aboutit une conclusion difficile à comprendre.

Il affirme (56b P.28-29/56 p.14-15), en effet:

"1-La theorie ellememe ne depend pas de 1 experience.Rien en elle n'indique si elle aura des applications en rapport avec ces donnés de 1 'expexience. Elle ne postule nullement 1 'existence de ces données. Elie constitue ce que l'on peut appelex un pur systeme déductif, en ce sens gue c'est la théorie à elle seule qui, a partir des premisses qu'elle enonce, permet lo caicul des possibilitês qui en résultert.

2-Le theoricien sait d'experience que certaines prenisses Enoncées dans la théorie remplissent les conditions nécessaires pour que cellemi sait applicable à certains faits".

La prenzìne de ces caracteristiques constitue, d'apres lui, le caractèxe arbitzaire de la theorie; la deuxième, sor caractèxe approprié. Les donness ne peuvent jamais "ni confirmer ni infirmer la validite de la thérie". Sa conclusion en est que "la question déja soulevê du rapport entre theorie et réalité aura une reponse double: en vertu de son caractere arbitraite, la théorie est

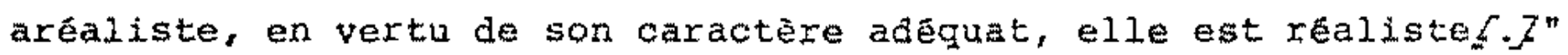

si par "arbitraire" on comprend formel et empirique par "approprie" , la conculusion de Hjelmslev enferme lane contradiction impossible à cepasser: une thérie ne peut à la fois être vërifiable et non véfifiabie par des donneses.Cependant, la contradiction pourrait être levee si l on assignait a "applicabijite" non pas le sens de "confrontation avec les donnees pour tester les hypotheses" mais le sens de "determination des possibilites de la theorie qui sont attestêes sur le plan de I'exptrience". 
la afermination de 1 applicabilite de la theorie ne serait que le constat de la realisation substantiejle dans le domane des dontes de certanes possibilitês prêvues dans le calcul de la theorie linguistique(6). Mais le fait que d'autres possibilites prévues par la theorie n'apparaissent pas attestees ou qu'aucune possibilite prévue pax la theorie le soit, n'infirmerait pas colle-ci. De mëme, les donnés qui attestent une des possibilités prevues par le calcul ne peuvent pas falsifier ou valider 1'apparej de definitions et des procedes de la theorie, car des donnés sont décrites de maniere "arbitraire" par celle-ci.si cette interpretation est correcte, la glossematique de Hjelmslev est a classer parmi les sciences formelles: elie se présente dans un cadre methodologique tel qu'il est impossible de dêcider si les definitions proposées par son appareil.conceptuel sont vraies ou fausses. Dans ce cadre d'ensemble, ou l'alternative formalisme vs. empixisme n'etait pas clairement tranchee, la notion de simplicite et sor utilisation en linguistique ajoutent encore a la confusion. Comme la plupart des points importants de la methodologie linguism tique, elle a merite en glossematique une attention particulière. Ulaall semble considerer la simplicite comme un desiaératum absolu, bien qu'il la subordonne à la coherence et à l'exhaustivité (131 p. 20 ) et Helmslev la pose comme element essentiel pour partager entre formulations concurrentes $(56 \mathrm{p} .18)$. La simplicits glossêmatique est nettement formelie: le recours à la simplicité est en derniere instance, dit Uldall (ibidem) un fait esthetique(7). formelle aussi la simplicite de Hockett, proposêe sous liêtiquette de "economie", bien que sa conception d'ensemble s'oriente vers une inguistique empirique (8).

Mais I'ambiguate formalismemempirisme, par rapport à la simplicitê apparast de maniêre particulierement nette dans les écrits de wajobson. Il insiste, a'une part, sur le fajt qu'une description en traits binaires est, parce que plus simple, plis adequate gu'nro autre, gui n'utilise pas des traits binaires; il s'agit par consequent d'une simpiicite formelle. 
Par ailleurs, ia esaib de justifier ja simplicite en fonction des processus psycho-physiologiques d'utilisation du langage; il incline ainsi vers une simplicite empirique(9).

Des déclarations très explicites de Noam Chomskv laissent penser yu'il a dêtinitivement ranché sur les problèmes posés par l2 simplicitë: d'une part, la simplicité, en grammaire gënérative, serait une simplicité empirique; par ailleurs, elle devrait etre interprétable psychologiquement par rapport a la capacité humaine d'acquisition du langage. Des textes clairs témoignent de ces prises de position.

D'abord, la simplicitê en granmajre gënërative est censêe être empirique; non seulement elle est posée comme telle mais, aussi, la simplicité formelie est. explicitement écartée:

"Encore une fois, nous disons de maniere emphatique que le choix d'une mesure de simplicité est un problème empixique Le problème de découvrir une mesure d'évaluation est très analogue à celui d'evaluer une constante en physique $[. . \vec{j}$. Il est aussi impossible de donner une justification A. PRIORI d'une mesure d'évaluation particulière qu'une valeur particulière à une constante de gravitution (Chomsky et Halle 3Ip.108).

"Il est un autre sens selon lequel on parle de simplicité, élégance, etc..des thêories (la physique de la relativité est plus élégante-simple- que la physique de Newton, l'astronomie de Copernic est plus simple que celle de ptolomeo, etc..P. Nous ne nions pas l'intêrêt de cette notion, mais nous n'avons rien a dire sur elle.c'est un concept qui n'appartient $n i$ a la Ifnguistique ni à aucune discipline Iinguistique particulière mais plutôt à I'épistémologie gênerale ou à la philosophie des sciences" (Chomsky et Halle 31 p.109-110).

Par ailleurs, la mesure de simplicité est une hypothèse associée à la capacite d'acquisition du langage: elle est par 
consughent, selon notxe teminologie, interpretable psychologiquement:

"Toutes ces thẹories ont, dès lors, besoin d'être complêté par une mesure d'Evaluation, si l'on veut rendre compte de

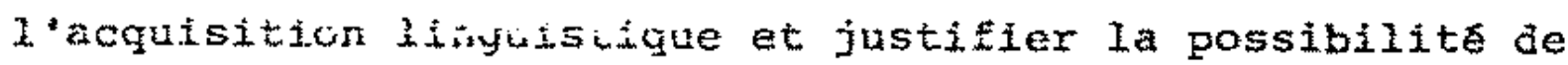
choisir des grammires particuliẽres; je continuerai d'adnettre provisoirement- comme je l'ai fait jusqu'a présent- que c'est là un fait empirique concernant la thêrie linguistique gênérale" (20b p.58/20 p.37). "Supposons en d'autres termes que nous considerions un modele d'acquisition linguistique comme un dispositif a' inpat-oxtiplt, qui determine à 1'output une grammaire generative particulière, étant donné a I'input certaines données Inguisticiues primaires; une mesure de simplicite, conjointe à une specification de (I)-(IV), constitue une hypothèse touchant la nature d'un tel dispositif. Le choix d'une mesure de simplicité est donc un problème empirique, ayant des conséquences empiriques" (20b p.59-60/20 p.38). "Un trait essentiel de notre théorie du langage c'est qu'eile comprend une mesure d'evaluation qui permet I'assignation de valeurs aux gramaires en concurrence.c'est sur la base de cette mesure d'evaluation qu'un enfant qui apprend une langue choisit une des gramaires (dont il y a en principe, une grande infinite) qui sont compatibles avec Le coxpus assez restrelnt de données linguistiques auquel il avait eté exposé. Ia grammaire qu'un enfant construit en apprenant sa langue native sera par consequent toujours celle quị est classée plus haut en termes de cette mesure d'evaluation" (32p.25I).

La lecture quelque peu attentive d'autres textes de granmaire génërative et I'observation de l'utilisation de la notion de simplicite dans la pratique montre que I'accord de ces declarations liminaires avec I'utilisation concreate de la notion de simplicitê ne s'est pas toujours réalisé. 


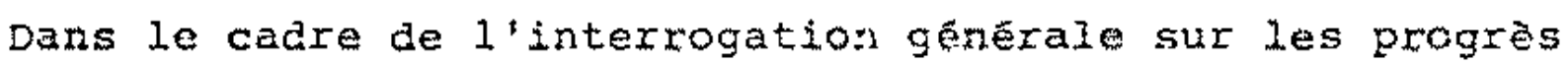
eventuellement accomplis par la gramaire gererative(10), nous nous proposons d'aborder le probleme de la simplicite en fonction des questions suivantes:

1.-Quelies sont la ou les notions de simplicité qui ont cours en gramalre generative? En particulier, la ou les simplicitês proposêes, sont-elies empixiques ou formelles? Sur quelies entités portent-elles?

2.-Ia ou les notions de simplicite empirique, sont-elles interprétables psychologiquement?

3.-Au-dele des deciarations de principe, est-ce que des hypothèses explicites de simplicité empirique et /ou de simplicite interpretable psychologiguement ont et formulees?Si oui, ontwelles ete validês ou infirmês?

4.-Est-ce que le traitement du probleme de la simplicite a ete avance par rapport a la situation anterieure, c'est a dire par rapport à Ia situation héritée par la gramaire genérative?

Les definitions adoptês ci-dessus de Eormel et d'empirique ne laissent pas la possibilite d'envisager une troisieme possibilité.celle-ci cependant dolt etre introduite pour pouvoir rendre inteligibles certains textes, mais elle portera non sur le rapport formulationmdonnees, mais sur le type a'entite consideree come donnê. En eftet, il est parfois impossibla, face a un texte, de dêterminer si une entits guej.concue peut jouer ou non le role de donnê par rapport a une proposition determines; dans ce cas, on parlera de propositions eventuellonent empirigues. Nous disons qu'une proposition len particuliex, une formuation de simplicite) est eventueliement empixique, si, acceptant le caractere de donne d'une entits quelconque dont le statut est douteux, la proposition en question etait empirique. 
Désormais, pour faire reférence à la simplicité, on utili-. sexa MS (mesure de slmplicite) et trois fomes verbales:difing: caracteriser, a l'indicatil et caracteriser, au conditionnel. La première sera utiliše dans le cadre dune simplicité fommelle, la deuxieme, dans celui a'une simplicite empixique et la troisieme, pour les cas d'une simplicité eventueldement empirique. La formule"MS asfintt A comme plus simple que B", indique qu'aucune condition de verification externe ne sera exigee de l'entité $A$, selectionne come plus simple; la formule"Ms. caracterise A comme plus simple que B", indique que 1'entite A, sélectionne comme plus simple, devra etre mise en rapport avec des donnêts susceptibles de tester l'hypothese sur la simplicite; la formule "MS caxactêriserait A come plus simple que B" sera rêservée pour les cas douteux, Eventuellement empiriques, ol l'entite $A$ est mise en rapport aveo des donnees externes, mas dont $1 e$ statut est confus, le tat de savoir si ces donnees sont susceptibles ou non de tester une hypothèse n'etant pas clatr.Les notions de définix et de caracteriser (a l'indicatif et au conditionnel) peuvent s'appliquer aussi bien en termes absolus qu"en termes graduels, c'est à are qu'une entit A quelconque peut tre soit pius simple que B, soit plus simple que $B$ à un degre determine; on convient donc que les termes utilises peuvent recevoir les deux interpretations.

L'entite $A_{\text {, }}$ dans le cadre de la problëmatique ici aboraée, est une entité proposte en linguistique, tués géneralement une grammaire ou une ou plusieurs règles d'ume grammire. On utilusera par conséquent grammaire aussi bien pour faire référence a une grammatre universelie ou particuliesre complete qu'à un sous-ensemble d'une ou de plusleurs regles ou entités d"une gramaire on note gramaire universelie par gu et gramaire particuliere par GP. On dira qu'une GP est determinee par une GU si GP respecte los restrictions et le schema genéral de l'organisation désinds pax Gu.par representation nous comprenons cet objet theorique note symboliquement et ai est destine a rendre compte dun enoncé: les arbres ou parenthétisations êtiquetés constituent tyojquement des representations.Une gramajte specifie fou 
engendre) un ensemble infini de représentations.Les donnéessauf indication contraire- sont constituées par les énoncés d'une part, et d'autre part, par les jugements metalinguistiques intuitifs portés sur eux (d'ambiguité, de gramaticalitê, de paraphrase, etc...); on les placera sous la rubrique langage. Nous supposons qu'existe la possibilite de determiner l'identité entre grammaires (particulięres ou universelles), entre reprësentations et entre donnees. En fait, en ce qui concerne les objets theoriques (gramaires et representations), le presupposé sur.la possibilité ou non de les identifier va au-dela des possibilités actuelles de la théorle linguistique:une partie de la discussion entre sous-écoles rivales est centrēe sur le problème de déterminer les identités ou différences (substantielie, notationnelle. etc..), entre hypothèses alternatives(11).

Pour notre discussion ce point est relativemant secondaire car nous utiliserons les identifications des entités rapprochēes qui ont eté explicitement ou implicitement faites par celui qui propose une MS.

Nous acceptons également qu'existe la possibilité de déterminer l'équivalence entre grammaires particulières et entre gramaires:airvepselles.on dira que deux GP sont entièrement équivalentes, si elles-mêmes et les representations qu'elles specifient sont differentes et $s i$ ces représentations peuvent rendre compte, dans les mêmes conditions d'adequation externe relatives a thaque enonce et aux jugements metalinguistiques portes sur lui, de tout langage appartenant a la langue particuliere en question.Par ailleurs, on dira que deux GP sont equivalentes par rapport a un langage $x$, si elles-memes et les representations qu'elles specifient sont differentes et si ces representations peuvent rendre compte d'un même langage $x$, appartenant a la langue particuliere en question, dans les mêmes conditions d'adequation externe relatives à shaque énoncé et aux jugements métilinguistiques portés sur Iui.etre entièrement équivalent et etre equivalent par rapport a un langage $x$, ne sont donc pas la même notion: si deux GP qui sont entièrement Equivalentes doivent être équivaientes par rapport a un langage $x$, le contraire n'est pas vrai.Dans les formulations proposées par la 
suite, on trouvera deux situations d'équivalence entre GP: (a) les GP considêxées sont soit entièrement équivalentes, soit Equivalentes par rapport ã un langage $x$; ce sont les cas oí les les textes d'où les formulations sont tirés exigent une equivalence entre les GP mais ils ne permettent pas de trancher entre le type d'equivalence exige pour que la so soit susceptible d'être appliquée (formulations I,III,IV,etV); on peut penser que dans tous ces cas, on souhaite appliquer la Ms a des GP entièrement Equivalentes, bien que des illustrations re portent que sur des GP équivalentes par rapport à un langage déterminé; (b) les GP considerées ne sont pas entièrement équivalentes, mais équivalentes par rapport à un langage determiné (formulations VII et VIII).

I'équivalence au niveau des gu doit se définir par le biais de l'équivalence des GP qu'elles determinent.plusieurs cas sont à prêvoir.tant donné deux GU, chacune de ces GU peut dêteminer, pour toute langue particulière, une GP qui est soit entièrement equivalente à la GP determinée par i'autre Gu, soit équivalente fax rapport à un langage x.Ou bien, les Go peuvent déterminer, pour certaines langues naturelies, des GP entièrement equivalentes et, pour d'autres langues naturelles, des GP équivalentes par rapport a un langage x.Dans les formulations qui suivent, aans un seul cas (formulation II) il a eté possible de dégager une situation d'équivalence claire entre deux GU: ce sont des GU qui, chacune, determine, pour toute Iangre particulière, une GP qui est entièrement équivalente à la GP déterminée par l'autre Gu. on dira, dans ce cas, que les Gu sont entièrement équivalentes, et on s'abstiendra de proposer une terminologie pour les autres cas possibles d'équivalence entre Gu.

Au moyen de lettres capitales on distinguera des grammaires universelles différentes:GU-A et GU-B sont des gramaires universelles différentes. En revanche, on utilisera les numéros pour distinguer les grammaires particulières et les langages: GP-1 et GP-2, Lang-I et Lang-2 indiquent, respectivement, des gramaires particulières et des langages différents. 
II n'est pas toujours aisé de discuter les ecrits de la grammalre gênerative, un certain type de vocabulaire- celui, en particulier, qui porte sur les notions gênérales- etant souvent employe sans tigueur excessive; conme; par ailieurs,il est imposstble de aiscuter un texte peu explicite ou ambigu, 11 a ete nécessaire de reformuler les textes originaux en propositions censees etre claires et susceptibles d'etre discutés.te risque de deformation de la pensee ortginale est certain; pour le xéuire autant que possible, chaque reformulation est suivie des textes originaux qui ont permis la reformulation et/ou des réfertences bibliographigues précises.

Au 5 2, qui suit, seront groupéss les formulations qui comportent une simplicite formelie et dont la presentation originale n'était pas liée a la notion de gênéraisation.te \$ 3 sera consacré aux simplicites mises en rapport avec la notion de generalisation; on $y$ explicitera des simplicités formelles et des simplicites eventuellement empiriques.te $\$ 4$ prësentera les formulations comportant une simplicite empixique. Au 6 or discutera la possibilite d'interpretation psychologique de la notion de simplicite et au $\$ 6$ on tirera le bilan d'ensemble, par rapport aux questions postes aans ce premier paragraphe et

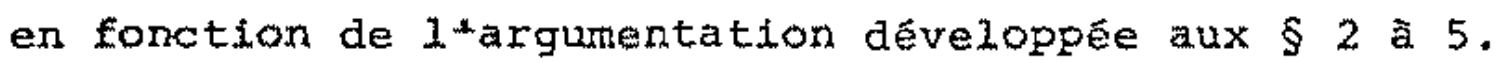

\section{Simplicités formelles.}

Il est possible de cerner au moins trois Ms formelles qui different entre elies en fonction des entites sur lesquelies porte la comparatson et/ou des consequences que chaque MS entraine pour la thcorie linguistique.particuliérement importantes sont les formulations du $\$ 2-1$ et du $\$ 2-2$, car les Ms qui. $y$ sont prësentées sont directement impliquêes dans le problème delicat du choix entre Gu concurrentes.

II-1 Formulation I

Soit GU-A et GU-B; qui determinent, respectivement, GP-1 et: GP-2, GP-1 et GP-2 sont soit entierement equivalentes, soit 
equivalentes par rapport a un tang-1.

MS asfinit GP-1 come plus simple que GP-2.

pax conseguent, GU-A dolt etre préferée à GU-B.

L'essentiel de la formulation prêcédente est I'utilisation de la MS poux choisir entre Gu concurrentes: on doit preferer celle des Gu qui determine la GP plus simple susceptible de renate compte d'un langage donne. cette formulation se degage, pax exemple du texte suivant:

"La preuve la plus decisive de l'inadequation a'une theorie linguistique consiste a montrer qu'elle ne peut absolument pas s'appliquex a une langue naturelie. Une preuve plus falble, mais parfaitement suffisante, de son inadequation, serait de montrer que la theorie ne s'appligue gu'avec lourdeur, c'est a dire que toute grammaire construite selon cette theorie est fort complexe, forgêe ad hoc, et non revelatrice, que certatins procédés trés simples de description des phrases grammaticales ne peuvent s'accomodex des formes de la gramnaire qui leux correspondent, et que certaines propriétes formalies fondamentales des langues naturelles ne peuvent etre utilisées pour simplifier les granmaires 4.7.

La seule façon de tester 1'adequation de notre apparej. est a'essayer de i'appliquer directement a la description des phrases anglaises. Des que nous consiaerons une phrase un peu complexe et, en particulier, Lorsque nous tentons de definir un ordre entre les règles gui produisent ces phrases, nous nous heurtons à de nombreuses dificultes 6 ...Jje me bornerai a esquisser quelque cas stmpies ol des progres considerabies peuvent etre realisés sur les gramaires de la forme $(29 \mathrm{~b} p \cdot 39-40 / 29 \mathrm{p} \cdot 34-35)$.

Le passage qui precede annonce les grands traits d'un raisonnentent qui va se concretiser tout au long du chaptre v 
des Structures Syntaxiques et qui reapparat aussi ailieurs: la GU entièrement composee de règles syntagmatiques est inadequate parce que les GP qu'elle determine- presentée sous forme de regles de I'anglais- sont compliquees; par contre une Gu qui incorpore des transformations est adequate parce qu'elle determine des GP qui sont susceptibles de rendre compte des mêmes données et qui sont plus simples.A la suite de la discusston de plusieurs cas concrets, la conclusion d'ensemble qui en est tirêe s'integre, elle auss1, dans cette formulation I:

"Nous venons de voir qu'une grande varieté de phénomènes apparement distincts se mettent en place tout naturellement lorsque nous adoptons le point de vue de l'analyse transformationnelle et que, par conséquent, la gramaire de l'anglais devient beaucoup plus simple et ordonne.c'est la l'exigence premiere que toute conception de la structure inguistique (c'est a dare tout modale pour la forme des grammatres) doit satisfaire.Je pense que ces considerations justifient amplement l'affirmation enoncée plus haut, a savolx que les conceptions syntagmatiques sont fondamentalement inadéquates et que la theorie de la structure linguistique doit être dêveloppée dans le sens du présent exposè de l'analyse transformationnelle" (29b p.75/29 p.68-69').

Le schema de raisonnement qui preside à la FormulationI reapparait souvent dans les écrits de grammaire génêrative. Dans 20b p.127/20 p.87, Chomsky affirme que le lexique doit être separe des règles syntagmatıques parce que "la grammaire peut etre simplifiée de façon importante si [les propritéts idiosyncratiques des formants] sont exclues des rêgles de récriture et enregistrees dans les entrees lexicales ol elles vont a leur place la plus naturelle". Et il ajoute par la suite: chaque fois gue L'on essaiera de construire une grammaire précise, i.l se rëvelera rapidement que bien des formants ont des caractéxistiques grammaticales unigues, ou presque, de sorte que la simplification de la gramaire qui peut etre ainsi rélisée seca certainement substantielle". 
On compare donc GP-1 et GP-2 que l'on suppose rendre compte des mêmes donnêes et qui sont respectivement déterminées par GU-A et par GU-B. GU-A est une grammaire universelle avec lexique;GU-B sous-catégorise les formants dans les règles syntagmatiques et ne possède pas de lexique. Puisque GP-1 est plus simple, la solution proposée dans le cadre de GU-A est adoptêe sur un plan général.on constate donc qu'une solution dans le domaine de la GU est choisie en fonction dune simplicité formelle portant sur les GP (12).

Le mëme raisonnement apparart applique au domaine des faits phoniques. Par exemple, Halle (피 p.91) choisit GU-A(avec règle's ordonnês) au detriment de GU-B (avec règles non ordonnées) de la manjère suivante.GP-1 et GP-2, determinês respectivement par GU-A et GU-B, sont présentées comme étant toutes deux susceptibles de rendre compte de la formation du pluriel en anglais.Halle observe que "les longueurs relatives des deux ensembles de propositions ( c'est à dire, des règles non formalisẹ̈s) reflètent leur simplicité relative.par consequent, il est obligatoire d'introduixe l'ordre dans cette circonstance si nous désirons satisfaire notre critère de simplicité". Le résultat en est qu'on choisit GU-A, GP-l ayant ete definie comme plus simple que GP-2 (13).

II-2 Formulation II.

Soit GU-A et GU-B, gut sont entièrement équivalentes. MS définit GU-A comme étant plus simple que GU-B. Par consëquent, GU-A doit etre préferèe à GU-B.

La simplicité formelle porte cette fois non sur wes GP mais sur les GU qui les dêterminent. 'La présentation la plus claire en est faite par postal (14).

La particularité qui apparait explicitement dans la formulation de postal est le recours a des operations ensemblistes:posseder moins d'éléments est concrétisé comme A etant inclus dans $B$, le complément de A dans $B$ n'etant pas vide; cela implique que les eléments $x$ sur lesquels porte la quantification de Ms sont des eléments qualitativement différents: 
"Je ne voudrais me presenter comne un expert en philosophie de la science ni suggerer que les querelles theoriques en linguistique sont susceptibles en general de trouver leux solution dans la philosophie de la science.Cependant certains points sont relativement clairs.Deux théories differentes du même domaine etant données, il est possible de faire un choix clair entre elles si certaines relations logiques sont valables entre ces theories.En particulier, $5 i$ l'outillage théorique ("theoretical machinery") d' une theorie est inclus dans celui de la deuxième et si celui-ci possède, en plus, un certain outillage thêorique adaitionnel, alors toutes. les autres choses etant identiques, la : première theorie, conceptuellement plus restreinte, doit etre choisie" (107 p.135).

Les formulations I et II qui précèdent proposent exactement le contraire de ce qui est nié avec force par ailleurs; non seulement la simplicitê formelle est rejeté (cf.ci-dessus) mais explicitement on aftizme que la MS utilisée dans le cadre de la grammaire générative ne doit s'employer pour comparer ni des GU différentes ni des GP déterminêes par des Gu différentes:

"Il apparait egalement que des mesures d'évaluation comme celles dont on a discuté dans la littérature consacrée à la gramaire generative ne peuvent etre employées pour comparex différentes theories de la grammaire; il est complètement absurde de comparex a l'alde d'une telle mesure une grammaire appartenant a une classe de gramaixes proposées avec une Gramaire appartenant à une autre classe $6 . .7 C^{\prime}$ est evidement une question fondamentale que ce dernier pro: blème et 1 ton devrait également la rëgler en se fondant, dans la mesure du possible, sur des bases empiriques d'adéguation descriptive et explicative.Mais ce n'est pas la question qui est mise en jeu lorsqu"on emploie une mesure d'evaluation pour tenter d'attejnare 1'adéquation 
explicative.A titre d'illustration concrete, examinons si les règles d'une grammaire dotvent être non ordonnées (appelons cela la theorie linguistique $\mathrm{Tn}$ ) ou ordonnees d'une manière specifiqus (théorie To). A priori, on ne connalt aucun sens absolu de la "simplicite" ou de "l'elégance", développes dans la thêrie linguistique cu dans 1 'epistémologle générale, qui permette de comparer in et To. Il est donc absurde de soutenir qu"en un sens"absolu" Tn soit "plus simple" que To ou inversement.Il est aisé d'inventer un concept génếral de "simplicité" qui donnera l'avantage à Tn sur To ou bien à To sur Tn; ce concept n'aura aucune justification connue, dans aucun de ces deux $\operatorname{cas}^{\prime \prime}(\underline{20 b} \mathrm{~g} \cdot 60 / \underline{20 p} \cdot 38-39)$.

II-3 Formulation III.

Soit $G U-A$, gui determine $G P-1$ et $G P-2$. $\mathrm{GP}-1$ et $\mathrm{GP}-2$ sont soit entièrement equivalentes soit équivalentes par rapport à un Lang-1. MS definit GP-I comme plus simple que $\mathrm{GP}-2$. Par conséquent, $G P-1$ doit être préférée à GP-2.

Cette formulation-ci, a la différence des précédentes, ne s'applique qu'a des GP déterminêes par une même GU; elle résume les textes qui suivent, empruntes aux structures syntaxigues:

"Une exigence encore plus modeste serait qu'etant donné un corpus et deux grammaires $G 1$ et, $G 2$, la theorie dise laquelle des deux est la mellleure grammare de la langue d'oì le corpus est extrait. Dans ce ças nous pourrions dire que la théorie nous fournit une procédure d'evaluation des grammaires" (29b p.57/29 P.51).

"Nous pensons qu'il serait dêraisonnable d'attenàre d'une theorie linguistique qu'elle fournisse pius qu'une procedure pratique d'evaluation des grammares" (29b p.58/29 p.52) "Dans chaque cas, notre seul souci a eté de determiner la complexité de la grammaire et nous avons cherche à montrer 
que l'analyse proposée etait manifestement plus simple que les autres analyses possibles que nous avons rejetés" $(296$ p.92/29 p.83).

Ce n'est pas seviement dans les structures syntaxigues que cette formuletion est proposee (15). Dans Aspects.... certaines ues solutions partielles proposées au problëme de l'insertion lexicale sont fondées sur le même type d'argumentation.par exemple le fait que le verbe et 1 'adjectif possèdent des traits de selec-: tion contextuels qui sont determines par les traits de sélection du nom est justifié en fonction d'une Ms encadré dans la FormuIation IIT:

"Br̀ef, en décidant que l'analyse des Verbes en symboles complexes fera liobjet d'un choix indépendant et que les Noms seront selectionnés sulvant une règle fondée sur les Verbes, on est amene a compliquer considexablement la grammaire.Les problemes sont multiplies quand on tient compte des règles sélectionnelles indépendantes Nom-Adjectif. En raisonnant d'une maniere analogue, nous pouvons exclure Ia possibilité que le Sujet choisisse le verbe et le verbe 1 'Objet" (20b P.160/ 20 p.115).

Peu de remarques doivent être faites sur les formulations I a II.Dans les trois cas il s'agit d'une simplicite formelle qui conduit aux difficultes difficilement surmontables de toute simplicite formelle (cf.notes 7 et 9). Celui qui a propose une hypotinese a une:tendance marquée a la considerer comme étant plus simple et plus belle que l'hypothèse proposée par son collègue ou son concurrent.par ailleurs, pour pouvoir appliquer les formulations avec un minimum, de rigueur, il serait indispensable, dans les cas des formulations. I et II, de resoudre au préalable le problème de la comparaison des theories qui, parce que différentes, utilisent des entités differentes bien que la même notation graphique soit utilisée.Est-ce qu'une entité "sN", d'une grammaire syntagmatjque peut etre comptëe comme une entité "SN" d'une grammaire transformationnelle? Pour ne pas glisser tans l'erreur de quantifler des entités dont la caracterisation est différente, il faudrait"traduire" le système notationnel de chaque théorie à 
une sorte de sur-notation commune et appliquer la us sux le résultat de telles trađuctions.Dans l'êtat actuel des connaissances sur l'aspect formel des theories linguistigues, il est pratiquement impossible d'envisager cette possibilité.

La Eormulation I apparast, parmi celles qui ont deja ete citêes, comme celle: qui a laissé le plus de traces dans le développement de la thêtorie.Dans un certain sens cette formulation dérive du principe génertal de simplicite, souvent invoque pour cholir entre thëories concurrentes (cf.note 4). La simplicite a pour elle une certaine tradition d'elégance, de beauté classique, d'harmonie, etc...;il se peut qu'un jour on puisse expliquer l'origine psychique de ce sentiment (cf.ci-dessous $\$ 6$ ). Mais la Formulation I ne se laisse pas entierement englober dans ce principe general.Ce qui est propre a la Formulation I, c'est que la MS ne s'applique qu'aux GP et non aux GU respectives, malgre le fait que la fonction de cette MS est de selectionner la GU. C'est a dire qu"une simplicité formelle sëlectionne finalement la GU mais de manière mediate, par le-biaid de son application aux GP. Nous ne reconnaissons aucun principe dans la methodologie génerale des sciences d"où on puisse déduire que la simpilcite doit portex sur quelques-unes des hypothèses ae deux ensembles d'hypothèses rendant compte d'un domaine dëtermine et non sur ces ensembles considerts dans leur totalite.par ailleurs, 11 faut observer que si l'on utilise la même MS intuitive qui permet de cholsir entre GP dEterminées par GU aifférentes, le resultat des cholx sur le plan des GU est l'opposé de sur celui des GP.Il est vraj que souvent, dans les exemples où la formulation $I$ a ete appliquee, il $Y$ a des cas ou effectivement "on a come le sentiment" qu'une GP-1 dêterminée par GU-A'est plus simple que GP-2 déterminée par Gu-B.Mais le mème"sentiment" réapparait, en sens inverse, si $I$ 'on rapproche $G U-A$ et $G U-B . G U-A$ avec règles syntagmatiques et transformations apparait comme moins simple que GU-B avec les seules règles syntagmatiques.Et il est impossible, sur un plan formel, de justifier cette prêference pour la simplicité d'un certain type d'hypothèses au dêtriment de la complexité des hypothèses d'un autre type (16). 
La notion de genéralisation est particulièrement importante en gramaire generative. Utilisée pour justifier le choix d'nypotièses concurrentes, en particulier, gxamualres universelies, elle n'a pas cependant été dêfinie avec precision.Certes, on entend par generalisation le fait d'englober dans une proposition plus generale le contenu de plusieurs propositions particulières, qui deviennent ainsi derivables de la proposition plus générale. Mais cette idee intuitive est rebelle à une formulation plus précise; en particuliex, on s'efforce de degager les traits susceptibles de distinguer une généralisation significative a'une generalisation qui ne lest pas (17).

II ne rentre pas dans le cadre de cette discussion de prêlser la notion de gêneralisation. On ne l'abordera donc qu'en ce qui concerne ses rapports avec la notion de simplicité.sans poser le problème de savoir ce qu'est une gênéralisation (celles dont on fera état sont censées être "significatives") nous envisageons deux possibilites:

1) Les gênéralisations sont des données; elles sont par consequent susceptibles de tester une hypothèse;

2) Les generalisations sont le résultat dune definition; elles appartiennent à l'appareil conceptuel d'une theorie et ne sont pas susceptibies de tester une hypothase.

Vu leur statut douteux, il est impossible, meme dans le cadre de la posstbilite (1), d'accepter les generalisations comme des donnees à part entiere; pour cette raison, on utilisera caractériserait poux les MS censées être testées par rapport a des generalisations. III-I Formulation IV

Soit $\mathrm{GU}-\mathrm{A}$, qui determine $\mathrm{GP}-1$ et $\mathrm{GP}-2$.

GP-1 et GP-2 sont soit entlèrement equivalentes, soit equivalentes par rapport a un lang-1.

MS definit GP-1 comme plus simple que GP-2.

Par conséquent, GP-1 possede une genêralisation.

Formulation $V$

Solt GU-A, qui determine GE-I et GP-2. 
GP-1 et GP-2 sont soit entierement equivalentes, soit equivalentes par rapport a un Lang-1.

GP-1 possède une gêneralisation, ce qui n'est pas le cas de GP-2. MS caractêlserait GP-1 comme pIus simple que GP-2.

Par conséguent, MS serait validée.

S1 NS n'etait pas susceptible de caracteriser GP-1 comme plus simple que GP-2, MS seratt infirnee et devrait être modifié.

Ces deux formulations sont nettement differentes, l'une comportant une MS formelle, I'autre une MS éventuellement empirique.or, malgré cette difference fondamentale, on doit les degager l'une et l'autre, au moins partiellement, d'un neme texte gui, par conséquent, est ambigu; le volot divisé en sous-textes par des lettres intercalees $(b)$ (b)); groupes avec d'autres citations sur la même question, ils permettront, par la suite, de faire des citations plus analytiques:

a) "Le trait systematique utilisê toujours, ouvertement ou nor, pour choisir parmi des desexiptions alternatives, c'est le degré de généralisation obtenu.on a une généraisistion loxsque des assertions alfferentes a propos a"eléments diffërents peuvent être remplacés par les menes assertions ou par des assertions similaires. Il est par conséquent naturel, et 11 n'est pas difficile, de dêvelopper des systèmes de notation qui, convertissant les considerations de complexite en consldérations de longueur, conduisent a une definition partielle de complexite" (Chormsky 30 p.242).

b) "Dans 1'etablissement d'une mesure d'evaluation des grammaires, le problêne principal est de detexminer parmi les generalisations concernant une langue, celles qui ont une portee ("which generalizations about a language are significant ones") et 11 faut choisir la mesure d'évaluation ae façon a conner l'avantage a ces dernistes". 
c)"Nous avons hie generalisation lorsuran ensemble de regles concernant des elements distincts peut être remplacé par une rềle unique (ou, plas gênéralement, par des regles partieliement identiques) concernant l'ensemble tout entier, au encore lorsqu'il est possible de montrer qu'une "classe naturelle" a'éléments passe par un certain processtis ou un ensemble de processus analogues".

d) "ninsi le choix d'une mesure d'évaluation constitue une dêcision sur la nature des "processus analogues" et des "classes naturelles"-en bref, sur la nature des generalisations importantes ("In short, what are signiflcant generalizations")".

e) "Le probleme est de construire une procédure assignant ¿ une rrammaire une me.jure numbrique de valeur sur la base du degré de gênéralisation linguistique significative auquel elle parvient".

f) "La mesure numerique evidente que I'on peut appliquex a une gramalre est la longueur, definie sur la base du nombre de symboles.Mais si ce doit etre une mesure douée de sens, 11 est necessaire de construire les notations et de restreindre la farme des règles de telle façon que les considerations importantes de complexite et de generalite soient converties en considérations de longueur- cela afin que les géneralisations rêlles abrègent la gramaire et que les pseudo-géneralisations ne le fassent pas.Ce sont ainsi les conventions de notation adoptees pour pressenter une grammaire qui dêfinissent la"generalisation importante" "significant generalization"), si l'on admet que la mesure a'évaluation ast la longueur" (Chomsky 20b p.63-64/20 p.42). 
g) "Il (le systeme de notation) a ete propose comme une explication de la notion "generalisation linguistique significative"; le degre de gêneralisation linguistique signiflcative atteint par une gramaire-sa"sımplicité", dans le sens technique du terme- est mesure par le nombre de symboles qui apparaissent dans le schéma sous-jacent qui se développe en cette gramaire par l'utilisation du système de notation" (Chomsky et Halle 32 g.36).

Dans la Formulation IV, la MS est utilisêe pour definir, dans le sens formel, l'existence d'une gẹneralisation.Les textes qui permettent cette interpretation sont a in fine, $\underline{c}$ et, en particulier, 9 , in tine, et g.Nous comprenons que GP-1 possede une géneralisation, à la difference de $G P-2$, qui $n$ 'en a pas, si GP-1 est plus simple que GP-2: plusieurs regles de GP-2 sont remplacêes par une seule rêgle de GP-1.Selon cette interprétation, "posséder une GP une généralisation"est le résultat d"une defini-. tion(18). La Formulation IV "complete" en quelque sorte la Formulation III; la différence en est que, d'apress la cormulation IV, la GP-I qui est définie comme étant plus simplè est, par làmême definie comre posseant une generalisation, La MS de la formulation IV est posẹe comme un principe gênêral, valable non pas pour quelques règles mais pour toute:la gramaire, par Jacobs et Rosembalum (19):

"Par exemple, une grammaire où on a doublé une certaine quantite d'information pour dẹcrire un phénonène particuiler est moins générale qu'une grammaire pour lequêne phénomène où cette information est présentête une seule fois" (63 p.224).

La Formulation $V$ se dégage des textes $\underline{b}$ et $e$ ci-dessus présentês.A la différence de la Formulation IV, la Formulation $V$ serait empirique si la generralisation était une donnée a part entiere.en effet, 1 a lecture stricte de ces textes induit a interprêter que la MS devrait etre modifiée jusqu'a ce qu'elle soit capable de s'adapter aux gênéxalisations rencontrés, dans ce 
sens que si une GP possedalt une generalisation, elle devraft être sanctionne comme plus simple; si elle ne l'etait pas, ce serait la MS qu'il faudrait corriger.C'est a cause de ce schera susceptible de tester une us par rapport a une généralisation que celle-ci serait trentuellement empirique.

ta Formulation $\vee$ a etê utilisê d"une manière plutot marginale(20). En revanche, la formulation qui suit, dans le 5 III-2, apparemnent proche de la $v$, majs ed fait très aifférente a ete employée en gramaire genérative de maniżre non accidentel1e.

III-2 Formulation VI

Soit GU-A, gui determine GP-I et GP-2.

GP-1 rerd campte du Lang-1 et GP-2 du Lang-2.

GP-I rena conpte des donees telles gr'elle doit posséfer une generalisation, ce qui n'est pas le cas potr gP-2.

MIS definit GP-1 conme plus simple gue Go-2.

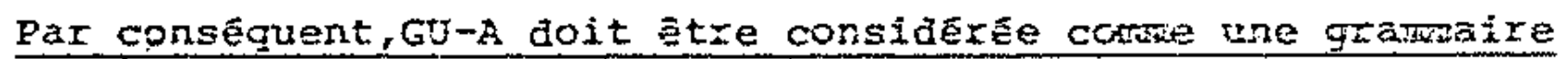
universelie susceptible d'expriner une gersazisetion.

Si ks n'etait pas susceptible de definir GP-I comie plus sirolo. gue $G P-2, G U-A$ devrait etre modifiee.

Dans cette Formulation VI, come dans la prececiente Eormulation $V$, la MS est mise en rapport aỵec des GP dont 1"une devrait possêder une gênéralisation et 1.'zutre ron, etant 1 'une et l'autre determinées par une nême Gu.Mais I analogie extre les dewx formulations s'arrete ici.En effet, dans la Formulaton VImais non alans la $V-G P-1$ et GP-2 rendent compte des dombes differentes.par ailleurs, dans la Formulion VI, ce n'est pas la kS qui devrait etre mise en cause si elle n'était pas susceptible de définir come plus simple GP-1, censée posseder une généralisation, mais $1 \mathrm{a}$ GU qui a determine GP-1 -De manière analogue, si la kis defintssatt Gp-h coware plus sirple, on ne devrait pas pour autant en conclure gue Ia ks serait validé; dans ce cas, ce serait la Gu qui a determine GF-I gui le serait. Ies exenples qui suivent, empruatés a chonsky et halle 132 p. 00 et suivantesl permettent d'illustrex cette formalation et ses conséquences (cf.egalement Ie texte (f) de la cttation prectedente empruntè à chomsky 206 p.64/20 P.427. 
Soient les paires de.règles qui sulvent:

$\begin{array}{ll}(a-1) & i \longrightarrow u \\ (a-2) & i \longrightarrow j\end{array}$

(b -1$)$

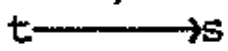

$(b-2)$

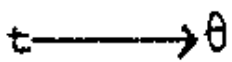

$(c-1)$

(c -2 )

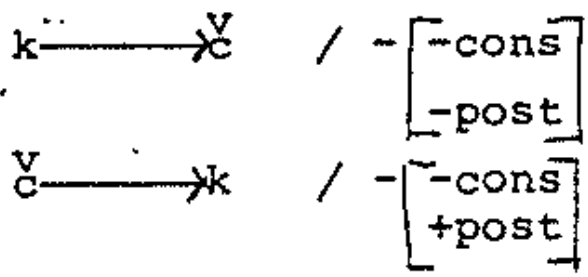

Chomsky et Halle affirment que dans ce cas (et d'autres analogues qui y sont présentés) l'exemple 1 comporte plus de traits distinctifs ou le même nombre de traits distinctifs que 1 'exemple 2 correspondant bien que, dans chaque paire, l'exemple 1 soit "plus attendu dans une grammaire que le cas (ii) (= exemple 2) et devrait par conséquent etre "plus simple" en termes d'une mesure significative empicique d'evaluation"(32 p.401).Et plus loin ils ajoutent:

"Tous ces exemples, et beaucoup d'autres, marquent la nëcessitê d'êlargir la thếorie pour intégrer les effets du contenu intrinsèque des traits et pour distinguer les cas "attendus" ou "naturels" des règles et des contigurations de symboles des autres qui sont non attendus et non naturels.Dans le sens linguistiquenent significatif de la notion "complexite", une rêgle qui sonorise les voyelles ne devrait pas contribuer à la complexité de la grammaire mais une rejgle qui les assourdit devrait le faire, tandis que dans le cas des obstruantes la décision opposée serait requise". (32 p.402).

Dans la suite de leur raisonnement, les consequences tirêes des cas précédents sont claires: la GU universelle est modifiée et on $y$ introduit la notion de marque afin qus, zars les cas necessaires, les GP qu'elle determine soient definies comne plus simples. 
Cette maniere de rasonaer implique gu'on dispose d'une rS "Evidente", qui n'est plus consideree comme hypothese empirique, l'interrogation ne portant pas sur elle mais sur la Gu.C'est effectivement $l^{\prime}$ idee que l'on trouve explicitée dans d'autres textes:
"La mesure numerique evidente qué l'on peut appliquer à une grammaire est la longueur, definie sur la base du nombre de symboles" (Chomsky $20 \mathrm{~b}$ p.64/ 20 p.42).
"Probablement il est mieux de considerer une mesure d'eva- luation comme un procédé pour assigner un entier à une grammaire, un entier plus petit correspondant a une valeur plus haute" (Chomsky et Halle 31 p.108).
"I a "valeur" d'une suite de règles est la réciproque du nombre de symboles dans la representation minimale" (Chomsky et Halle 32 p. 334).

Le schéma de raisonnement qui se dégage, schéma extrēmenent important, car il est à la base de la justification de la grammaire universelle, rest donc le suivant:

1) Deux GP (GP-1 et GP-2) sont donnēes; elles rendent compte des données diffêrentes mals sont détexminêes par la même GU-A.

2) On saitqu'une de ces grammaires doit posséder une généralisation( ou, avec une terminologie encore plus métaphorique, gu'elle est plus "naturelle" ou" attendue"), ce qui n'est pas le cas pour l'autre.

3) On đispose a'une MS postulēe et admise.

4) La GU qui dêtermine GP-1 et GP-2 est une GU adéquateou, en d'autres termes, est une GU susceptible d'exprimer une gênéralisation- si la GP censée posseder une généralisation est définie comne plus simple par la MS; dans le cas contraire, GU n'est pas adequate et elle doit être modifiée.

Ce schema de raisonnement a également été utilisé dans le domaine syntaxique, par exemple dans Aspects (p.128-134/p.90-95) à nropos des règles de sous-catégorisation.La Formulation VI y est concretement utilisee de la manière suivante (les numéros entre parenthèses, sauf indication contraire, correspondant aux numéros utilises dans Aspects pour individualiser les règles ou les 
conventions):

$\mathrm{GP}-1$ : Règle (34)
$\mathrm{GP}-2$ : Règle (35)
GU-A : Convention de notation des traits de sous-catégorisation.Les traits de sous-categorisation sont explicités et a droite de la rẹgle qui operre sur $(+V)$ et dans les inaications du contexte de $(+v)$.

MS : ĩon entièrement explicitée mais fondée sur le nombre de symboles utilisés dans $G P$.

on sait : GP-I doit posséder une généralisation;qui est absente de GP-2.

on constate:par application de MS, GP-I n'est pas définie comme plus simple que GP-2.

Conclusion: GU-A doit être modifiée.

Le même texte présente la Formulation VI utilisée en sens contraire, c'est a dire, dans le sens de la confirmation de la GU: GP-l : Règles $(40)$ et (42); la règle (42) est equivalente à la rè-. gie (34) de l'exemple précedent. GP-2: Règle (40-bis) (non explicitée dans le texte; devrait être comparée à la règle (40)) et régle (35), comparée a (42). GU-B:Convention de notation des traits de sous-catégorisation (convention (36)). Les traits ne sont explicités que dans les indications de contexte de la règle qui opère sur $(+V)$. MS: Non entièrement explicitée mais fondée sur le nombre de symboles utilisés dans GP et identique a la MS de la règle précédente.

On sait:GP-l doit possêder une généralisation, qui est absente de GP-2.

on constate: par application de $M S, G P-1$ est définie comme plus simple que GP-2.

Conclusion:GU-B est adécquate ou, plus exactement, plus adéquate que $G U-A(21)$.

La wS de la formulation VI est formelle(22). Elle l'est ron pas parce que les notions de "posseder une genéralisation"ou "être naturel", etc., qui doivent qualifier les gramaires particulières sont peu sūres, non objectives, etc. Le statut douteux de 
ces notions ne dyrat permettre que de classer la Ms come eventuellement empixique Mais la MS de la Formulation VI est fortelle parce gu'elze ne peut être testee par aucune donne; meme si les notions qui qualifient les gramaires particuliêres accedaisnt, dans aes formulations ultéleures, a la catégorie de donnés, 1a MS continuexalt a être formelle.Dans le schema de raisonnement ol elle prend place, la MS est acceptée comme un a priori qui n'est pas à discuter; c'est en revanche la Gu qui est aiscutể a partir de cet a prior non empirique.

Cette conclusion est par consequent aifferente de celle qui a eté aegagée pax Botha, bien que nous arrivions tous deux a considerer la MS de la Formulation VI comme n'etant pas empirique. En effet, Botha, apres avoir examine une sexie d"exemples empruntês a Chomsky et Halle- et qui rentxent tous dans la FomulationVI

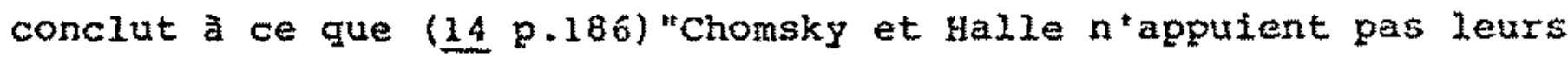
affimations sux le caractere naturel ou attendu des categories phonologiques, des regles et des systemes phonologiques, avec des faits dont la fiabilite et la pertinence puissent etre controlés. Par conséquent, il n'est pas possible de pretendre que ces affimations a emontrant la nature empirique de leur conception de valeur" (aeterminee, xappelons-1e, pax la MS).Or, cette constatation ne suffirait, dans notre cadre conceptuel, qu'a considexer la Is comme Eventueliement empitique.si nous la consiatrons comme fornelle, cest parce que, comme il vient d'etre explique, elle est posêe a priori et c'est a la GU de s'y adaptex.

La divergence avec l'interprétation de la MS pax Botha vient de ceci: la MS effectivement employée par chomsky et malle dans les exemples cites par bothe n'est pas la Hs dont il presente les trafts quelques pages auparavant, txaits qui tracuisent les declarations de chomsky et Halle sur la MS mais non 1 uzíisation qu*ils en font.En effet, Botha ( $14 \mathrm{p} .178$ ) indigue gue "la fonction de la mesure a'evaluation (=MS) est de selectionner la gxamaire evaluée comme plus haute, pour un langage, parmi les alternatives : gui ont Ete construites par application de la theorie linguistique gënerale aux donmes inguistiques".Cette Ms selectionme dono āes hypothèses en concurrence qui doivent rendre compte des menes donnés et cette exigence sur l'identite des donmes dont les $G$ 
doivent rendre compte est explicite dans les textes de chomsky et Halle (cf. 32 p.168 et, plus particulierement, p.251 et 331) cites par Botha; sa formulation de p.178 est par consequent en accord avec les textes originaux.Mais dans tous les exemples gu'il étudie (ibidem p.183-186) la hS a etê appliquee par les linguistes americains des $G \mathrm{~g}$ qui rendent compte des donnes diférentes. Par ailleurs, dans cette application de la MS- qui releve de la Formulation VI- ce n'est pas la MS qui est en 'jeu; c'est la Gu qui doit etre modifié afin que la MS -inchangêe- puisse définir conme plus simple les GP qu'elle determine et qui sont censëes etre plus"naturelies" ou posseder une generalisation(23).

La MS formelle de la Formulation VI introdult une singularité très importante parmi les MS formelles; les MS formelles des Formulations Ia IV definissent comme plus simpies des entités qui sont entièrement equivalentes ou équivalentes par rapport à un langage, que ces entites soient des GU ou des GP.Dans tous ces cas, Ia MS S'applique selon la tradition du "ceteris paribus", plus ou moins expliclte, dans le recours traditionnel a la simplicite, c'est a dixe, comme proposition non empirique venant a discriminer, dans une science empixique, entre plusieurs hypotheses concurrentes portant sur un meme domaine.or, dans la Fomulation VI, la wS joue un rôle infiniment plus important et qui, ef fait, fat sabirides distorsions au caractere empirique de toutes les hypothèses de la theorie.Ef effet, si l'on accepte la Formulation VI, il faut accepter que l'on ne puisse plus formuler les objectifs couramment annoncés en gramnaire generative;il faudra expliquer que la linguistique est, certes, le aiscipline qui se propose de rendre compte de la competence et de la faculte du langage mais gu'elle doit faire ceci par la formution a'une GU telle qu'elle déternine des GP definies dans certaines conditions comme plus simples que d'autres GP par une certaine MS postulee a priori.La MS devient ainsi une"règle de jeu" qui est. ci une part, impossible a justifier et qui, d'autre part, compromet le caractere empirique du reste des hypotheses.

La formulation.VI repond une lecture stricte des utilisations concrêtes de la MS dans un certain nombre de raisonnements clefs qui ont été fajts en grammaire generative pour 
justifler telle ou telle solution incorporce a la GU. On peut se demander si une lecture differente a'autres textes, qui ont des rapportshavec la formulation VI mais qui en different profondement, ne serait pas susceptible de faire mieux comprendre, sinon les utilisations effectives, du moins les intentions des linguistes américalns sur la simplicité.

Dans Aspects (20b p.64-66/20 p.43-44) on trouve un exemple factice comportant les éléments qui suivent:

GP-I (rend compte du Lang-1):Regle (15) possedant 4 symboles. GP-2 (rend compte du Lang-2):Règle (15-bis), non explicitée dàns le texte, possedant 20 symboles. GU-A:Rëgles syntagmatiques avec certaines conventions de notation (utilisation de parenthèses).

MS: non entièrement explicitée mals fondée sur le nombre de symboles utilises dans les GP.

Lang-1:item (16). Lang-2: item (17) .

Lang-1 et lang-2 sont diffetents mals possēdent le même nombre (vingt) de symboles.

On constate:a)GP-1 est spécifiée come plus simple que GP-2.

b) Le nombre de symboles de GP-2 est identique au nombre de symboles du lang-2.

On conclut: Nombre de symboles de GP-2 moins Nombre de symboles de GP-I= degré de généralisation de GP-1. Cette conclusion est explicitée dans le texte qui suit:

La notation parenthétique a dans ce cas la signification suivante: elle dit que la différence entre quatre et vingt symboles est une mesure du degré de genteralisation linguistique (significative-dang-l'original) qui est atteint dans une langue ayant pour le syntagme Auxiliaire les formes donnees dans la liste(16) (=Lang1), par opposition a une langue ayant par exemple, pour representer cette categorie, les formes de la liste (17) (=Lang-2)". 
C.. 7 De ce falt, I'adequation (1'edoption, dans l'origlnal) des conventions de rotations usuelles revient a poser que, sous-jacente à I'ensemble des formes de la liste(16) (=Lang-1), il $y$ a une généralisation linguistique (significative dans l'original) et que ce n'est pas vrai pour 1 'ensemble des formes de (17) (=tang-2\}.

Chomsky souligne que le fait que GP-1 possède une gentralisation par rapport a GP-2 est le resultat de l'adoption d'une certaine GJ-A caracterisêe par certaines conventions de notation; une Gu caracteriste autrement serait susceptible de deterainer la GP rendant compte du Lang-2 comme plus simple que la GP qui rend compte du Lang-1; on dirait dans ce cas que celle -la mais non celle-ci possede une generalisation:

"L'adoption des conventions de notation revient a formuler 1 hypothèse empirique que les regulartés du type illustré en (16) (=Lang-I) sont celles que l'on trouve dans les langues naturelies et sont du type attendu par les enfants apprenant une langue, et qu'au contraire, les rtgularites cycliques du type illustré en (17) (mLang-2), encore que prises abstraitement, elles soient parfaitement authentiques, ne sont pas caracteristiques des langues naturelles, ne sont pas du type que les enfants rechercheront intuitivenent dans le materiel linguistique, et pour ie sujet apprenant la langue sont bien plus difficiles a employer ou a reconstruire a partir de donnees disperses. Ce que $l^{*}$ on pose, dès lors, c'est qu'a partir d'exemples dispersës, tires de (16), Ie sujet apprenant la langue construixi ra la règle (15), engendrant l'ensemble entier avec ses interprêtations sémantiques, alors qu'a partir a'exemples desperses qui pourraient être regroupes par une rẻgle cyclique, il n'incorporera pas cette "generalisation" a sa grammaire.. .7 Il sexait aisé de suggërer une conven=tjon de notation différente qui abregerait la liste (17) en une regle plus courte que le liste (16), et,ce faisant, de formuler une hypothese de travail emplrique differente sux ce qui constitue une généralisation Inguistique". 
Si, suivant ces suggestions de chomsky, on modifie la GU, I"exemple prêcédent devient:

GP-3 (rend compte du Lang-1, Equivalente a GP-1): non- explicitée dans le texte.

GP-4 (rend compte đu Lang-2, equivalente à $G P-2$ ) : non explicitée dans le texte.

GU-B: non explicitée dans le texte; conventions ae notation differentes de celles ae GU-A et qui "favorisent" les xegularités cyclitgues.

HS: mon entièxement explicttee mais fondêe sur le nombre de symboles utilises dans les GF. Iaentique à celle de l'exemple preverlent.

on constate:GP-4 est specifiee comne plus simple que GP-3.

on conclut: GP-4 possède une généralisation.

Le texte précedement transorit de chomsky et celui qui suit insistent sur ceci: la conclusion selon laquelle GP-1 fqui rend compte du Lang-1) possede une generalisation et non pas GP-4 (qui rend compte du Lang-2) relève d'une hypothese empirique sur GU-A et sur GU-B:

"Pour rapprocher la théorie linguistiquue de l'adéquation explicative, nous pouvons essayer de raftiner la mesure d'évaluation des grammaires ou ae resserer les contraintes formelles pesant wur elles, de façon qu'il devienre plus difficile de decouvrix une hypothèse de valcux elcwbe qui solt compatible avec les données linguistiques primaires. Il n'est pas douteux que les théries actuelles de la grammaire ont besoin d'atre modifiass de ces deux manieres a la fois, la seconde etant en general la plus prometteuse" $6 . .7$ De cette façon, la theorie Iinguistigue pourra se rapprocher de $l^{\prime a d e q u a t i o n ~ e x p l i c a t i v e ~ e l ~}$ contribuer à l'etude des processus mentaux et de la capacite intel lectuelie de I home-pius précisement. à la determination des capacites qui rendent possible 1 apprentissage linguistique, eu egard aux limatation empiriquement constatés de tamps et de donnoses" (201) f.6. 120 P. 46-47). 
Dans quel sens peut-on dire que la conclusion selon laquelle GP-1 - et non pas GP-2- possède une géneralisation est une hypothese empirique? luel type de ronnëes peut tester cette hypothèse?Le texte cite ouvxe la porte a deux types d'observation

a) Ies régularités illustrées en (16) (=Lang-1) sont celles que l'on trouve dans les langues naturelies, contrairement aux régularitês cycliques illustrêes dans (17) (=Lang-2).

b) Les reqularites illustrées en (16) sont celles qui aeterminent un comportement particuliex au cours de l'acquisition

Il faut soul tgner que les observations de type (a) sont difficilement acceptables comme données: comment peliton déteininer l'existence ou non d'une régularite dans un langage? Pour ilistrer le problëme, soit l'exemple factice du Lang-2 présenté par chomsky.r1 obéit presque entièrement au schéma suivant:

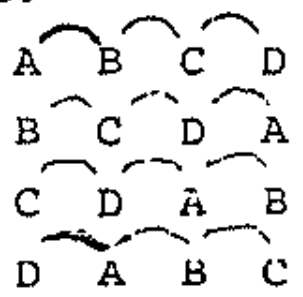

Or, on peut affirmer quion trouve ce type de langage cyclique dans les langues ou l'ordre des syntagmes n'est pas fixc, par. exemple 1'espagnol, où les énoncés qui suivent sont grammaticaux: la niñá comêun chocolatê en el campo come un chncolate en el camporia niña un chocolate en el campo la niña come en el camporla niña come un chocolate ("Ia Eille mange un chocolat à la campagne").

Mais il parait difficile que le fait d'attester l'exitence de ce langage cyclique valide empiriquement la Gu-B et la lis de l'exemple factice précêdent. Le texte de Chomsky doit donc etre compris comme exigeant, grur pouvoir conclure a l'existence a'une regu?arité dans; un langage, à la fois lobsarvation त'm tye caracterjsä de distribution des elements dans les suites du Iangage et aussi l'observation d'un comportement spécial 
de la part des usagers pax rapport a ces suites au cours de l'acquisition, en particulier, la fait qu'dis soient capables de matriser tout le langage a paxtir des"exemples disperses" * La formulation qui se degage de cet exemple factice ansi interpretée est donc la sulvante:

Formulation VI bis

Solt GU-A, Gui determine GP-1 et. GP-2.

GP-1 rend compte du Jang-1 et GP-2 du Lang-2.

Les etres humains dans le processus d'acquisition ont un comportement differencié par xapport au Lang-1 et au lang-2, la maj-trise du lang-1 étant susceptible a'être acquise a partir des exemples aisperses, ce qui n'est pas le cas du lang-2.

MS caracterise GP-l comme plus simple gue GP-2.

Par conseguent, GU-A et MS sont des hypotheses valides sur le plan explicatif et sont susceptibles de rencre compte de

I'accuisition du langage.

Si MS ne caracterisait pas GP-1 comme plus simple que GP-2, il Eaudrait:

(a) modifier la MS et/ou (b) modifier la GU et presenter une autre destinée comme la première à s'intêgrer avec une MS dans un modele d'acguisition ou (c) modifiex la gu et présenter une autre qui opererait sans MS ou (d) présenter un modèle tout a fait different de 1'acquisition du langage.

Dans la Formulation VIbis, MS et GU seraient des hypothẽses emplriques; elles auratent par ailleurs une interpretation psychologique dans le domane de l'acquisition du langage, cecl etant en rapport avec cela. En effet, la donnée clef gui rend la formulation VI bls empirigue, est l'observation du comm portement dans le processus a'acquistion: c'est cette adequation externe, accompagnee de la suppression du caractere priori accordêe a la MS, qui permet de parler de formulation empirique.or, le constat qu'on est amene à faire à propos de la Formulation VI bis est le suivant: nous ne connaissons aucun cas oi la formulation ait ete appliqué a une situation atteste Une premcondition indispensable poux qu'elle soit appliqué serait de definir les restrictions dans les rapports que 
doivent entretenir les donnêes differentes dont GP-1 et GP-2 rencent respectivenent compte; le même problème apparait aussi pour appliquer la Formulation VI et, autant qu'on sache, n'a jamis is ét posê.Mais par ailleurs, il existe une question plus gënerale et plus fondamentale: dans le cadre heuxisticométhodologique que la gramaire gênérative s'est donné pour envisagex Le probleme de I'acquisition du langage, la Formulation vi bis et le schema de recherche qui en dêcoule ne peuvent pas être appliqués. On presentera les fondaments de cette derniare affirmation au $\S 5$.

IV simplicite et empirisme.

Les Ms formeles éntuellement empiriques n'épuisent pas les MS de la gramaire génerative.Des essais d'utilisation de MS empixiques ont eu lieu et ils seront examines ici (Formulations VII et VxII). On domera d'abord, de lá première, une formulation gênerale; cellemci apparast appliquee selon des modalités difEérentes, qui seront prêsentées par la suite comme des types aifférentes de la formulation VII.La présentation de ces types sera précéeée d'un classement des données linguistiques qui permettra de les comprndre.pans la discussion de chaque formulation, on essayera de degager si elle est suffisament explicite pour etre appliquée et si, une fois appliquée et en tant qu'nypothèse empirique, elle a été validée ou infirmé. On présentera finalement la formulation VIII.

IV-I Formulation VII

Soit GU-A, qui détermine GP-1 et GP-2.

$\mathrm{GP}-2$ et $\mathrm{GP}-2$ ne sont pas entierement équivalentes mais elles sont Ecruivalentes par rapport à un Lang-1.

GP-I rend compte aussi du Lang-2, ce gue ne fait pas GP-2. HS caracterise GP-I comme plus simple que $G P-2$..

Par conseguent, GU-A et MS, determinant GP-1, gui est susceptible de projecter du Irang-1 au Lang-2, constituent un mécanisme de projection adequat. 
Si US ne caracterisat pas GQ i come plus simple gue GP-2, WS et/ou gu-a seraient infirmees et devarient etre modifiés.

cete formulation VII est fondee sur les textes qui sulvert: "Etant donne line telle thêrje, nous pouvons essuys: de construire des gramaires pour les languew existantes et nous pouvons. determiner si les grammatres les plus simples que nous pouvons trouver lc'est a dire les gramaires que Iatheorie generale nous contraint de choisir) satisfont ou non ax conditions externes d'adequation. Nous continuerons a reviser notre notion de simplicite et notre caracterisation de la forme des grammaires jusqu'i ce que les grammires choisias par la theorie satisfassent effectivement aux conditions externes" (29b $2.60 / 292.54)$.

"C...7 Mous essajerons de montrer que les gramaires les plus simples satisfont a certaines conditions externes d'adequator alors que les gramaires plus complexes, qui impliquent des decisions aifierentes relatives a 1 attubution de phrasis au noym, eto., ne les remplissent pas" (291 p.61/29 p.55).

"D'abord, nous essayons d'enricinir la structure de la theorie linguistique de maniare a restreinare la classe des gramaires qui sont compatibles avec les donnees presentês- en d'uutres termes, nous essayons de faire I'affirmation universelde plus forte et legitime sur la structure du langage. Deuxiemenent, nous essayons de construire une procedure d'tuluation suscoptible de stectionner une parni la pluralite de grammaises permses par la thorie linguisticue propose et compatibles avec les donnes prescntés"(31 p.107). "pour construire un modele ratsonnable de l'acquisition, ji est necessaire de reduire la classe des graminatros a la fols réalisables et compatibles avec les donnes linguistiques primains, jusqu'au point ou nous pourrions fatre usage, pour choisit entre elles d'une mesure formell a evaluation (20b p.56/20 p.35). 


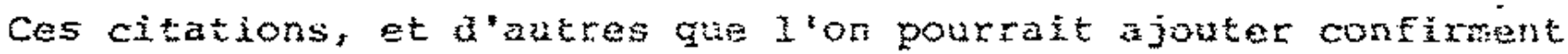
les points essentieis de la forrulation VII les ge conparés doivent rencre compte d'un mâme ensemble de donates et etre

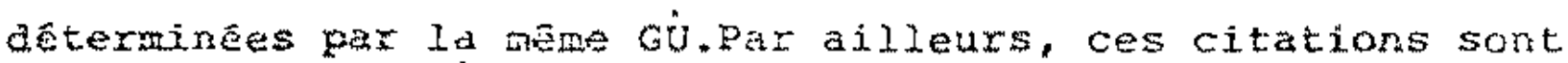
erglobés dans la problematique de l'adequation explicative de la theorie linguistigue, probicnatique qui conncide, aans loptique chorikyenne, arec ceIle de l'acguistion du largage.c'est d'une paxt ce caju-là et d'autze part, les utilisations concrêtes à cete HS, gui autorisent d introduine les autres bxaits propres a la Forrulation VII et notament, la capacite projoctive de la HS operant sur des GP determines par une Gu donné (25) . En effet ia us et la Gu constituent une machine de projection", cellewi. pouvart se definit comme une machine gut ręoit comme entro des données d et qui produit come sortie une GP susceptible de renoxe compte de det, en pus, d'autres donnés a"; les donnés d et $\mathrm{d}^{\mathrm{r}}$ sont incluses ans une classe $\mathrm{D}$ et 1 intersection de a et $\underline{a}^{*}$ est nuile.Les donnes d et $\mathrm{a}^{\prime}$ seront respectiverit. nomees donneses originales et donnes projectives.ix is et Ia ou sont une machine de projaction car elies pexmetient d'obteniz une GP susceptible de rendre compte des données qui leur sont prasentes (connées originales, le Lang-1) et, en pius, d'autres donnes (connes projectives, le Iang-2).

is et GH etablissent donc, compe toute machine de projection, ur rapport entre les donnés originales et les donnés projectives.une manjexe de caractécisor les différents doublets

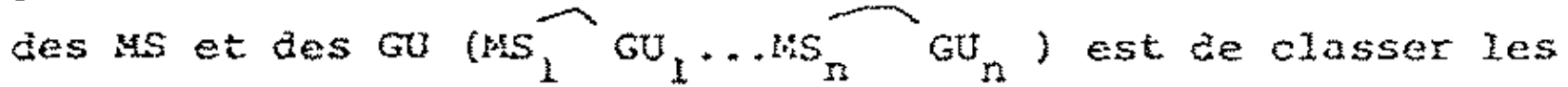
types des donates originales et le type des donnes projectives entre lesquelies chaque Ms Go etablit un rappoxt.ceci exige un classement des ionneses dont une $G P$ doit renare compte.

Nous astinguons a ax types de donnees lingustiques: Ies données observables et les donnees profondes Donnëes observables sont celies qui se revelent dans les signifiants des enonces et peuvent pax conséquent être captées a partir des indices perceptibles Tout ce gui a trait aux "sons", au sens le plus large, relète aes donnêes observablest les unites phoniques eliesmênes, màs aussi leur linéarite. l'agencernent des unités 
phoniques en signifiants, la Iinearite des signifiants, ctc. Les données observabies n'exeluent pas les considerations sux la signifié, pourvu que colles-ci soient rattachabjes à des differences perceptibles dans le sigrifiant. Les jugements de grammaticalitê, d'être identique ou aifférent à l'audition, ce deviation morphologique ou syntaxique en surface, sont autant des données observables.Les annēes profondes sont celles qui ne sont pas susceptibles d'être captếs par un processus perceptif ou par l'observation des signifiants des enonces. Les jugem ments d'ambiguté syntaxique, didentité de paraphrase et/ou des rapports syntaxiques non manifestes en surface, sont des donnés profondes.Les données profondes, en lignes günérales, sont celles qui ne respectent pas la consigne structuraliste selon laquelle a toute difference de sens devrait corresponare une difference de son.Ceci est typiquement le cas des ambigurtés syntaxiques, car aucune distinction dans le signifiant ne correspond à la distinction de signifie propre à l'ambiguIté. Les donnés profondes ont une relation avec les donnes observables corresponiantes; neanmoins et par la détintion de la notion même de donntes profondes, l'observation des connees observables qui véhiculent les donnêes profondes, ne permet pas de determiner i'existence de celles-ci.par exemple, dans "Il m'a expédie le message de la pharmacie", rien, dans le signifiant de la phamacieou dans le reste de i'enonce, ne permet de concluxe a l'ambiguaté de l'énoncé et, en particulier, à l'ambiguite des rapports syntaxigues de de la pharmacle.Mais cette donnée profonde gu'est I'ambigurté syntaxique, $a$, cependant, une relation avec le signifiant qui la vêhicule, cest à dire avec il m'a expédie le message de la pharmacie. on nommera "donnee observable correspondante" la donnee observable qui est en relation avec une: connee profonde. on établit la convention que l'expression donnees profondes désigne et les donnees profondes et les donnés observables correspondantes.

La categorisation en donnés observables et en domees profondes se superpose avec la catégoxisation on données originales et donneres projectives. 
on a,par consequent, las quatre types de comeates qui suivent:

A: originales et observabies

B: originales et profondes

C: projectives et observables

$D:$ projectives et profondes

Ia projection doit donc s'effectuer entre donnés de la catégorie A ou B et donnés de la catégorie C ou D.Or, la categorie $D$ peut se subdiviser en deux sous-categories. En effet,on pout distinguer les deux situations suivantes:

a) Situation $D-1$ ou de coincidence des donees observa-

bIes.

Les donnét observables correspondantes (c'est a dire les données observables gui sont en relation avec les connees profondes) sont les memes donnês observables que celles de donnés originales a partir desquelies la projection s'est effectuée.

b) Situation $\mathrm{D}-2$ ou de non-coincicience des données

observabies.

Les données observables correspondantes ne sont pas les mêmes ännées ubservables que celles des données originales a partir desquelles la projection s'est effectuée.

on a par consequent:

données

originales

Situation D-I W projectés en

Situation $D-2 \quad W \quad$ projectées en $Y$
Données

observabies correspondantes en

N

$\Psi$
Données profondes projectives 2 $z$

Il - est possible, à partir de ce classement des donnés, de caractêriser les différents doublets de. MS et de GU en tant que mécanismes projecteurs. Chague MS GU sera caractérisé par le type de donnëes originales et le type de données projectives qu'il met en rapport.or, nous avons pu repérer, dans les écrits de Ia gramaire générative, au moins trois types de projection differents ou mention explicite est faite du role de la MS: 
rype $I$ : De donneses a (originales et observables) a donnés C (projectives et observables).

Type II: De donnés A (oziginales et observables) à donnés D-1 (projectives profonaes et coincidence des donnes observables) rype III: De donnees A (originales et observables) a donnees D-2 ( projectives profondes et non coincidence des donnés observables).

cinaque type de projection definit une modalité differente de la Forrulation vri.ces trois modalites seront successivement examinées par la suite.

\section{IV-1-1 Formulation VII-Type I}

Le xypel de la formulation VIr apparat en relation avec les variations morphologiques des matrices phoniques d'un même fomant.Les connês A (obaervables) sont constitués par les variations morphophonologiques régulieres constatés dans certains contextes et pour cetains items lexicaux; les donees $c$ (projectives) sont constituees par a'autres variations morphophonologigues qui apparaissent dans d'autres contextes, ces nouvelies variations et/ou ces nouveaux contextes n'ayant pas ete constates parmi les domnées A.Ie texte de Chomsy (21 p.65) gui suit, illustre cette possibilite:

"La gramaire parvienarait au niveau encore pus haut d"adequation explicative/...7 si la thérie inguistique gij 1 in est associêe etait susceptible de pourvojr une structure pour les règles phonologiques et une mesure d'êvaluation satisfaisant la condition suivante: l'ensenbie de regles evalue comne plus haut et de la forme appropriê, ayant etê selectionme pour engendrer un ensemble d'items où les variantes de "telegraph" sont exclues, serait l'ensemble de règles qui en fait predit cette variation contextuelle de "telegrapin"."

Nous ne connaissons aucune formulation explicite de NS et de GU ồ cette possibilite ait ate concretisételle est plus un programe de formuation empirique qu'une formulation expldaite; 
impossible dono de controler-si, dans un certain nombre de cas, une hypothese quelconque relevant de cette cormulation se verifie ou non.

Le Type I de la Formulation VII apparart aussi ailleurs, mais explicite dans une formulation suffisament claire pour etre teste. Il est applique au probleme de sequences phoniques non attestếs mais possibles. En anglais, par exemple, brick, blik et bnik sont, respectivement: (a) une sequence phonique attestée dans un corpus de 1 anglais; (b) une sequence non attestee mais possible;blik est le signifiant d'un mot qui n'a pas de signifié mais qu'un anglophone admettrait come sequence possible, susceptible d'appartenix a l'anglais et de recevoir un sens; $(c)$ une së́cuence non attestée mais impossible, bnik n'etant pas accepté par un anglophone comme le signiflant d'un mot possible dans sa langue.par defintion donc, aussi bien les séquences non attestees possibles, ou absences accientelles, que les séquences non attestées impossibles ou absences systematiques, ne peuvent pas appartenix aux donnes obscrvables: ceci pose le probleme de projeter la distinction entre absences accidentelles et absences systematiques a partir des sequences attestees.

Le doublet MS GU, suftisament explicite et qui a ête proposé en grammaire generative pour rasoudre le probleme ainsi pose, presente les caracteristiques suivantes:

GU: (a) le signifiant des formants du lexique est defini par une matrice de traits distinctifs binaires.

(b) Les règles de reforance lexicale incorporent aux traits des matrices lexicales des traits redondants; ces regles ont, d'une part, certaines capacités formelies, et d'autre part, operent sur des traits distinctifs binalres.

NS :GP-I est plus simple que GP-2 si le nombre des tratts distinctifs binaires utilisés aussi bien dans la spectication du lexique que dans les règles de redondance est moins eleve dans $G P-1$ que dans $G P-2$,

Le mecanime de projection, dans cette hypothese de dou-

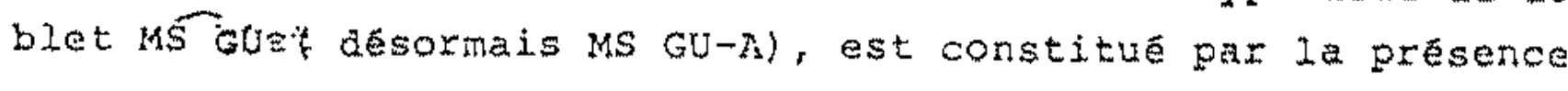
ou abscree d'une ragle de redondance.par exemple, soit les règles 
(empruntees chomsky et falle, 31 p.101)

(1) Segment consonantique-_-_Liquide ano lo contexte: $\$$ occlusif-voyelle

(2) Segment consonantique- $\longrightarrow / \mathrm{r} /$ danis le contexte: $\mid \mathrm{b}-\mathrm{ik} /$

La premiere règle exprime le fatt qu'entre consonne occlusive initiale et voyelle il $n^{\prime} y$ a en anglais que $/ r /$ ou $/ 1 /$; elle permet d'épargner, dans le lexique, la specification do "Liquide" du deuxième Elément phondque, pour tous les formants tels que craft, blend, prize, etc.ta deuxième règle exprime le fait qu'entre $b$ et $\underline{i k}$ on ne trouve que $/ x /$ dans le lexique anglais; par là même, elle exclut /blik/ parmi les séquences non attestêes mais possibles.Les deux rẽgles sont adéquates par rapport aux données originales et observables, mais c'est la seule regle (1) qui tetabit la projection de manize adequate n'excluant pas le cas du possible blik, ce qui, en revanche, fait la règle (2). C'est donc la presence ou l'absence d'une rêgle de redondance qui conditionne la projection des stquences non attestêes possibles.

Les règles de redondance operrent conjointement avec un lexique, et un même langage peut etre speciflé modifiant soit le lexique soit les rẹgles de redondance, soit les deux, les GP qu'on obtiendrait modifiant ces elements pouvant être equivalentes par rapport à un langage determine.par exemple, dans le cas de 1'exemple suivant, on aurait:

$$
\text { GP-1: }
$$

(a) Lexique sans indication de "liquide" dans le contexte: consonnemvoyelle.

(b) Règle de redondance(1).

$$
G P-2 \text { : }
$$

(a) Lexique avec indication de liquide pour tous les formants dans Ie contexte: consome-voyelie.

(b) Aucune règle de redondance Equivalente à jis (i). GP-1 et GP-2 sont equivalentes par rapport à un langage constitue de séquences douées de signifié. 


$$
\text { GP-3: }
$$

(a) Lexique avec indication des taytes specifiques de /r/ dans la matrice de brizk-

(b) aucune regle de redondance equivalente la regle (2).

$$
\text { GP-4: }
$$

(a) Lexique sans indication des tratts spécifiques de/r/ dans la matrice de brick.

(b) kêgle de redondance (2).

GP-3 et GP-4 sont equivalentes par rapport 3 un langage constitue de secuences doutes de signifié.

Les situations 1 ilustrees par les paires GP-1 et GP-2, GP-3 et GPw, contituent un cas tipioue ou la ks aevrait operer.si on applique celle gui a ete proposee dans MS GU-A, on observe gue GP-1 et GP-3 sont correctement caracterisees comme plus simples que, respectiveruent, GP-2 et GP-4. En effet, dans le cas de GP-1 et de GP-2, le nombre des tratts gue l'on doit utiliser pour expliciter la regle (L) est moindre que le nombre des traits apargnes ans le lexigue; la ks oblige donc a incorporer la réle, ce qui, comme il a te remarque, est adgquat. En revanche, dans le cas de GP-3 et de GP-4 le nombre des trajts que 1 'on doit utiliser poux expiciter la regle (2) est plus eleve que le seul trajt epargne dans la matrice phonique du formatif brick:par consejuent la règle de redondance (2) ne doit pas etre incorporée.

La ils du doublet MSGU $\mathrm{A}$ est donc empirique: la donné qui servira à la verifier c'est la possibilite de distinguer les absences accidentelies des absences systematiques, pari les sequenzes qui n'ont pas eté observês.

Nous n'avons pas connaissance d'un processus actif de recinerche destine à proposer des gramaires particulières que la Nis du doublet MS GU-A devrait caxacteriser comte pius simple. Exception fatte de quelgues exemples simplifies a lextrene, nous n'avons pas connaissance dautres cas ou cette MS ait ete appliquëe (25). Mass le fait qu*elle soit suffisamment explicite et 
expirique permet de la tester.

II est relativement facile de trouver des contre exemples qui infiment la capacite projective de MS GUm. En voici trois, dont le premier est emprunte au français et les deux autres à I'espagrol.

II se trouve que le lexique du français est faiblement reprédenté dans le contexte chu- ou chou-; en revanche, les initiales su- et sou- abondent. Cette observation permet de formuler la règle de redondance suivante, dont nous donnons deux formulationg, l'une plus technique que l'autre:

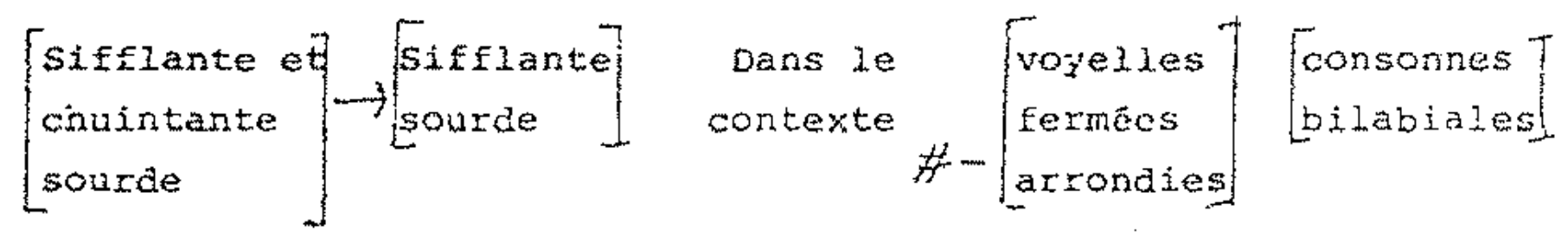

$\left[\begin{array}{l}- \text { voc } . \\ + \text { cons. } \\ + \text { cont.. } \\ + \text { tendu } \\ - \text { grav. }\end{array}\right]$

$$
\left[\begin{array}{l}
+ \text { voo. } \\
- \text { cons } \\
+ \text { diffus bert. }
\end{array}\right]\left[\begin{array}{l}
- \text { voc. } \\
+ \text { cons. } \\
- \text { comp. } \\
+ \text { grav. } \\
-\operatorname{cont} .
\end{array}\right]
$$

cette rẽgle comporte 15 traits; dans le dictionnaire consulte, le lexique du frangais ne comporte pas moins de 190 items

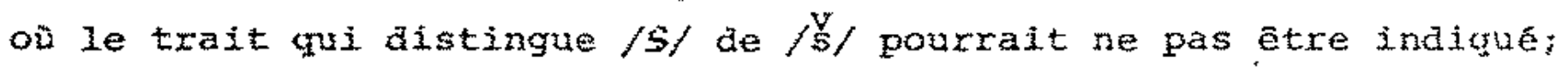
elle devrait par consequent, selon MS GU-A, etre incorporê. Or, cette rëgle classerait, par ex. les sëquences choupette, choumer. chumex, etc. parmi les non possibles, ce qui est inadéquat (26).

Le lexique espagnol présente peu de $n$ initiales suivis de a ou de e et a'une consonne nasale.en revanche, on compte envixon une centaine a'items avec $\underline{t}$ ou $\underline{d}$ dans cette position.on peut donc formuler la rẽgle de redondance suivante:

$\left[\begin{array}{l}\text { consmrines } \\ \text { dentales }\end{array}\right] \rightarrow$ [orales $\left.] \quad \begin{array}{l}\text { Dans le } \\ \text { contexte }\end{array} \#-\left[\begin{array}{l}\text { a } \\ \text { e }\end{array}\right]\right] \quad\left[\begin{array}{l}\text { consonne } \\ \text { nasale }\end{array}\right]$ 


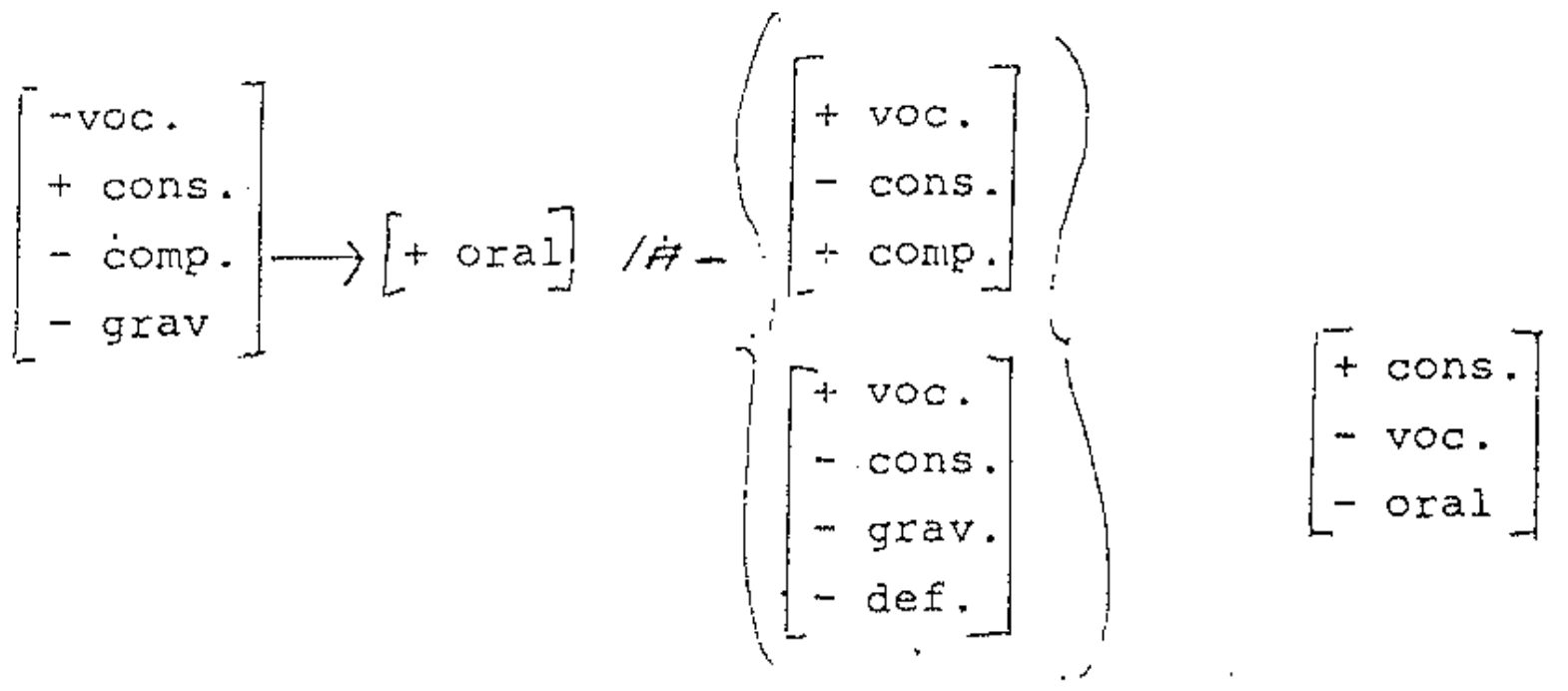

cette règle, qui comporte 15 traits, devrait être incorporécependant dans les données prijectives, elie classe nanzarina, nentro, nentáculo, nemplado, etc., comme des absences systênatuiues, ce cqui est inadéguat.

Le lexique espagnol présente peu de sêquences initiales is ou inis;en revanche la séquence es initiale et précedant une consonne vêlaire est abondamment représentée (190 entrées).

La rogle de redondance suivante, qui comporte 15 traits devrait par conséquent etre incorporêe:

$\left[\begin{array}{l}\text { voyelies } \\ \text { antérieures }\end{array}\right]\left[\begin{array}{l}\text { voyelle } \\ \text { anterieure } \\ \text { mi-ouverte }\end{array}\right]\left[\begin{array}{l}\text { dans le } H-[5] \\ \text { contexte }\end{array}\right]\left[\begin{array}{l}\text { consonne } \\ \text { vélaire }\end{array}\right]$

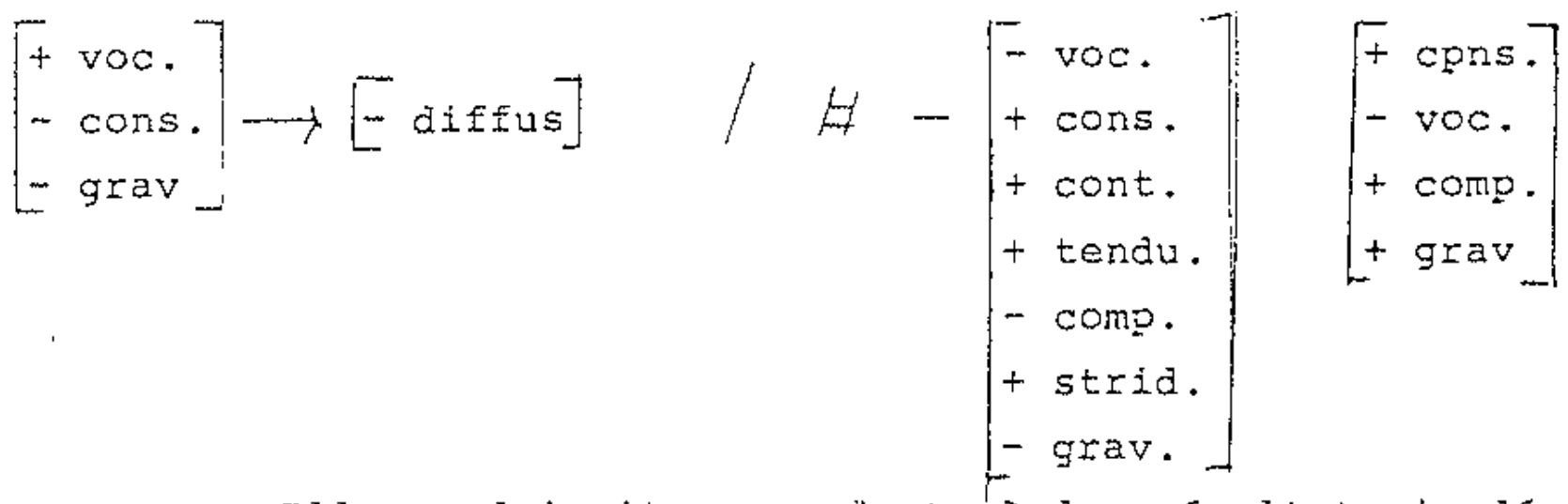

Ele conduirait, cependant, a des resultata inadequats, car des séquences telles que iscala, iscabeche serraient ciassetes dans les données projectives comme des absehces systematiques. 
MS GU-A est 12 seule nypothese explicite que nous connaissons dans le cadre de la Eormulation VII-Trpe I Dans la mesure ou les seuls cas connus henant des résultats corrects ne sont wue des exemples lawgement articiciels, ot come dans los trois cas approchant de la complexté de deux langues naturelies elie s'est revélée fausse, on peut conclure que MS Gu-A est une iypothése gui est, cextes, empirique mais, pour i'instant, complètement infixmée $(27)$.

IV- I-2 Formulation VII-Type II. Le Type II de la Formulation VIIde connées A, originales et observables, à des donnếes $D^{-1}$,

donnees projectives, profondes qui pressentent une comodidence ma des donnes observables- apparatt utilise cans certains traitements de 1 'ambiguztë pt, plus generalement, dans des discussions sur le rapport entre structure de surface et structure profonar. Dans (293 p.97-98/29 p.88-89), Chomsky remarque gue l'enonce (111) The shooting of the huntexs peut etre compris comme possedant le schama syntaxigue de (112 i) The growling of lions ou celui de (112 ii) The raising of flowers.Et il ajoute:

" En termes transformationnels, cependant, I'explication est claire et automatique. Une analyse precise de l'arglats montre gue nous pouvons simplifier la gramaire en roje tant des syntagmes du type (111) et (112) hors du noyau et en les réintroduisant par une transtormation. Si lon procède de cette manière, l'énoncé (111) The shooting of the hunters aura deux origines transformationnelles distinctes; il ara une representation ambigü au niveau transformationnel".

L'observation transcrite peut se reformuler ans les termes suivants: si l'on utilise une u avec une composante de transformations, on peut verifier que la gramaixe plus simple qui rend compte de la grammaticalite ae (111), (112-i) et (112-ii), ces suites étant consideres parmi les donnês origineles et 
observables, élimine ces enpneés de l'ensemble des ënoncts noyaux et les specirie au moyen des transtornations.or, ce faisant, elie assigne une double strueture au signifiant(1I1), rendant ainsi compte de son ambiguIté.C'est-àmuire que la GP caracterisée comme plus simpic rend comple non seulement des connecs originales et observabies portant sur la grarndicalite des suites (1I). (121) et (1l2 ii) mais,aussi, de i'ambiguate de (111), c'est a dire des données protondes dont la partie observable conncide avec les donnés originales et observabies. La GP determinee par cette GU et caracterisec comme plus simple par la kS, est suscepthole de projecter des donnees $A$, portant sur la gramaticaliter des suites (111), ( 112 i) et ( 112 i. $)$ i des donnes $D-1$, portant sur l'ambigurtê de (111); elle ajoute donc de la "profondeur" aux donnees observabies.

Dans $21(0.66-67)$ réapparast un rajsonnement analogue au cours da la discussion de (3) Eohn is easy to piease et de (4) John is eagex to please. Chomsky observe que le niveau a adécuation explicative serait attaint si I theorie était susceptible de Stictionner une gramaire adequate sur le plan descriptif stant cionne les enonces (3), (A) et d autres suites qui ont un rapport avec elx. L'adquation descriptive conchant largement avec ia notion de dométs profondes, on peut legitimement supposer que L'on demande à la GP de rendre compte du niveau profond des enoncés (3) et (4) lorsqu'on ne dispose comme donntes origineles que de la partie observabies de ces mêmes enoncés:

"I y a de bonnes raisons de penser cue dans les cas de (3) et de (4), la theorie de la gramaire transformationnella peut s'approcher du niveau de l'adequation explicative et présenter une explication paxtielie de l'intuition Innguistique du iocuteur.c'est a aire que la grammaire qui assigne les descriptions structurales correctes possède des generalisations qui ne sont pas exprimées par les grammaires qui ne pourvoient pas les descriptions structurales correctes et est par conaéquent êaluee conne plus halite (c'est a dire,plus simple) cans un sens gue I'on peut 
apparement presiser sans beaucoup de difficulté" (2lt 67).

Mis à part les textes qui procedent, largenent programm maticues, on ne comait aucune formuation explicite de MS, ans le cadre de la Formulation VII-Wype II, pernettant d'operer conjodntement avec I'une des GU proposés en grammare générative. Il a donc eté impossible de jegager un doublet MS Gu suffisamment clair-conne il etait le cas dans la pormulation precedente- pour etre testé.

\section{IV-1-3 Formulation VII-IYpe III.}

Le rype III de la formulation VII- de données $A$, originales et observabies, a des comees $D-2$, projectives profondes sars conncidence des domnés observables-m est suggerë dans le texte qui suit:

"Supposons gue nous avons une grammäre transformatinnelle

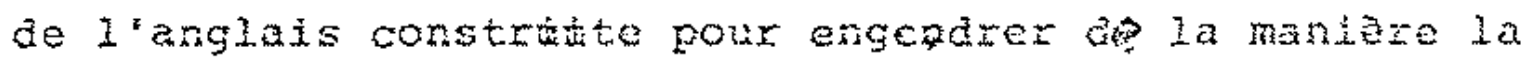
plus economigue I'evantai complet de constructons ajjectives a 1 'exclusion de comparatives on peut montrer (renvoi a Cis.smith, "A class of Complex Modifiers in English", dans targuage $37(1961)$, p.342-365) gu'une large variete do constructions comportant des comparatifs seront engonareas automatiquement par la grammaixe aveo la disposition correcte des ambiguitos, cas purticuliers et "exceptions" apparentes, si l'on ajoute a la grammire au point approprié dans la sequence des règles ordomés, 12 transformation genexalisee qui forme la construction comparative la plus simple(...) c'est par conséguent ur cas intéressant ou l'on peut dire que la theorie gênérale de la gramaire transformationnelle offre une explication pourun amoncellenent de donnees de surface assez desordonnées" $(21,0.68)$.

Le texte qui prểe s'interpréte de la maniere suivante: a partir des donnees originales et observables (des suites qui correspondent aux constructions adjectives et a la comparative dans sa forme plus simple) la GU et Ia MS proporées dans le cadre 
de la gramaire génërative sont susceptibles de proposer une te telle qu'elie doit rendre compte des connées précédentes et, en plus a'autres données profondes monme les aublgurtesm et ceci aussi bien pour les donnes $A$ (construtions adjectives et la forme la plus simple de la comparative) que pour les autres domnés projectives (les autres onmaratives que les plus simples). L'interpretation que Fodor, Bever et Garrett '(39b) donnent du modèle a'acquisition du langage présenté par Chonsky dans Aspects (cf. 20 p. $30-31 / 200$ p. 50-51), modele qu'ils jugent inadequat, rentre dans les ternes de ce type III de la Formalation VII.

Homis la suggestion du texte prêcedent, on ne connait aucune formulation explicite de GJ et de MS susceptible d'être test.ée.

\section{4-2 Formulation VIII}

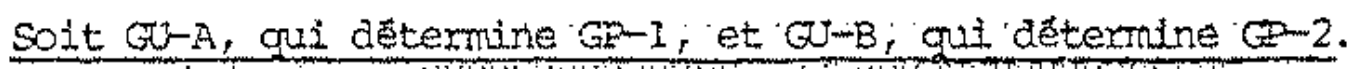
GPal et GP-2 ne sont pas ent lerement Equivalentes ma is elles sont EquivaLentes par rapport a lu Lang-1.

GP-1 rend compte du Lang -2 ; ce gui n'est pas le cas de (qu-2. WS caractérise GP-1 corme plus sincle que GP-2. Par consécuent, GU-A doit etre prêFerée à GJ-B; et GJ-A et MS sont validées GP-l étant susceptible de projecter du Irangir au Iand-2.

Si MS me caractérisast pas GPin conne plus simple oue GR-2; MS et/ou Gr-A seraient infirmées et devraient être modifies.

Cette Formulation VIII est, come la precedente, enpirique, mais à la differrence de la Fomulation. VII, les GP contareses par MS sont determines par des Gu differentes. Elle a ett appliquée par Halle dans le donaine phonique, plus précisêment dans les règles accentuelles de l'anglais (49 et 46) ${ }^{28}$. La presentation qu'on put en degager est la suivante :

Gi-n : les rêgles sont organisees selon un ordre disjoint.

GJWB : règles non ordonnées.

G-1 : item (6) dans une version non fomalisée et item (9) dans une version formalisêe.

GP-2 : Ltem (3) dans une version non formalisee et item (4) dans une version formalisée. 
Iargml : liste des mots présentes dans l'iten (2), qui componte 23 nots. Lang-2 : (a) mots du type yenom, avec voyelle accentuee non tenaie, gui recolvent l'accent princlpal dans l'avant-demière position (itent (12), Gui comporte 6 mots).

(b) monosyllabes avec voyelie non tendue, gui deviennent accentuées.

(c) nots di type suez, qui reçoivent l'accent principal dans la dernière syllabe avec voyelie non-tendue (item (13), gui comporte 6 mots).

MS : exprime dans l'item (11) par le terme "prolixite" ; elle porte sur le nanbre de symboles.

La projection de type (c) dans le Iang-2 ne peut être atteinte grie si l'on ajoute à GP-1 (constituëe par I'ttem (6) ou (9), une "rêgle d'exception", non formulee dans le texte, mais qui, par les commentaires gu'on y trouve, profite de I'ordre disjoint de GP-1 : si un mot est maxque comme exception à ure des règles quil sont abrëgês dans I'item (9), on lui applique automatiquement la règle suivante. 0 a par sinsiquent: GP-1 bis : GPul (mitem (6) ou (9)) plus règle a'exception.

Aussi bien Gew (et GP-1 bis) que Gp-2, rendent conpte du Lang-1, c'est-à-dire qu'elles placent $I^{\prime}$ accent principal dans les mots anglais du Lang-1 en tenant compte des caractéristiones phoniques des segrients qui camposent leurs signifiants et en pirticulier, du carecm tère tendu ou non des voyelles dans les sucoessions des voyelles et des consonnes 29 . mrois positions ont été prérues à partix de la dernière syllabe; elles apparaissent dans les exermples qui suivent, erpnuntés au Lang-1 :

Posit. 3

Posit. 2

Fosit. 1

America

Arizona Wisconsin

Tennesse

aluminum

arboretum

carbornandum

suprene

Pour pourvoir considerrex que $Q J-A$ avec MS sont verifiés, 11 faudrait ne pas pouvoir accurnilew les contre-exerples. Or, la variete des contremexemples est si grande, ils sont si faciles à trouver, parce que 


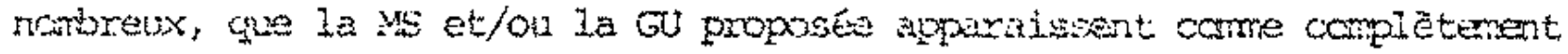
infimies. Et il purait irpossible de considerer ces contre-exerples come des "exceptions", quelle que soit la notion d'axception qu'on se donne. on peut en dégàger trois types :

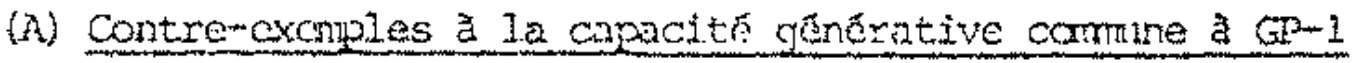

Let à GP-l bis) et $\mathrm{a}$ Ge-2. Ce sont les cas ou l'accent principal apparait place en dehors des situations attestëes dans le Lang-1. Toutes les possibilites continatoires de contre-exerples possibles sont attestees pour les trois dernieres positions; en plus, on trouve des contre-exerples dans bes ribts qui piacont l'accent principal en ahors des trois demieres positions prérues:

(A-1) Bosit. 4 attestee, alors quo posit. 3 ou 2 ou 1 prévies : dedicatory degeneracy

(A-2) Posit. 3 attestee, alors que posit. 2 prevrue : concretely Elypancy

(A-3) Posit. 3 attestze, alors que posit. 1 prevrue (une règle d'antéposition, que I'on peut ajouter aussi bien a (PD-1 (et à GP-Ibis) qu'a $G$-2, rend compte de ces cas) :

$$
\text { concretize concubine }
$$

(A-4) Posit. 2 attestee, alors que posit. 3 prévue : flotilia colition

(A-5) Posit. 2 attestêe, alors que posit. 1 prêvue : Eellow Frmale

(A-6) Posit. 1 attested, alors gue posit. 3 prêrue : convalesce clarinet

(A-7) Dosit. I attestée, alors que posit. 2 prévie : drawee convise

(B) Contre-exemples à la capacité gênérative particulière à Gpu I (sans règle d'exception). Ce sont les cas ou l'accent principal apparait placé en clehors des sttuations attestees dans les formes (a) du lang-2 (mots du type venan):

$$
\text { canaI : cadet }
$$

(C) Contre-exerples a la capacité generative particulière à GPulbis. Ce sont les cas où, a la diffexance des situations attestées dans les formes (c) 
du Iang-2, Ia règle d'exception ne produit pas des résultats adéguats :

\begin{tabular}{|c|c|}
\hline $\begin{array}{l}\text { Porme attestee : } \\
\text { feline } \\
\text { glucose }\end{array}$ & $\begin{array}{c}\text { Devrait etre sejon } \\
\text { GPul et G }-2: \\
\text { feline } \\
\text { glucose }\end{array}$ \\
\hline deftciency & deficiency \\
\hline Copulative & copulative \\
\hline crematory & crematory \\
\hline convalesce & convalesce \\
\hline
\end{tabular}

Devrait were selo.
cp-l bis :
feline
glupose
ceficiengt
copulative
creratory
corvalesce


L'interpretabilite psychologione des focmulations empiriques.

I' abscence d'un processus de formilations empiriques explicites sur la simpliclté et le fait que les seules qu'il a ete possible de tester soient infirmés, est a rapprocher d'une autre constatation : ceux qui, dans le cadre de la psycholinguistique gentrative ont aborcé le problètre de I'achulisition du Iangage, n'ont pas indopore une MS explicite et empirique aux propositions qu'ils ont faites pour expliquer le processus a'acgulsition. Cette onission est d'autant plus remarquable que dans la vision chansikyenne au probleme -dont ils se réclement- la simplicite est the piêce essentielle $\mathrm{e}^{30}$. Ces deux constatations, bien que difiérentes, sont en rapport entre elles.

On a pu constater au $\S 4$ que les WS empiriques, aussi bien celles qui ont eté explicitées que celles qui sont suggerées, sont destintes a etre integrées dans un mécanisme projectif. Or, $11 y$ en a plusieurs trpes de projection possibles; ci-dessus on'n'a presente que les modalites inaginées en fonction d'une typologie posere au depart : rien $n^{2}$ enteche d'inaginer beaucoup d'autres molalités difficrentes. Celles-ci peuvent être toutes mpiriques, dans oe sens qu'il sera toujours possible de vertifier si la GP caractérisée come plus sirmple est susceptible ou non de rendre orwte $a^{\prime}$ un certain type de données projectives a partir d'un certain type de donnees originales. Il se pose dono un probleme methodologique capital : laquelle de ces molalités de projection choisir et pourqui ${ }^{31}$ ?

Nous crovons que seules delux rëponses possibles existent : ou bien il faut choisir arbitrairement ou bien il faut adnettre que la solution choisie doit être interpretable psychologiquenent et devenir, par consequent, the hyothese emixique dans $I^{\prime}$ acquisition cu langage. Ie choix arbitraire implique que la linguistique ne sera qu'me discipline partieliement empirique : des "rëgles de jeu" non motivées, devraient être introduites dans son cadre méthodologique pour restreindre la multiplicite des possibilites descriptives. On cherchera, p. ex., le doublet $\mathrm{MS}^{\wedge} \mathrm{GJ}$ susceptible de projeter des données originales et observables. à des données projectives et profondes sars colncidence des données observables, ou bien des donnes originales et observables qui ne supposent pas l'utilisation do symbles recursifs à des données projectives et observables qui supposent un nombre $X$ de degrés d'autoenchassement, etc. I' inagination est fertile et peut 
suggerer beaucoup de posstbilites : On risque, certes, de s'approcher encore plus au cas limite vers lequel on glisse doucement deja, ou chaque linguiste proposera le type de projection cui lui convient. Il y aurait alors autant de Iinguistiques que de Iinguistes ; toutes seraient enqiriques mais avec des règles de jeu differrentes.

L'tnterpretabilite psychologique constitue une solution possible au probleme du choix du type de projection ; elle implique de replacer le probleme des descriptions linguistiques dans le cadre des hypotheses sur les conduites Iinguistiques, dans ce cas particulier, les conduites linguistiques d'acquisition. Une machine de projection peut, en effet, ettre une hypothèse empirique interpretable psychologiquement dans le cadre de l'acquisition du largage, car elle peut résoudre le probleme de l'accuisition, à partic a'un langage fini, des structures finies permettant de rendre compte $a^{\prime}$ un lagage infini. Dars ce cas, le type de projection deviendratt à son tour une hypothèse empiricue : à tel stade du developpenent de 1'acquisition, on devrait chercher à présenter des hypothèses pemettant de projeter tel type de domées à tel autre type, car on aurait constaté que ces donnees sont effectivement et respectivenent les donnes d'entrée pour l'enfant. (ies donnés originales) et les donnés sur lesquelles il possëde une comaissance intuitive (les donrées projectives).

pourquol, malgre la prétention à l'interprétabilite psychoIogique des hypothèses sur la Gu, et, plus particulièrement, dans le cadre de ce travail, de la $\mathrm{NS}$, rlen a rotre conaissance $\mathrm{N}^{\prime \prime}$ a été fait dans un schena de recherche visant a expliciter, tester, vallder ou infirmer des hypothèses sur la MS, empiriques et interortables psychologiquement ${ }^{32}$ ? Cette question porte aussi bien sur la Formulation VI bis du $\$ 3.1$ que sur les différents trpes de la Formulation VII et sur la Formulation VIII au $\S 4$.

II est très probable que la réponse à cette question soit Ifée au cadre heuristique que s'est donné la gramaire genérative. Dans ce cadre, la problématique de l'accuisition est volontairement simplifiae et defigurée : on suppose deux points imrodiats ; dans I'un, le sujet n'a rien intériorisé de sa comptence particuliòre, dans l'autre, il a tout dèveloppé. Ia GU et la MS sont posées carme des pieces essentiellos au mëcanisme de projection permattant l'accomplissement de ce processus instartane ${ }^{33}$. Comme il a té remarqué ci-dessus, on pent cextes, 
Jans ce schena, preterdre a l'enpirisme de la Gl et de la MS; on ne peut en dire autant de leur interprétabilite psychologique : rien, dans ce schema de vecherche ne pentret ni ce verifier ni d'invalider une hyothise sur I'interprëtabilité psychologique de la gl et de la us. En effet, d'une part, ces hpotrièses, dans le meilieur des cas, sont testés dans un donalne très different de celui dans lequel elies sont censés etre interprettables psychologiquenent. D'autre part, dans le caure heuristique fixt, 1 li est par definition impossible a'invalider leur interprét tabilite psychologique : coment peut-an téster que la projection proposée par une formulation particulière sur la Ge et la MS est la correcte

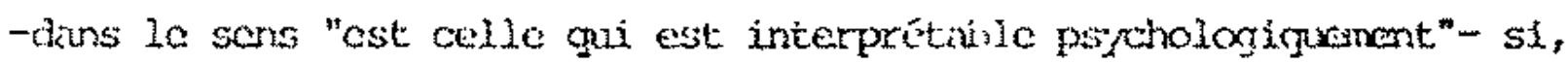
par definition, il n'existe pas de situation effective pernettant d'coserver les donnes susceptibles de la valider ou de l'infimer? En particulier, il est par definition exclv $a^{\mathrm{r}}$ avoir acces aux donnes originales et aux donnes projectives qui, dans les differentes etapes de I'acquisition, seraient susceptibles de tester les mécariszes de projection. II est a remarquer, par ailleurs, que les projections proposés sont peu plausibles. P. ex., au $\S 4$, on a teste des hipothèses empiriques conses etre ausceptibles de projeter la distinction entre cibsenos systematiques et absences accidentelles et de projeter la place de I'accent a partir des tratits phonicues dans la succession des voyelles et des consonnes. Ie

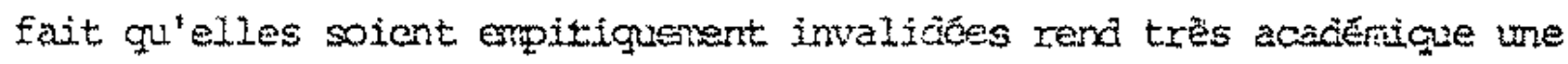
discussion sur leur interprétaibilité psychoiogique. On renarquera, cependant, le faible degré de plausibilité qu' auraity au départ, whe tertative dans ce sens. Si l'on accepte qu'un enfant connait tôt les sequerces ghom niques possibles et que, en revancte, le lexique est justerent tre des zones ce sa compétence qu'il met le plus de temes à acquêrir, on conviendra ga'un mécarisne de projection qui dépend si étroitenent de la présence de tel ou tel signifiant dans le lexique, paraitt particulièxement mal placé parr projeter la distinction entre absences systéntiques et ajonces acicentelles partir des aomés originales efrectivement attestêes vers des donrëes projectives effectivenent attestês. Soit, par ailleurs, cars cette mëne ligne de raisonement, la projection du placoment de l'acoest principal en anglais (ct. § 1.3). Est-il plausible de penser qu'un petit anglophone va projeter la place de l'accent d'un corpus des conness originales ou apparaissent des nots tels que artidisestablishnentarian, 


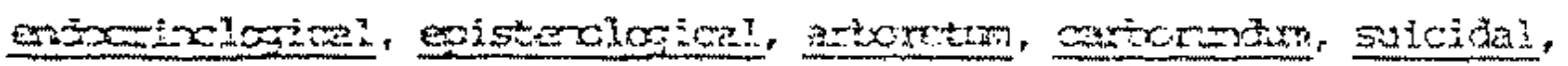

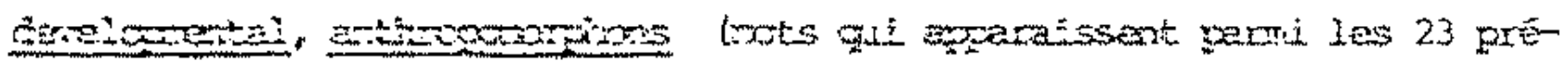

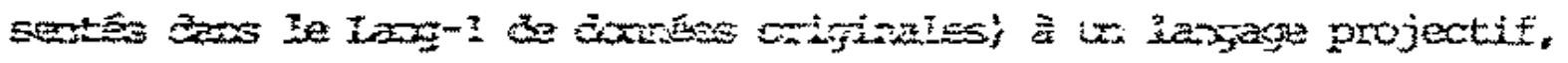

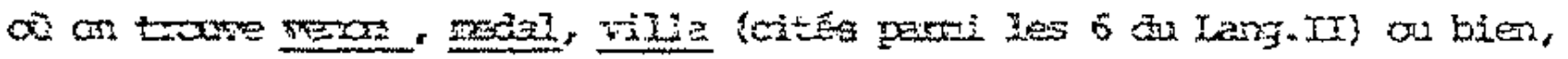

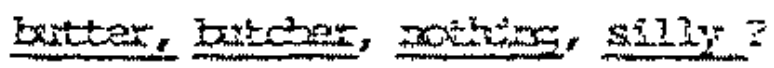

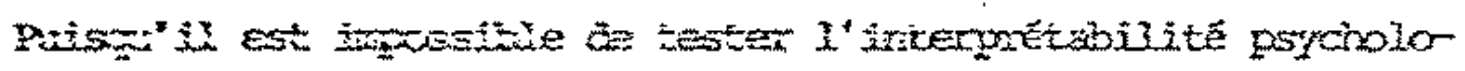

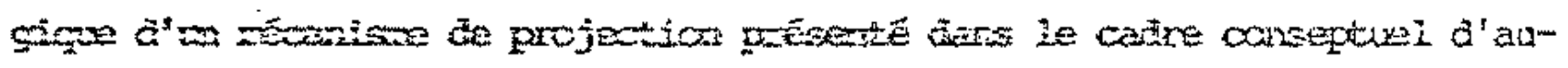

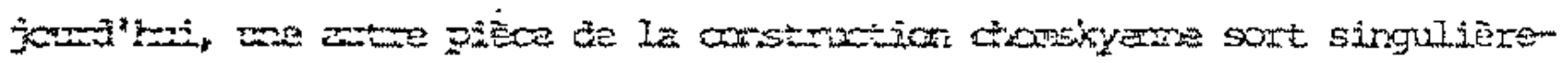

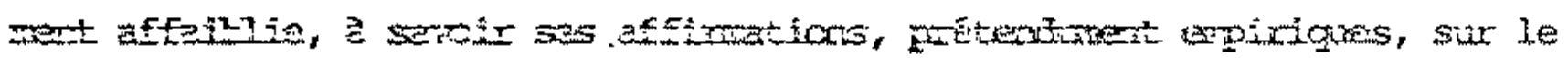

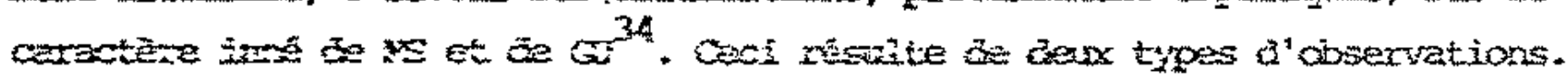

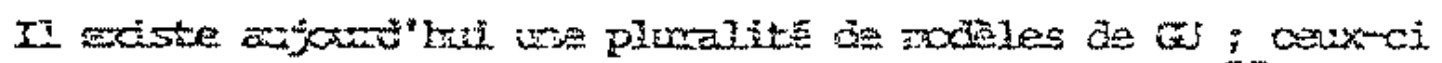

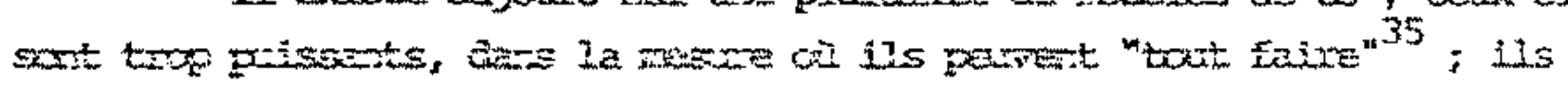

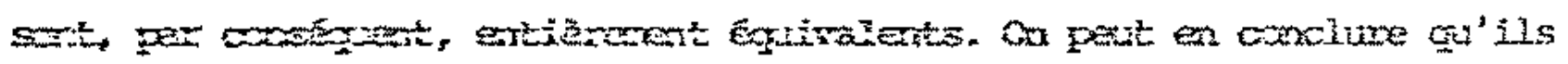

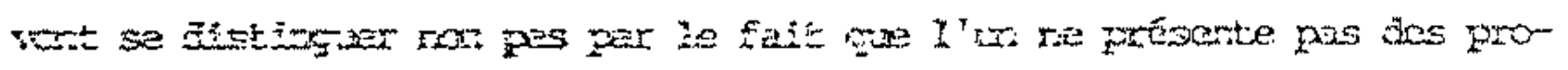

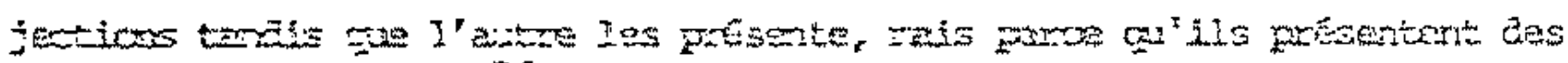

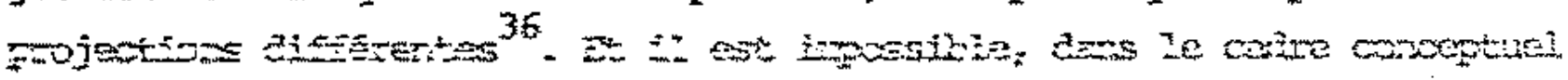

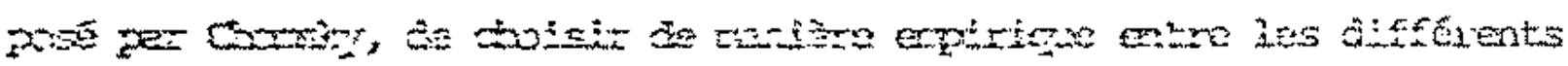

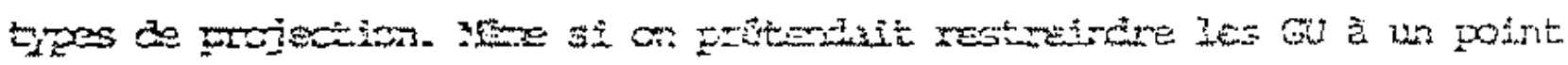

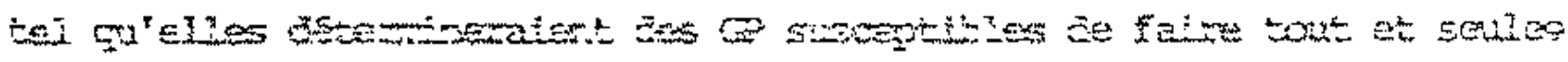

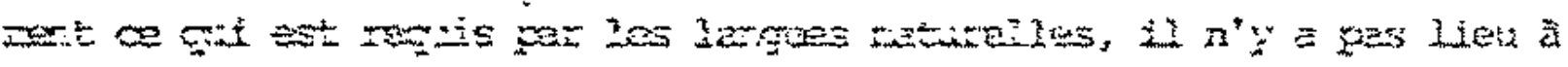

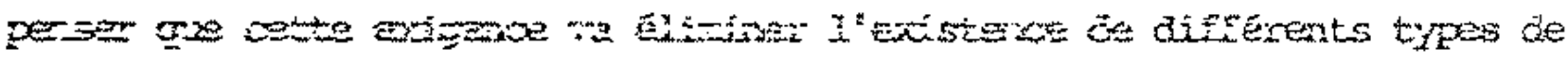

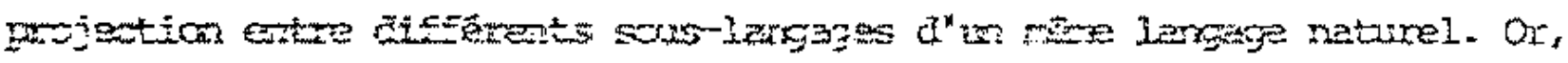

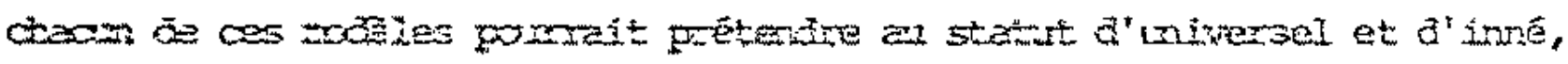

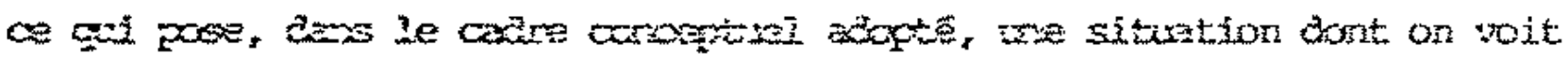
nal 1 issoe Fossibie.

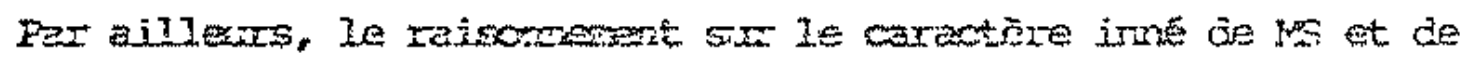

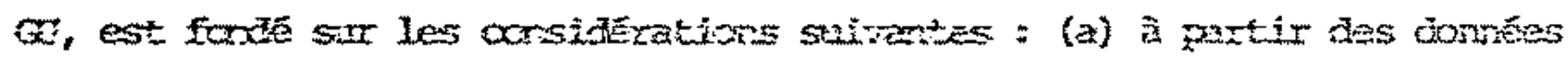

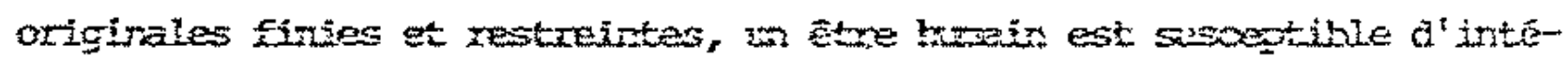

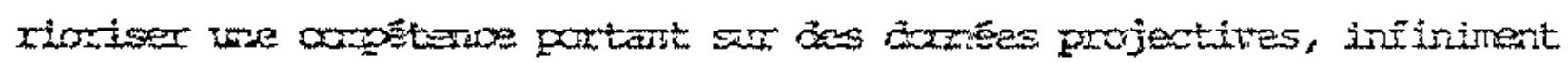

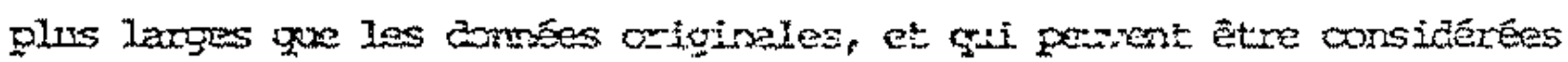

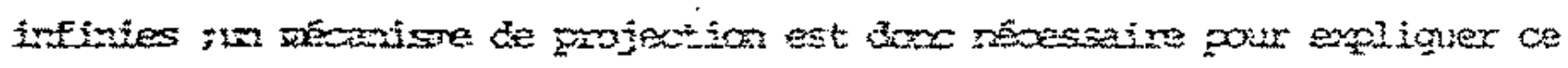

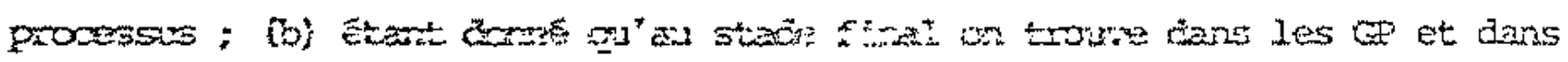

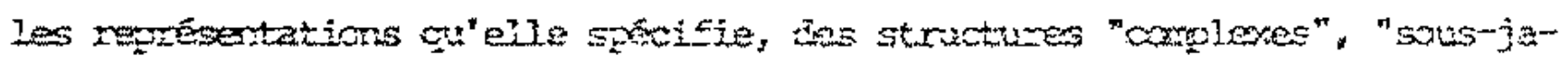

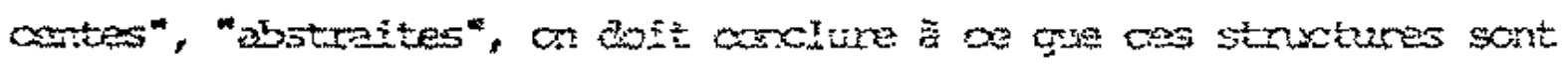

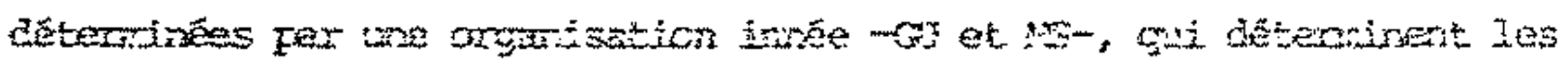


G? - car ces structures, de par Iedr "complexité", "sous-jacense" ou "ahstraction", ne peuvent pas avoir etwé acouises à partir des donrées originales.

Or, ce raisomenent, dans le care conceptuel ou il est presente, aboutit à une proposition ron expirique, car elie ne peut pes Etri Eausse. En effet si l'on re considere que deux points extrêres, ol dans $I^{\prime \prime}$ un on $n^{\dagger}$ a rien interiorise et dans $I^{\top}$ autre tout ; si, dans les connees originales on Ecarte et/ ou on minimise les apports des structures cognitives autres que celles du langage; si on btuile me competence neutre par rapport aux conduites de proiuction et d'interpretaw tion, 11 paraIt Evident que ce qu"on trouve ax stade final et qui apperalt omme etant inwossible d'avoir ete apprehende a partir des donnés originalcs, ne pout pas ne pas avoir ste deteminé par qualque chose, ce Guelgue chose ne powant etre gu'the organisation que 1 'on pose au mornt

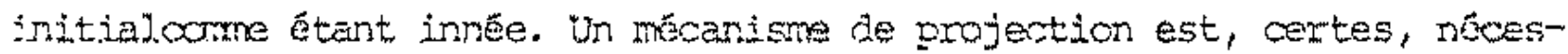
seire, come 11 a ftes souligne par chonsky, pour rendre ompte de l'ac

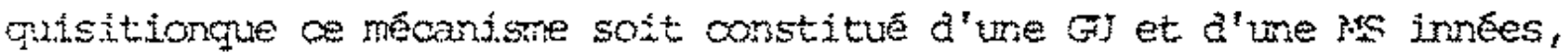
c'est we autre qustion, guestion cul, dans l'etat actuel de la recher che, ne pourra pa etre errpirigue tant qu'elle restera posed dans le catre heuristique ae recherche adopte par chomsing ${ }^{37}$. 
6-1 Dans les $\$ \$ 2,3$ et 4 ont et presentées une pluralité de MS utilistes en gramaire generative. Elles resultent d'une analyse cui ne peut pas être exhaustive de tous les ecrits ingulstiques inspires par la grammaire ghnérative, mais qui se veut représentative d'un certain nombre de textes cles. Certes, l'ambigultê de la notion de simplicité avait dêja ete renarquee, mais la presque aizaine de formulations differentes ou'il est possible d'expliciter à partir des exemples concrets, temoignent des tres grandes faiblesses du cadre conceptuel $\mathrm{d}^{\top}$ ensemble ${ }^{38}$. Et il paraft impossible, dans l'etat actuel des fomulations, de réduire cette trop grande diversite. Il reste cependant a Elucider completement la nature des rapports de la simplicité avec la notion de géneralisation, et cect a l'interieur de la problematique posé par la projection des structures. D'ores et deja il paraitt établi, par la discussion precédente, que la MS utilise dans la Formulation VI n'est pas empirigue et, $\infty$ qul est plus grave, qu'elle hypotheque lourdement le caractère empirique de toute proposition sur la composition d'une GJ. Les Fonmulations VI-bis, VII et VIII posent, en revanche, le probleme autrement, et il $n^{\prime}$ est pas exclu qu'elles puissent tetre rapprochées, a condition de fixer des contraintes aux domées oxiginales et projectives, et aux donnees differentes qui interviennent dans la carparaison des Ge rapprochees dans la Formulation vi-bis.

Le $\S 4$ montre que les MS empiriques explicites quil ont etre proposes sont très faclienent susceptibles d'être invallaées. Le 55 Etablit qu" 1 devient impossible de donner une interprétation psychologique aux MS empirlques susceptibles a'etre formiles dans le cadre actuel. Or, au-dela du constat d'absence de MS explicites, empiriques, et validées, il est possible de formuler l'hypothèse (emplrique) suivante :

Aucune MS ermirique qui. puisse etre propose dans le cadre methodologique actuellement utilise en gramalre gentrative n'est susceptible d'etre conjotntement: (a) explicite ; (b) incorporable à des hypotheses sur I'acruisition du langage theoriquenent coherentes avec les autres hypotheses linguistiques qui portent sur la description de la compeLence et gui sont speifiques de la gramaire génerative; (c) testable dars le domaine psychologlque des conduites atacquisition du langage, si celles-ci sont observêes sans 1" "idealisation" qui les fausse ; (d) vérifiEe dars un certain nonore des cas pour qu'un consensus se dégage à sa 
faveur. Cette hypothèse inplique de faire la prévision forte suivante : mere si une hypothse empirique explicite etalt formulee, testee et validê dans le cadre mëthodologique actuel de la gramaire générative, cette Whothèse sur la us ne serait pas validse par des observations susceptibles d'etre faites dans le domaine de I'acpuisition du langage, st ces abserm vations sont conduites sur des donnëes prochas des conajtions rélies et non sur des domees résutant' d'une "idểisation", heuristiguse au autre. Ceux qui croient que la ou les MS envisagees en gramaire genkrative cht des possibilites d'etre interpretables prychologiquement devraient onsiderer que cette prênision est susceptible d'être irvalidée : il Ienr suffiralt de présenter la ws avec les caracteres précedment signales.

la mor-micite des résultats descriptifs hante la linguistique depuis Iongtemps. On pert accepter, avec Bacil', que I"anrée 1957 a ete une anne capitale et chamiene pour la linguistigue = a"me part, ont êté publiées quelques grandes récapitulations de la linguistique structurale, come le recueil de Joos; d'autre part, les structares Srataxigues de consiny, dont con justifialt en partie les norvelies wopositions parce gu'on atteridat qu'elies soient susceptibles de trancher, de manien sonate, entre solutions concurentes ${ }^{39}$. En effet, le recueil de Ioos finit par la discussion d'ur probleme classique en Iinguistique stnchurale : I'assignation des allophones aux phonisnes cars une larage

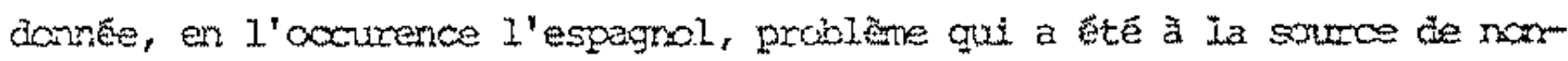
unicites indtiples dars la Inguistique post-sunssurierre et gu, sur

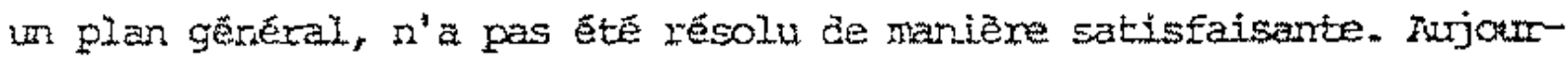
d'hui la gramaire gênerative atant, centes, enrichi la connajissance da

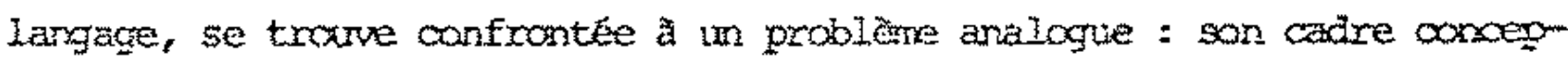
twel n'est pas suffisament riche pour porvoir donnex la possibilite ce choisir de maniere enpirigue entre solutions concurrentes. I' absenoe d'interprétabilite psychologigue des rypotheses entraine la necessité de postar Ier des regles de jeu pernettant de se donner un "critere" pour choisir entre plusieurs hypotheses possibles. Ces regles de jea perrert se multpliex facilement, d"ou l'eclatement en fooles et sous-eooles wivales, et, o qui paraft plus grave, l'absence de commication entre elles.

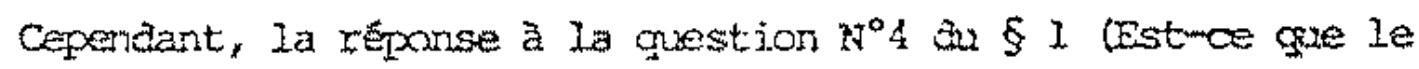
traitenent du problowe de la simplicite a arence par raport a la situm 


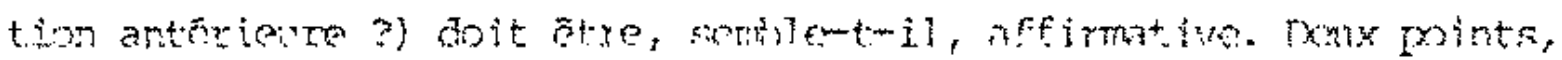

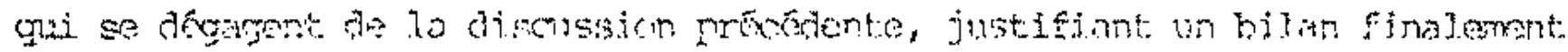

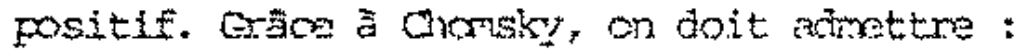

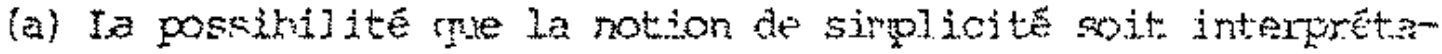
ble psychologiqument.

(b) Ia possibilite que le mécariane de projection quf dott etre inormore axx hyontheses sur le langage compremne urie lis.

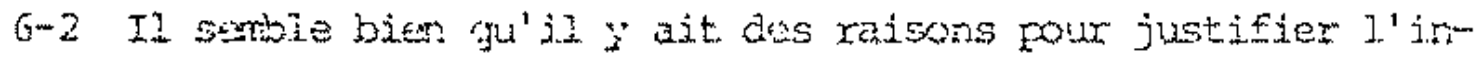

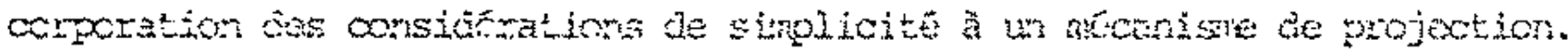

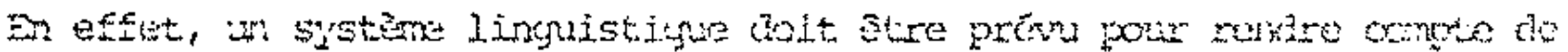

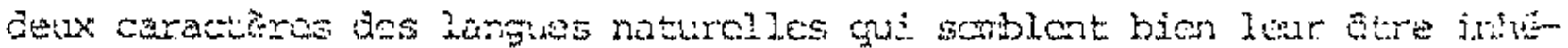
rente :

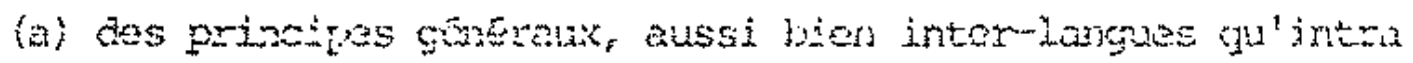

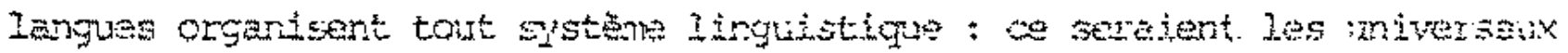

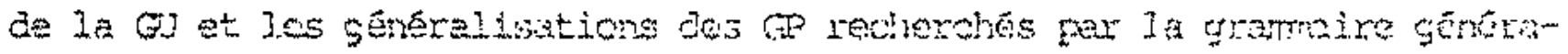
tive :

(b) des cas parbialliers, des excoptions, des zoplications

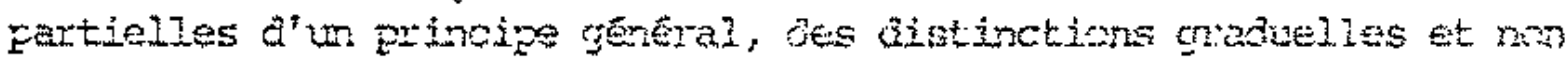
polajres, apparaissent partout dis gu we dosoription est tant soit

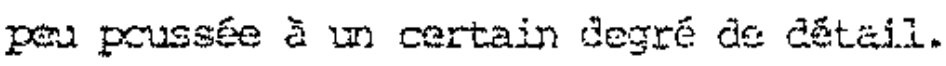

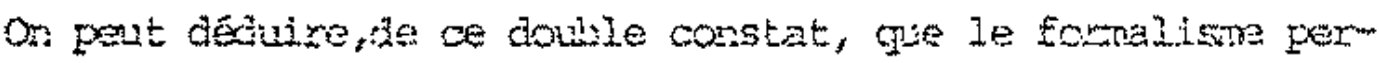

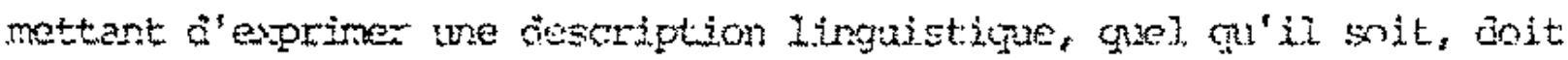

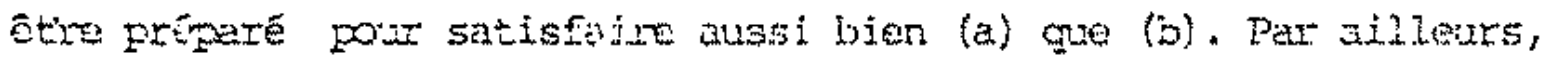

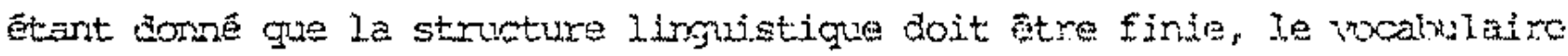

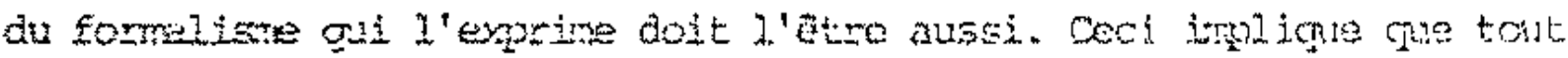

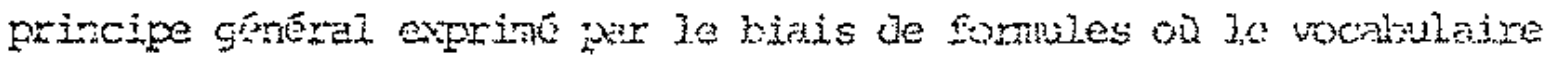

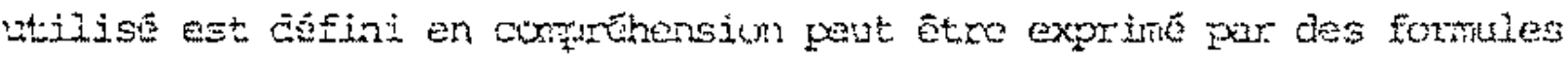

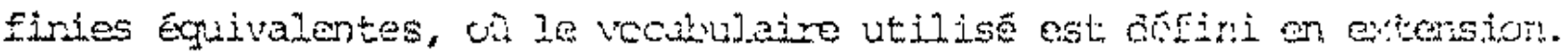

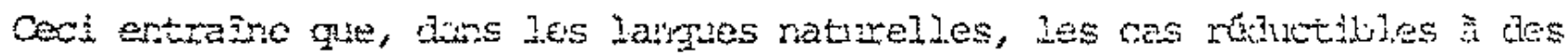

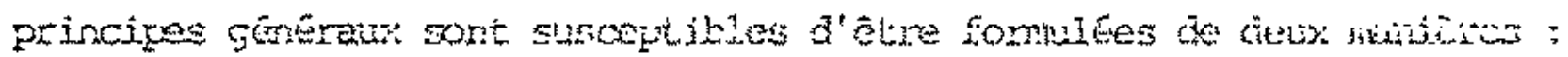

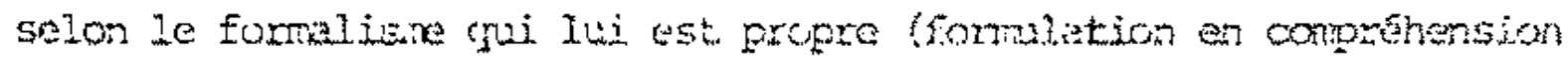

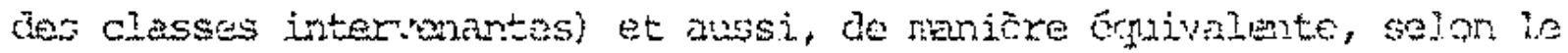

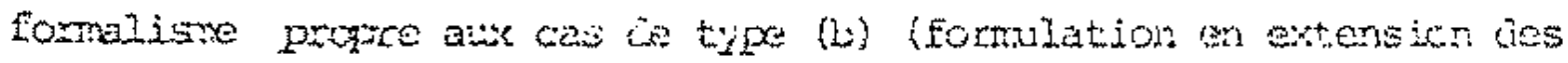




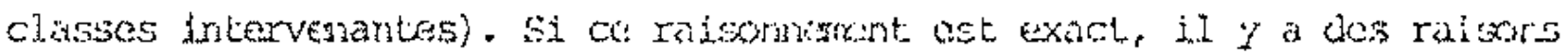

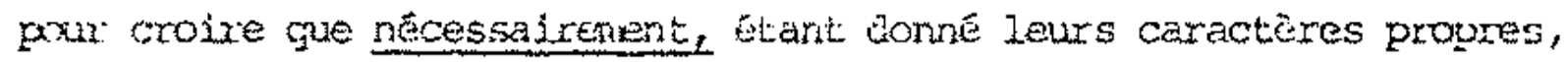
toute formalisation des langues naturelles se doit d'avisager le prom bleme du choix entre altematives tquivalentes : les contitions pour justifier 1' inoorporation neceseare des consioerations fur la simplicild semblont donc exister 40 .

Mais un autre argunent peut encore etre donne pour rendre plausible l'incomporation de considerations de simplicite a la theorie du langage.

Ies donnees extêtieures auxcuelles 1'entant est confronts, colles qui ne reidvent pas do son organisotion montade et qui vamident dx langue ì langue, gu'elles soient rojuctihles ou non a des principes gurranaux, se présertent sous ia forme de cas particuliers: les diffem

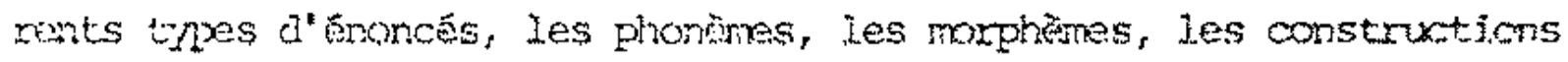
sutariques, les traits semanticues sont, pour ainsi dire, conarstisss

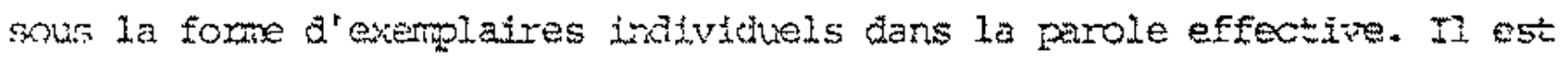

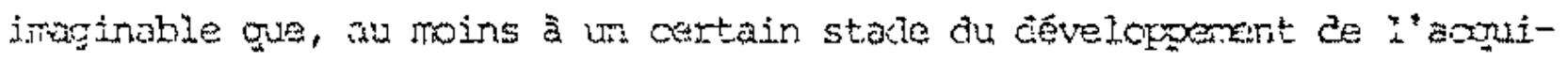
sition, ces exerolaixes soient incorpores en tant que tels ai par le biais d'abstractions cui resterti tris press des cas conmets. Ce seratt par la suite, lonsone le stoknge et l'organisation des cas gertralisobios par le biais ch formalisme adjpte aux cas partiouliers devionnent tro criplexes et couteux en relation au langnge treité, cuse des consiabrations the simplicite obljgeralent a récuganiser lens structuration sous la forme de principas generalux. Dars cette optique, l'acouisition ai languge serait envisagê cume un processus de rerganisation ce structures, conditiomê par un certain ineal ae "uentabilite". Celle-ci resulterait a'we relation ontre le langage que l'enfant doit traiter et plusieurs cotss : cotts de stockage du systerre linguistigue et des instructions d'itilisation de ce systeme et cout de fonctionnement de ces instructions dans la production et 1 'irlexprtation hes froncss ${ }^{41}$. Ces couts senalont precistas par plusieurs mesures, qui ne porteraient pas sin des

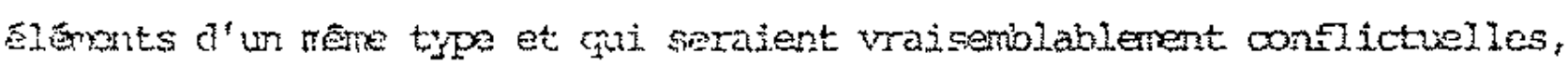
en ce sens gue la retuction a'un tope de cout lc'est-a-dire, la "simpli-

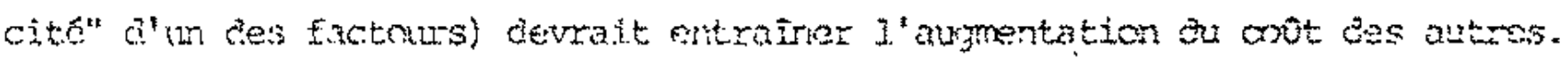

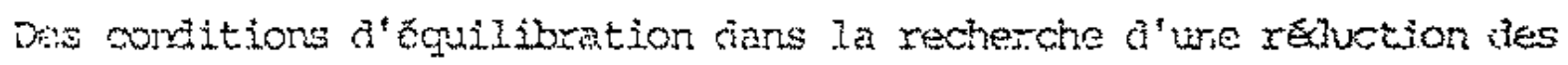


différents types de coût devraient donc exister ; elles ne dêtermineraient pas la simolicite du systeme d'ensemble, mais un equilibre entre plusieurs simplicitès differentes. Chagu rénryunisation des structures, Hitervorint dane les processus d'acquisition, dêtemineralt un effet double : d'une part, diminuer relativement, par rappont au langage traite, les différents couts de ces structures; d'autre part, élargir le langage susceptible d'être traité par la nouvelle réorganisation.

Si la notion de simplicitê Etait interprëtable psychologiquenent et vilidäe, les perspectives qut s'ouviraient seratent interessurtes : il serait possible d'avancer une explication a ce recours à une simolicité formelle dont on a fait usage cans les sciences empitiques. En effet, un enserble de conraissances, sur $n^{\prime}$ irporte guel domajne, doit etre stucturé et codifit pour être stooké à long terme. Ist-ce qu'il est inimaginable de perser gue cette structuration et cette oxlfflcation peuvent être concitionieses par des considerations de coût et d'éruilibration entre couts, considerations qui seraient fomellenent analogues, voire identiques, a selles gui cperent sur le langage humain ? Le langage narouerait ainsi be son empreinte les autres structures oxnitives.

Un long chemin reste à faire avant gue les spéculations précédertes cormencent à se concretiser sous la fome d'hypothèses explicites et testables. Cepenkant, on peut risquel" l'hypothase (toujours enpiriciue) suivante :

Si un podêle gënérall et explicatif ce $1^{\prime a c q u i s i t i o n ~ d u ~ l a n g a g e ~}$ présente conjointerent les caracteristicues suivantes :

(a) il incorpore un modèle explicite de description du systèrie linguistigue ;

(b) il est explicite et testable par rapport ã des conduites d'acxuisition observees dans des corditions effectivement attestées et proches des situations réelles :

(c) il se rêvelle vêrifié dans un narbre suffisant de cas pour qu'un consensus se dégage à sa faveur ; ill doit incorporer une ou piusieurs us empiricues et explicites.

serat-il plus facile de verifier cette hypothese que d'infiner la préédente? 
Des presentztions partielles de ce travail ont etr faites, en janviar-fEvxier 1975, a la Faculte de Psychologie et des scienoes de I'Fucation de I'Universitê de Geneve at au Derpartement des Ietwes Modernes de I'tniversite de Simon Fraser (Turnby, Cancia) et, en mars 1977, at C.I.R.A.L., de I"Universite de Paris III. cette rézaction protite largement des discussions avec A. kutado, E.W. Foberts, J.P. Bayge,

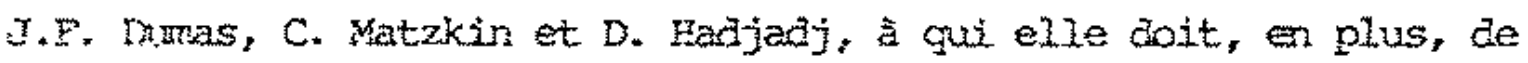
três utiles observations stylistiques. Je remercie R. Zuber de mªir indigue l'existence du travail de soker (ci. 124b).

1 - Non sans gualque axbitraire on peut situer vers 1967 I'introctution et aiffusion on Fance de la grimaire gonerative : après la date de parution de Grumig 43 et avant celle de Rivet Ill.

2 - Les "sciences fomelies" et les "sciences empirigues" de ce travail comesponiert aux "sciences formelles" et au "sciences sur des fatts" ("Factuales", dans 1"originaI) de Bunge (17 p. 30 et suiv.) Far corsequent I'expression "hroothese erpirigue", dins ce travail ne doit pas etre comprise dans ie sens die Eurge (I) p.284). Fur le statut des hypotheses, en particulise dars le domaine Iirguistiugu, of. Eotha 14 p.174-176, Both 13 et Dotha 15. Co twavail a eto conduit inchendement des

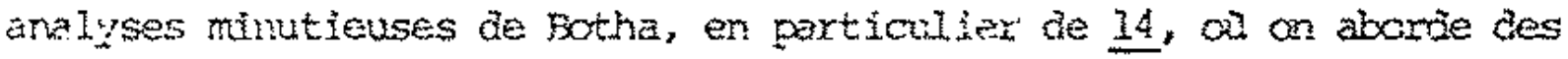
sujets aralogues. Fn lignes generates, on pitsente dans cette contribtion

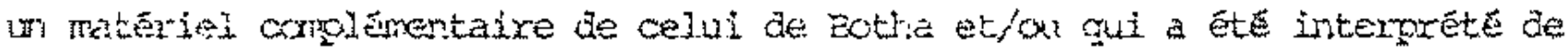
ranjere differente ; an signalera ci-dessous des comergerces et des divergencss.

3 - Sur les notions de modele de boite noire et de boite transparente, cf. Bunge 18 .

4 - Sur la simplicite utilisee pour choisir entre hypothèses empiricues en concurrenoe, of. Bochengki 12 p. 229-230, Copi 33b p. 383-384; Botha 13 p. 47-48 et les remvois qui $y$ sont faits et, touk particuliemenent. Popper 105 p. 128-136 et buge 18 p. 129-184. Celui-ci, d'we part, met en relief les aspects multipies d'an systeme hypothetique gui peuvent etre plus ou moins simples et, d'autre part, montre corrent la similicite favorise seulerent quelques uns des principes gthêraux par raxport aunonels il est possible d'evaluer des hypotheses. Ie rexours la la siripicite dans les 
autres donines (cf. Derving 35 p. 243-245) scimtifigres ost noins

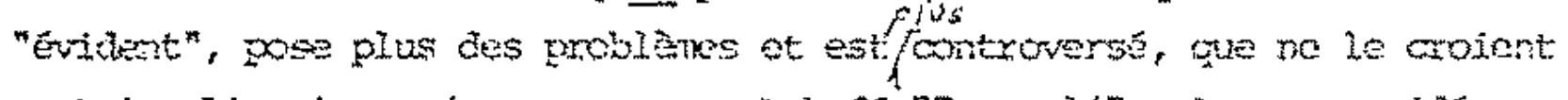

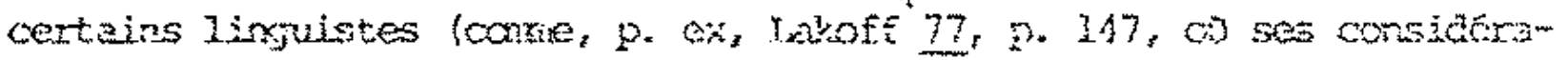

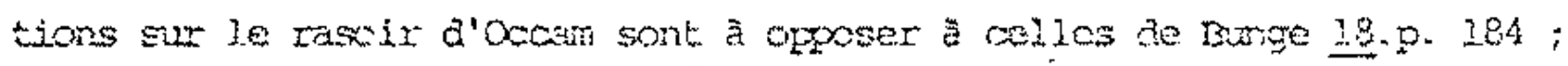

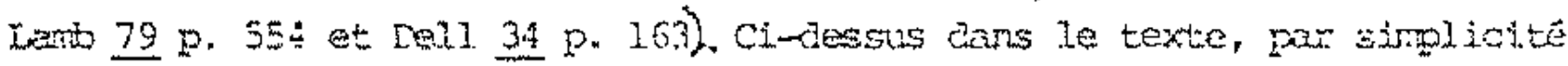
fonnelit, rous compreitons a crue popper clesse parni les conceps the tione et prapatique de la simplicite et non pas la simplicité en tant que orincipe cij favorise la testabilite des rypothöses lof. Popor, ibidam p. $123-134$ et Bunge, ibidgen, P. 138-150).

5 - on constate, chez Stussine (cr. 113 ) I'offort soux matic: en Iaroxt la largue avec la "conscience" diss usagors, te mine que chet. Traibetzkoy (129 en pertie. p. 1-.15, p. 31-41, p. 66-68). Ix notion de

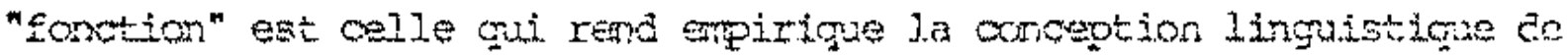

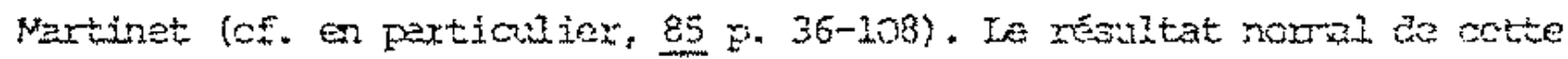

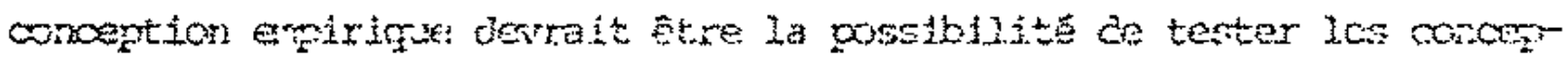

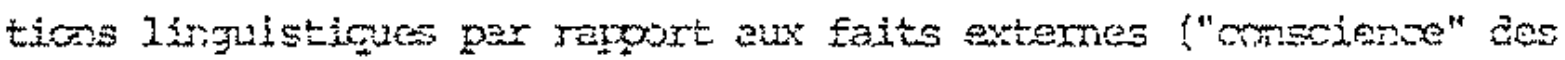

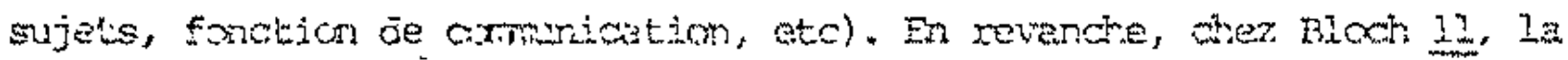

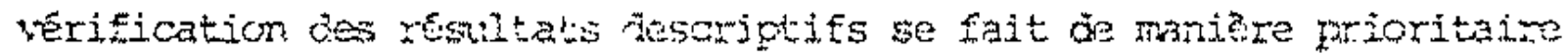
par rapout aux prockes descriptifs.

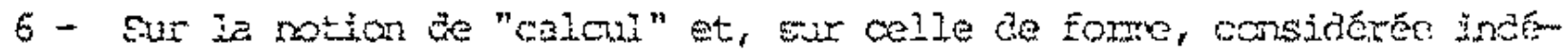

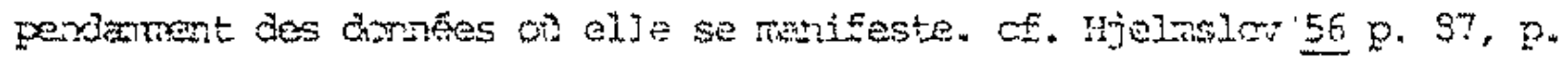
9E-106, p. 114-115, Aess 6 et Bas 7 chap. IV.

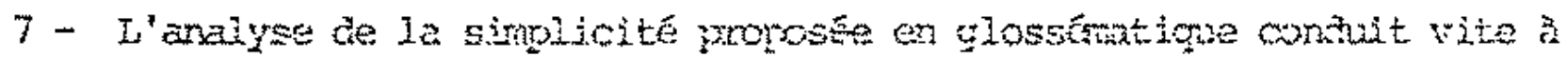

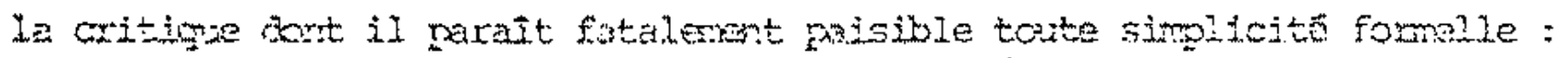

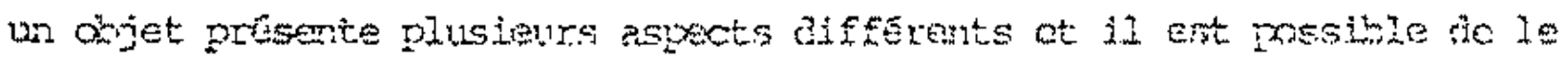

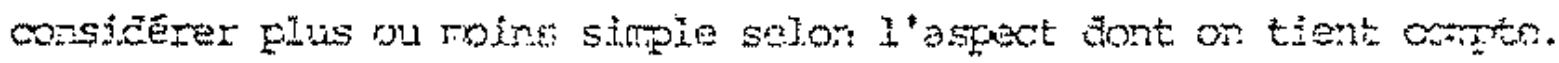

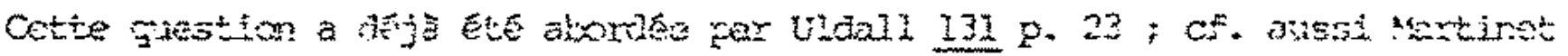

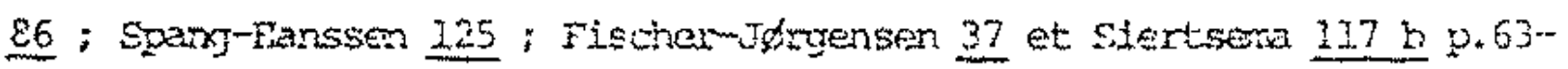

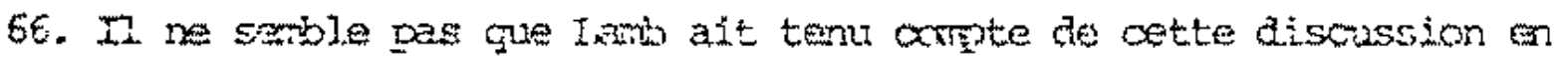

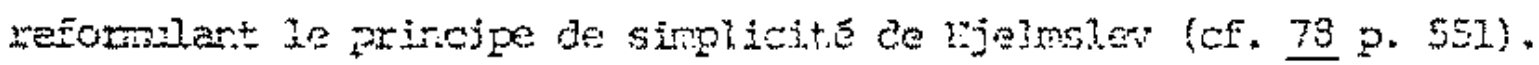

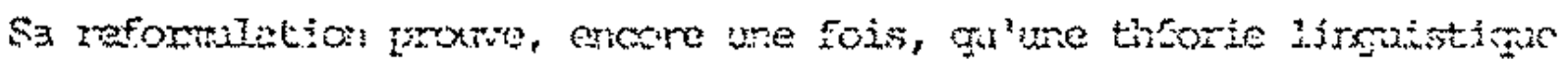




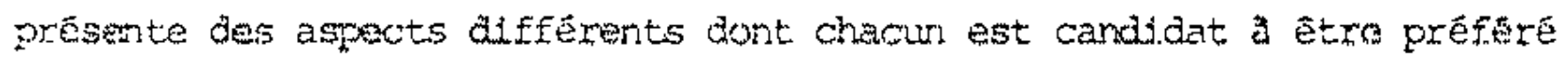
a l' autre dans une hifrarchio d'application d'une simplicité forrello. Ix: criture qu'il propose (cf. 79 p. 554 et suiv.) n'en echappe pas : 1 a note de F. 555 nus inform - sars justification emirigu- gue, pour faire le calcul de la simplicite, il no onsidere que les ligies de son systeme de representation, ce gul Jui pemet d'affimer gue la dastip tion de la fig. 9 est plus simple (et plus gônerale) oue celle de la fig.

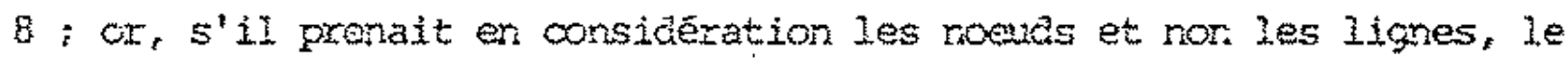
resultat serait le contraire.

8 - CL. Hockett 57 p. $1.10 \mathrm{~m} 111$ crui le presente cepiendant avec beaucoup de precautions (sur l'orientation eroirique de Hockett, ct. en particuler 58). la quete de la symetrie des systemes en linguistique structurale est egalenent en rapport avec une simplicite formelie (cf. Hockett 57 p. 109-120 et Twaddel 130 ).

9 - ce. Jakobson et alii 67 p. 45-47, 66 p. 7, 65 p. 510-522. Il a eté montre que les axantages de la simplicite fomelle sont loin a'etre aussi Evidents que Jakobson le voudrait : la motion "trait aistinctif" presente plusieurs aspects aiffermentes qui deteminent des simplicites differentes (cf. Bess 8) ; par aillturs, la sirplicite formelle proposése n'est pas susceptible de resoudre le problene de la non unicite des descriptions (cf. Bès 7, p. 228-241).

10 - E'etude des simplicites a tef falte come si les textes fomaient: un corpus unioue, sans distinction intemes, wette metrodologie etant justifiête par le type ces citations courament utilisês en Gamaire genfrative : les textes se zenvolent les uns aux autres sans fajre État, en o cul concerne la simplicité, les molifications et/ou des changements de cxitère. Il doit eture cependant possible d'ordonner chrorolcoingenent la pluralite des conceptions sur la simplicite, de telle

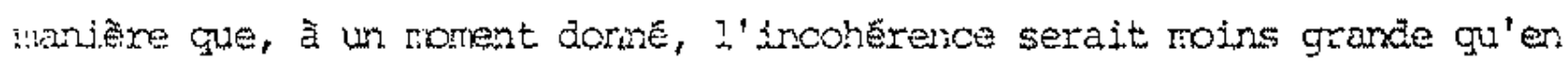
consiobrant spohroniquerent les ecrits ; cet aspect Evolutif n'a pas etế consicisté icí.

11 - Cf. par exenple, la discussion portant aur la differrence notationnelle et/on substanticlie entre sembutiche interpretative et sertantioue 
generative: Chonsky 22, Dostal 108 p. 244-270, Katz 73 p. 397 et suiv. Les participants à cette discassion ne senblent pas avoir teru comple du fait gue I'inoorporation d'une MS empirique, s'appliquant, come toute $\mathrm{Ns}$, aux syntoles utilisés pour noter une stuncture, peut dêterminer gotwe diffisence "notationolle" att des reperoussions expiriques.

12 - Aillars, dans Charsky 20 on retrouve le mere type de raisonnerent, rneme si les expressions "simple" et "sirmilicite" $n$ ' $y$ sont pas

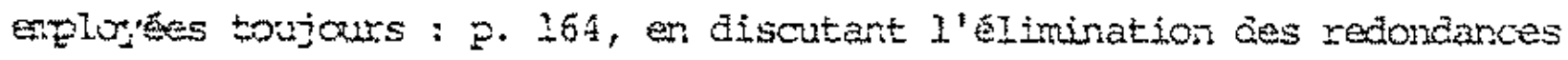
des ertrées lexicales; p. 170-173, polr justifier un certain type de description des Ulemants de Ilexion. Katz I'a utilisê pour justifier le modele de sernantique interpretative (72 p. 250-257, 73 p. 408-4i2); le schére du ratsonnement scus-jacent a été présenté par Eotha 13 p. 29I-292).

13 - La Forrralation I, appliquée au donaine phonique, reapparast ailieurs : cE. dars plile (50 p. 338) la présentation sur le sanihi en saniscrit, et. Iplden (p. 338-340) la premiere partie des discussions sur la spocification des signifiants. Le reme scherna est applique par schane 114, a propos de l'élision des consonnes finales en français, bien que les expressions "simple" et "simplicite" n'y soitent pas utilistees.

I4 - Ia corquaraison de Postal porte sur le modele de la semantique

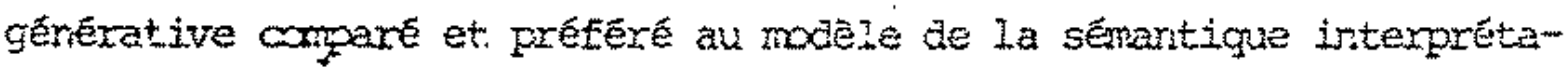
tive. Chez chonsty, un schema analogue -nais non icientìnue- à celui de la Fotralation II est utilise pour justifiex l'élinination de la "redondance" a'un modèle de $\mathrm{g}$ et/ou pour favoriser son "horogenéité" et/ou sa "simplicite" : cf. dans 20 les discussions sur I'blimination des indicateurs de transformations (p. 134-135) et des transfonnations de pemutation (p. 144). On peut comprendre daus ces cas que le nodele de ou parefere corme plus "homoxtène" est, en mêne temps, plus restrictif, les $\&$ gu'il detemine ne devart pas être par conséquent entiè-w retil Ejuivizities a celles de l'autre nulele; en revanche, postal utilise la us cans le cadre du "ceteris paribus". En raison de ces optiques differentes, ni les observations de Eltha 13 p. 309-320, ni la critique. (tacite) de postal par Chonsky (26, p. 125-129) ne sanblent pertirentes. 
15 - La proposition de kalle $(53$ p. 390 ) sur Ies restrictions à introduire à la MS, n'êtant pas arperyée sur la corsidération des donnés extemes, est à classer lans ce schéna, le mene que la discussion de Kucera (76) bien que, au depart, ocininci préserte me ls à classer dans la Formiation WI. Dans la Formidetion III parvent etre clessés

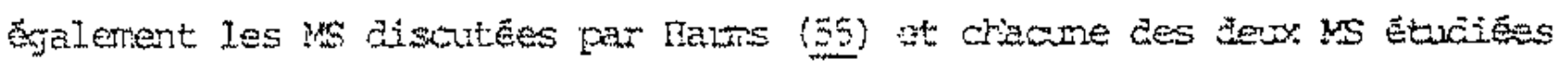
par yen (152 p. 58-68), mass il est dafficine de comprovere 3 partix de

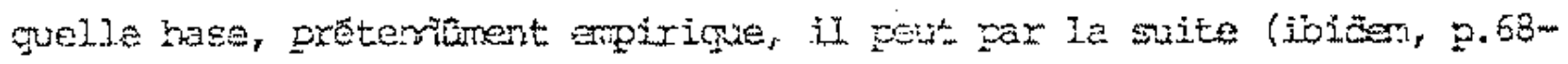

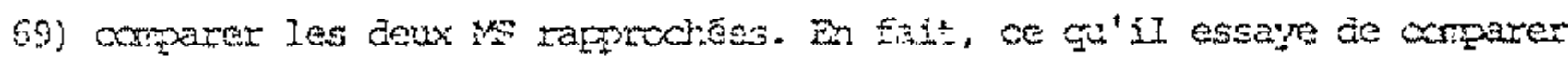
ce sont deux moleles de co.

16 - Il n'est pas surprenant de constater qu'une apprccie formelle de la simplicite pertet à katz (cf. note 17) et à Postal (ct. rote 14) d'aboum

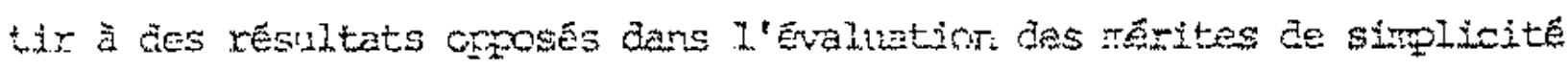
des den: reams roteles fn conourence.

17 - Notia a mis en relisf et la difficulte a deteminer le contemu de It notion de "gtrieralisation significative" et son itrortaros (CF. If chap. III ; 13 p. 276-280). De numse, Fetsers (101 p. La6 et note 12) sculigne one les intuitions zortent zur les exoncés eb ron sur les généraIisations (cf. aussi Kipurshy 74 p. 772 et berting 35 p. 136-isl). Ie

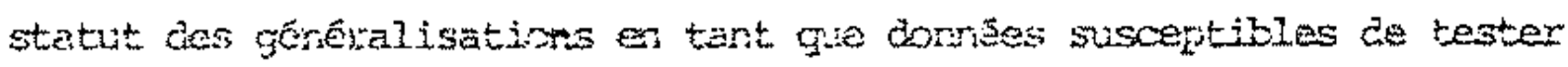

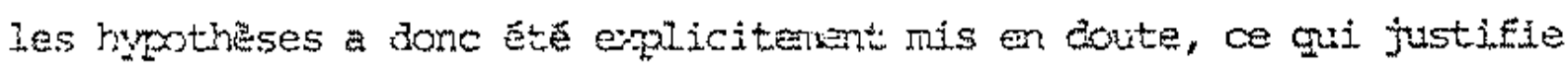

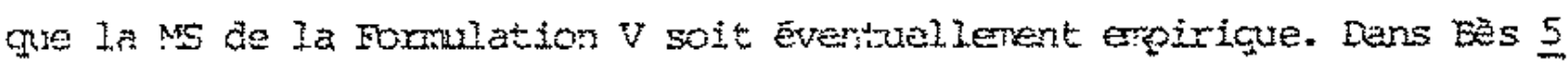
ont the proroses des criterns, fondés sur la notion cle projection, penettant de distinguer les differents tres de generalisation. Ces critêres pouvent constituer des hyothess emirigues et seralent suscoptibles

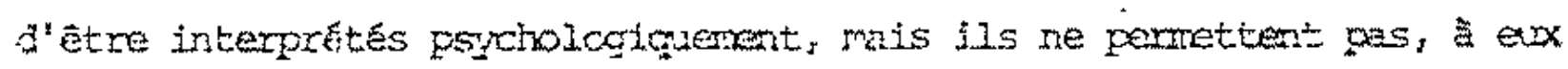
selis, de solutionner lo problene des modiles en conounance et ce pour Tes meres raisons ow'an discutera ci-uessous a propos des projections

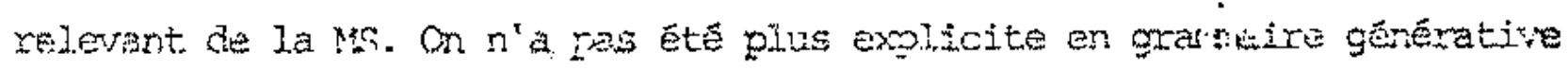

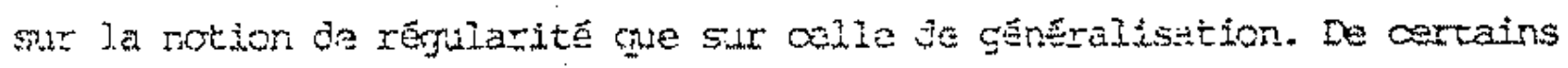

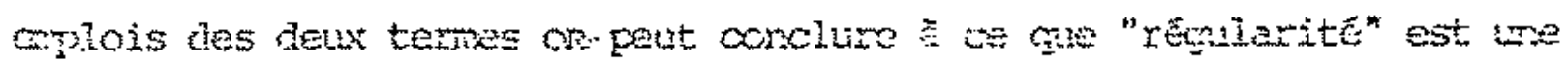




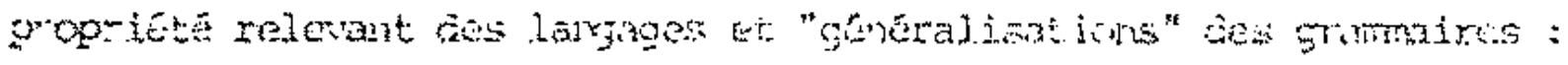

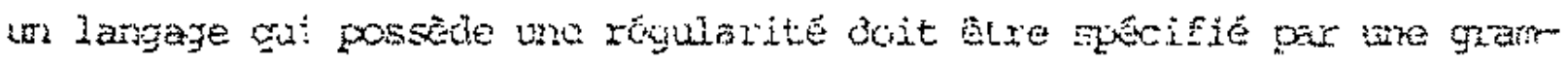
maire qui possçe uno gênêralisation. Je dois à Jean-Franguis Dumas

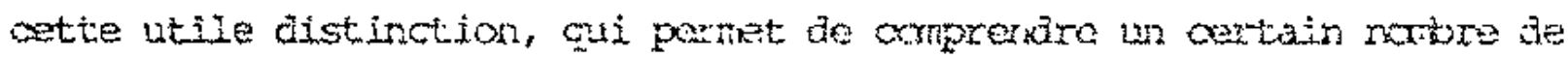
textes portant sux ces questions.

18 - Plusieurs traits des Fomulations IV at $v$ ont Ëté inferés des contextes ou apparaissent les citations. Ie problente du conteru conceptuel

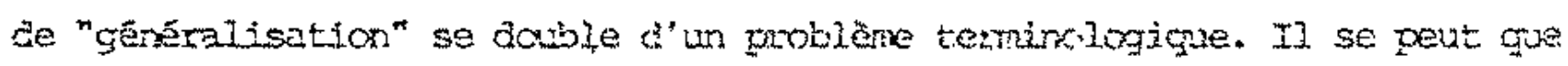

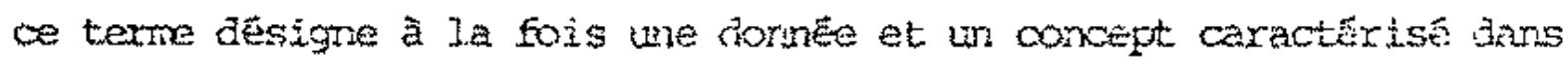
la theorie linguistigue, comie, p. ex., c'est le cas de gramatical, guj

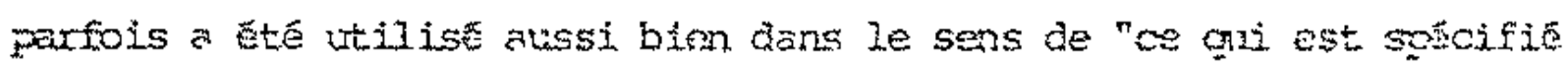
par Ia gramafre" que dans colui de"ce qui est consident come non deviant par un usagex". Nans de cas, la gênerrelisation, rotion oxrop-

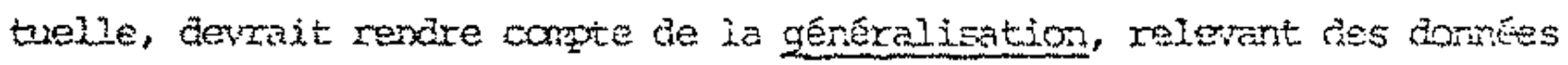
(neis cf. note 17 in fine). Ceci n'est qu' me chestion terminologique ; ce cas de "génétalisation-conceptielie rendant corpte de genteralisationdornée" rentre dans la formilation V.

19 - Si la rotion de classe naturelie en phorologie etait considâte corme le résultat d'ure definition, la las qui y interijent serait une variante de celle de la forrriation $N$ : le texte de lalle $51 p .90$

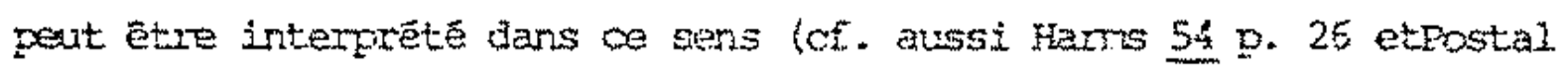
106.p. 73). Er revanche, si elle était considerée come une donnëe (cans la note 22 on trowvera des references attestant l'utilisation do "classe raturelle" cume une donnée) elle apraxtienfrait ì la Formila$\operatorname{tin} v$

20 - On re comaft aucun exorole of la focmilation v axparasse stricterent appliquée, mais elle l'est dans des cas assirilables a cew qui I sert premus : of. Garcia 41, Gaporta et Contrenas 112 p. 10-12, thrmer.

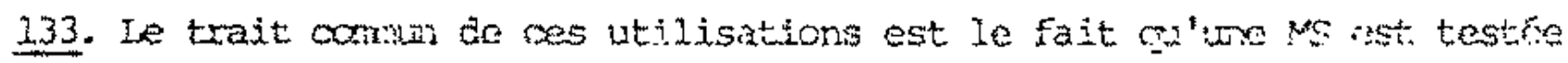

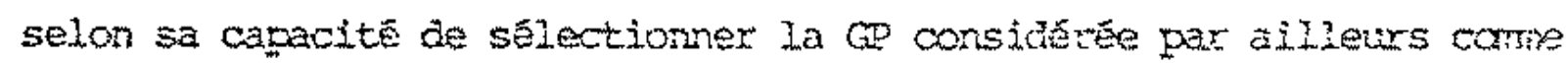
possểant une ackituation externe, celle-ci n'etant fas forctont une "generralisation" mais restant toujours tres fatbienent poecisä. 


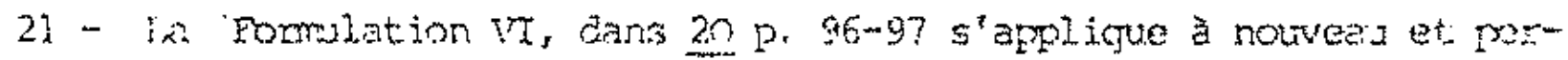
met la justification de Gr-C comportant les conventions (44) et (45).

22 - Ia Fomulation VI $s^{\prime}$ inswert dons un schima de discussion de modèles oli, contrairement aux opinions reoleilites dans la rote 17 , on pose, au depart, gue l'intuition sur la sirilitude de deux faits linguistiques, ou sur leur "complexite", "naturalite"e", etc., peut Etre considérée come one donnête suscoptible de tester des hyothises : ct. Poss 110 p. 229 ; MCarner 89 D. 172, 88 ; Halle 53 p. 183-186; Each 2 p. 76. La Fommation VI est utilisée par Halle 48 et par Halle 50 p. 334-338, Harms 54 P. 7-9 et Deld 34 P. 166-167 pour justifier les traits binaires en phonologie : cf. aussi Hams ibictem p. 110-111 2,a discussion des propo sitions de Mocawley sur les jonctures, Schane 135 p. 34, Contrewas 33 et Bach $2 \mathrm{~h}$ (Ces deux demiers etudies par sober 124b p. 106-107). Sur le plan stntavionie, le schena de la Formulation VI est utilist poux justifier 1'introduction dans la Gu des contraintes "undverselles" qui ne sont pas censes etre respectees dars toutes les GP mats gui, si elles ne l'etaient pas, rendraient les (wip ob elles ne sont pas respectées plus oxplewes : ce. Chomskg 2nc p. 235-236, 24b. p. 75-77, Chonsis et iasnik $32 \mathrm{~b}$. $43 n-437$; un raisonnement analogue dans chonsky 220 p. 176-177.

23 - Les memes observations sont valables pour les exemples rappor tes par Botha 14 p. 69-75, p. 137-139 et le schena de raisonnenent quí S' $y$ applique. Il faut souligner gue, malgré des declarations explicites de Cronsky et de Halle, ce $n^{\prime}$ est pas la pris gut est mise en cause dans les exenples ol se concretise I'utjlisation de la Fonmation VI. P.ex., cuns $32 p .361$, on lit que "en formilant la methathèse de cette manière, nous soulevons un problifme à notre mesure d" "evaluation", et allieurs (ibider f. 400) cue "jusqu'z un certain point, cette mesure donne les rEsultats désirés, mais dans beaucoup des cas, elle bchoue completenent". or. les suites de ces raisonnenents montrent que ce n'est pas la is gui ast rise en cause melle ontinue â etre posece come correcte- mais les

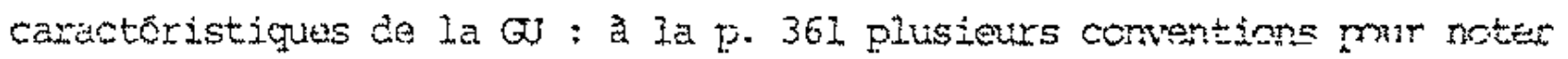
les pethatheses sont rroustes, et, a la sute des exenples de p. 460, la rotten de marque est incorporée. 


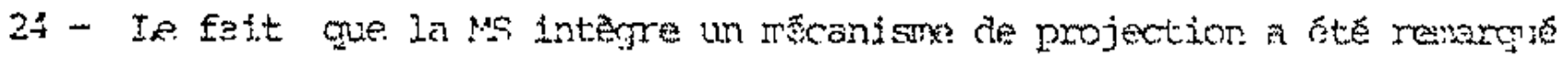
paw perters 101 .

25 - Contrairenant a l'intexpritation de snber (124b p. 106-107), la we de cete illustration re porte nas sur la crmaraism des deix regles de redordance (1) et (2) entre elles. Homis I'excmple proxente ci-ressus, NSQr-A apparalt utilise par Halle 50 p. 340-342 et par Chonsigy 2I p. 64 dans ces exerples qui sont aussi artfficiels que celui rapporte plus hat ; allusion en est falte dans Chonsky et Halle 32 p. 381-382, dans postal 106 p. 164, p. 178 et dans Bach 2 p. 246, sans toutefois que des exemples notveaux y soient présentes. $D^{\prime}$ apres Chonsky (21 p. 64 , et cf. dans le me sers 20 p. 169-170) "Halle montre coment une adhesion cohërente au principe de minimiser les specifications des traits dans la corposante phonologique fournt un fordarient pour la distinction entre les absences ("gap") accidentelles et celles qui ne le sont pas". Or, dans les dellx travaux de Halle cités par Chomiky dars 21, c'est-à-dire dans 45 et. 22 , i) a ette inpossible de retrower des traces de MS G det distinguex entre aosences accióntelies at absences systematicues (cf. en particulier 52, chap. I at II). La MS qui est présentée (pbiàn p. 2930) est a classer dans la Formitation III, les quelnues obsexvations gi y sont faites -p. ex. p. 29- pour essayer de la justizier sont treis loin d'etre suffisantes pour la rendre empirique. Is'affinmation de Cronsiy est bnc excessive parce que, autant qu'on sache, elie est non fondée. Stanley (127) presente un formalisne différent de celui de malie pour rendre corpte des redondances lexicales mais, pour ce faire, iI dolt paresurposer que la MS conduit à des résultats adequats $127 \mathrm{p}, 401$, p. 428, p. 43I), ce qui est faux, les contre-exermies pour le fomaIisme de Halle sont également valables pour celui de Stanley. Bever et Assenbaum (10 p. 3-7) ont utilisse une is dans un cadre analogue à celui de for $A$, nais appligue aux fomants lexicaux. L'exerple qui $y$ est prêsenté est aussì artificiel que ceux utilisés en phonologie ; par ailjeurs, ni la $\mathrm{MS}$ utilise ni la detinition du lexique (vocabulaire des traits utilisés p. ex) ne sont suffisarment explicités pour qu'elles soient testables. 
26 - Four gue I'hypothise ergirigue partant sur p's GJ-3 soit suffisament explicize il favarait indiguer des critères precis pour cerner la notion "Iexigue o'we $\sigma^{\prime \prime}$, selon qu'un itern solt oi non incorpone, il sera pos

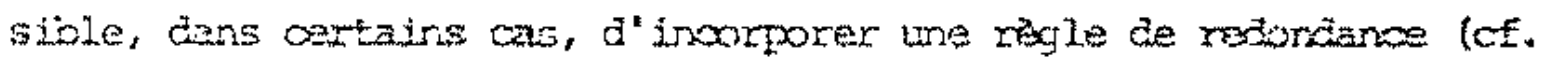

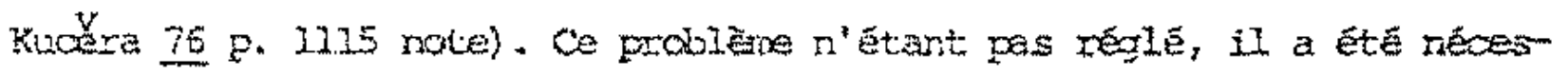
saire de le solutioner de fieniere arbitraire : las dictionaires bilingues françats-anglais (Paris, Larousse, 1960, collection Jupiter) et

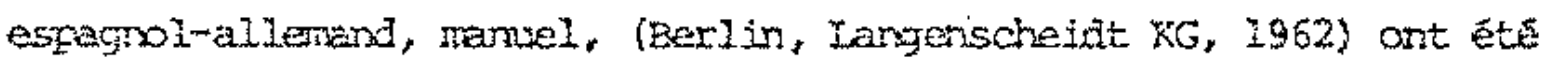
utilises pour illustrer les lexiques du fraricails et de 1 'espagrol; ils présentent l'avantage, étant donnd I'utilisation a laquelle ils sont destisés, de ne pes incorporer trop de régionalisnes, technicisaes, etc.

27 - Le fait de porwolr presenter des contre-exemples et de porroir

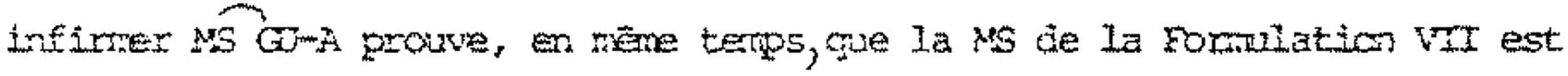
bien enpirique, ontrairement atus conclusions gömerales de Derwing (25 F. 246-247). Te mene Botha (14 p. 237-241) dars ses ormentaires a whe sibuation qui n'est pas entiêrerent identiqua à celle de is $\omega$-A, serble croine ì l'irgositilité de principe de calculer la conplexite dans le

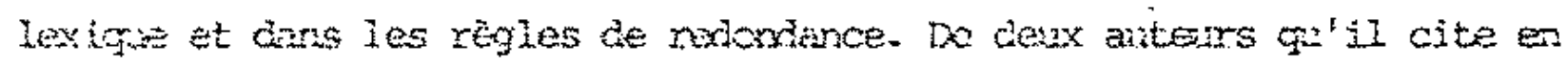

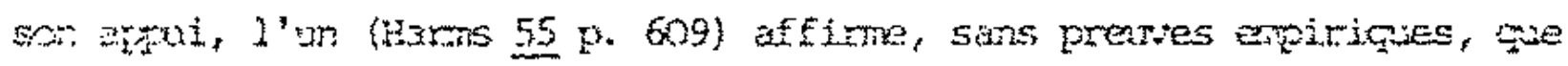

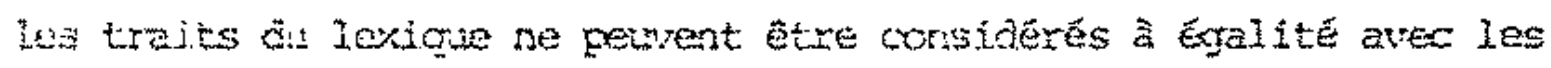

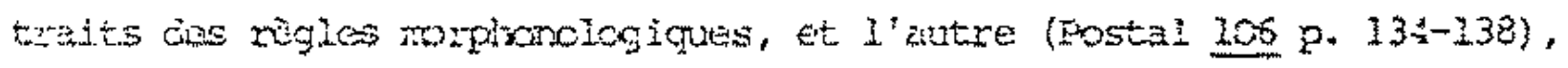

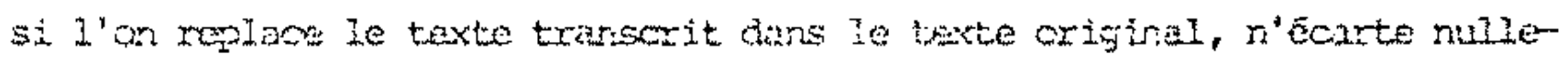
nent la possibilité de mettre en raport la omplexite an lexique ave la

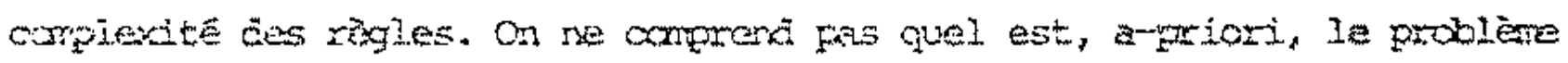
gui se pose par rapport à une lws qui tient ompte des traits distinctifs et cars le lexicue et dans les regles de rodondance : une vague rotion de "piausibilits" or autre ne saurat tetre un obstacie de prinuipe a wa is parfaitement encirigue -bien gu'invalidere portant sur la quantification $a^{*}$ entitss -les traits distinctifs biraires qui sont utilises aussi bien dans les matrices lexicales que dans les negles de redorm dance.

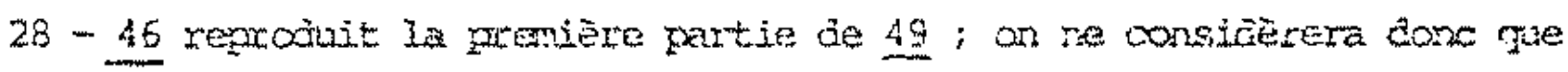

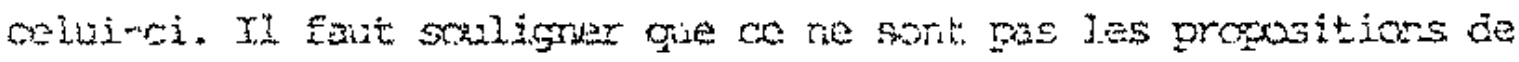


Chaneky et de Balle dans 32 pelles qui sont discutëes, mais la capactét projective attribute a la is at a la Gu dans halle 49 . Dans ce texte, 11 est explicitement indiqus qu'il s'agit de placer l'accent principal dans des nots (cf. p. 2-3); par ailleurs, l'iten (2) presente des mots qui n'ecartent ni prefixes, ni suffixes, ni la composition (p.ex. anti-

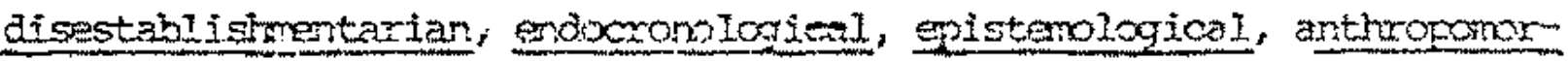
phous) co gui eutorise a en faire eutant dans les contre-exerisles.

29 - On ne dismtera gue les projections du Lang-2 qui sont propres a la Ge plus sitmle et non pas toutos les projentons discuttes dans rielle 49 : il $y$ en a $-p$. ex. la gradiation acoentuelle dans des mots come peisonify qui pervent être projettes par Gril on par cap-2 plus a'autres rêgles susceptibles de s'applinex aussi bien a la sortie de GP-1 que de GP-2. Les transcriptions phonetignes nt la place de l'accert principal pernettant de caracterziser les contre-exumles -dont seulenent des speinens de chague trpe ont ete indigussm ont ette comprimtés au Evary man's Snglish Proxouncing Diationary de Daniel Jones (Ilê ed., Londres et New Yozk, 2956).

30 - Il est sisoue d"avanoer the affimation portant sur $I^{*}$ inexistence te opelque chose dars un donaine aussi vaste que la psycho-linguistique génêrative. Disons qu'll a eté impossible de trower me Ms explicite et erpirique ou une dfscussion aptrofondie et zetaillae de ce probleme dans un certain nombre de travaux représentatifs, aussi bien dans ceux qui ont et inspirés par la gramaire genexative que dans cenu qui ont pris twe position cxitigue. Formis certaines allusions très gênêrales dans Novell1 92 p. 34-36, 94 p. 51-62 (et ct. ausst I'opinion de Moveill cit6e par Derwing 35 P. 1.54n), Fotor 39 p. 11.4, p. 118-120, Praine 16 p. 171-172, p. 120, et cf. ci-dessus $(4-1-3)$ il a eté inpossible de retrouver une us equicite inconporke aux hpotheses sur I'acquisition dans slobin 120 : elle $n^{\prime} y$ apparat pas ni dans la présentation de Globin 119 , ni. dans les contributions de Palento 98, de schelesinger 117, et d'Evin Trits 36; elle n'apparait pas non plus dans kxxley et Ingram 62 (cf. Schelesinger I16, Bellugi 4 Mchler 95, Sinclair 130 , dars plores d'Arcais et Levelt 
38 (cf. Huxley 61, Slobin 122, Rlecel logb) dans Iyons et Wales 82 (Cf.Novelli 93, Klima et Bellugi 75), ni dans smith et Miller 124 (ct. 1'introduction, les commentaires de Slobin a Moveill 54, et Jenkins 70). Une is en tant qu'hypothèse inorroore an modele d'actuisition est abserte aussi des travalux tels que Jakubowicz 68 et Jakuboricz et alis 69 (cf. aussikehler 95 et Mehler et Noizet 96b) qui presentent un aperch genexal des apoorts de la gramaire generative en psycholinguistique; elle n'apparait pas non plus dans des articles qui, inoorporés à des recueils, ont fait le point des problènes dans ce donaine (ct. knteill gl, Slobin 121, Carnoll et wales 19) et dans la contribution de Bever (9). qui represente un toumant important en psycholingulstioùe générative. Elle est absente du recueil récent sur les rapports de la theorie lin guistigne avec lâ réalité psychologique (cf. Halle et alit 53 b).

31 - Le probleme se complique du fait que la notion de generalisatira peut -et vraisemblablement, doit-se préciser en fonction de la possibi- . Iite de projection (cf. notes 17,28 et 36). Par consecquent, il faut non sculement choisir entre differerts trpes de projection, caracterists par les donnes originales et projuctives, nais, aussi, choisir entre projections obtenues par MS $\widetilde{U}$ et projections obtenues par $\sigma U$ sans intervention de MS (tout au moins dans les formulations existantes, cax il est probable que des considerations de simplicite relative doivent intervenir toujours; cf. ci-dessous), Par ailleurs, si I'on adrettait que tous les trpes de projection devalent etre aomis, il faudrait preciser guelies portions de chaque gramaire devraient être traltes par chaque tipe de projection.

32 - La distinction entre hypothesse mpirique et hyothèse mirique interprétable psychologiquenxant correspond à celle présentse par Botha (14 p. 170-376) entre theorie linguisticue non mentaliste et theorie Linguistique mentaliste, celle-ci se presentant conme la description d'un mécanisme mental ; cf, dans le mêne sens, peters 101 p. 174-175. Wais, contrairentent a Botha et peters, il parait irpossibie que i'cin n'exige pas de la théorie linguistigue d'être interprétable psychologiquement. Si lion $n$ 'exige que la linguistique soit empirique et si le raisonnement qui suit est valable, elle ne sera plus qu'une discipline 
Eartiellement empirique, avec des manges de varlation trop grandes, gui devrort etre coniblées par des décisions non-empiriques.

33 - ct. Chansicy 20 p. 32-37 et note 19 p. 202, Chonsky et Halle 32 p. 331, Chansky 25 p. 15, p. 119-122, p. 148 et note de p. 245.

34- cf. Chansig 24 et 25, Les affimations sur le caractere inne de wS et de $Q$ sont faussement empirigues dans le chanp d'observation des donnes Iinguistigues; elles ne le seraient pas, en droit, si l'on se donnait la biologie come domaine d'observation susceptible de tester I'hpothèse innêtste lce point m'a eté éclairé par une discussion avec J.F. Demas, qui, cepencant, envisage I'hypothè inreiste dans une perspective differente).

35 - Sur le trop grand porwir des modeles de G, cf. Peters et Ritcine 102, 103 et 104, Jevelt 81 v. II, chap. 5, Pach 2 chap. 9 a 11.

36 - Cr. darns Bes 5 des exenples de non unicite des projections deter inines par les generalisations significatives; la variation des resultats est deteminee par I'utilisation de modeles de QI diffrenentes et/ as per la consideration de corpus diffterents.

37 - Dans Bès 5 ce raisonnement a eté discute avec plus de dêtail, en particulier, en contestant la neutralite de la compotence par rapport a Ia perfonance. La non considération des apports des structures connitives antres que celles du langage dans le processus d'acquisition apparrait dans Chonsigy 23, 20 chap. I, cf. Ievelt 81 v. 3 p. 144-156.

38 - I'ambigurté de la notion de simplicite en gramalre gênerative avait ete, en termes gênéraux, renarguée, elle est implicitement signalé dans la réponse de Householder 59 et, expliditement, dans Garcia 41, Matrews 87 p. 276, Botha 13 p. 291, Momin 97 p. 219-221; cf. aussi pottier 109 p. Il et hagege 44 p. 120 . Les huit formulations ici explicitées dontent cecerdart une mesure plus exacte de $I^{\dagger}$ acuit du probleme. Elles $n^{\prime}$ Epuisent 
coperdant pas ies possibilitês existantes, au moins trois autres simplicités powrat s'y ajouter : (1) la simplicitê a laquelle fait allusion

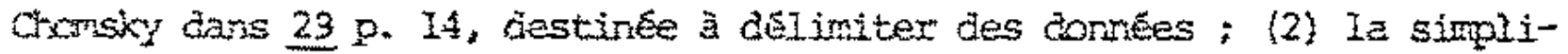
cite portant sur les aerivations, suggertê par chonsky et biale (32 p. 63) ; (3) Ia simplicite de Kiparsky (7) , dont ia validation enpirigue devrait se faire dans des donares läillectoicgie, diachronie) qui debortent celui de la inguistiga descriptive. Il est facile de detuire de cette diversite juson'ì qua point ont ête peu fnuctueuses les discussions sur la sinplicité en grameire gênérative, developoses sans tenir contre de 1 a riche variete des HS (ct. Chmsicy 27, Cromsky et Ialde 31, Halle 47

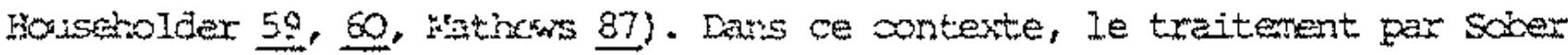
(124b chap. 3) de la sirnjlicite en gramaire gênérative compe un

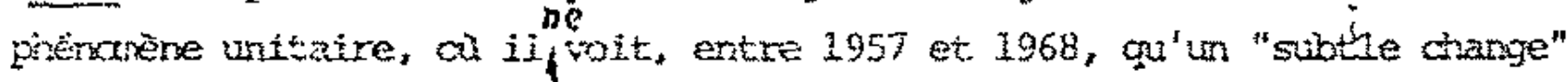
(ibicen $p$. 69) et son essai de I'expinter gar la notion gentrale de sim plicite gu'iI propose, sentulent vouks a l'echer; les illustrations gu'il presente relevent, en efret, de trois formalations differentes : Fonnula-

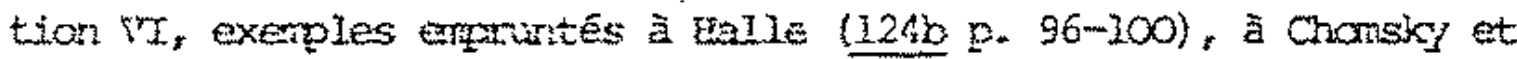

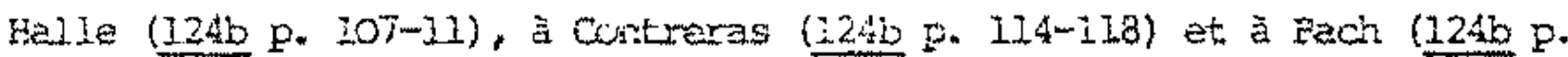
118-120j; Fommlation $V_{r}$ axemles sur les classes natrrelies en phonologie,

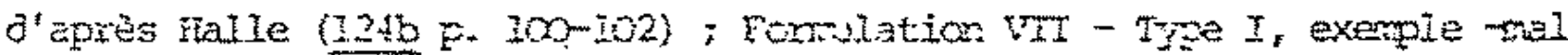

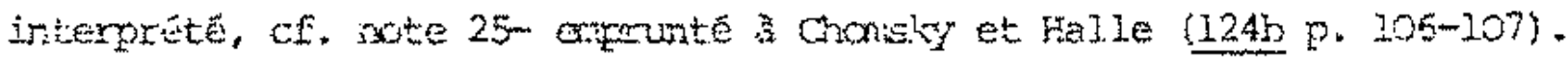
Il fadt Etet, en plus, de la simpicite coi relève de la Fonalation I $\{1240$ p. $94-95, p, 102-103\}$.

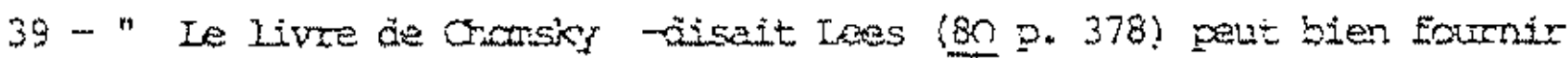
I'op portunite a'swiquer des nesures de simplicite explicites pow

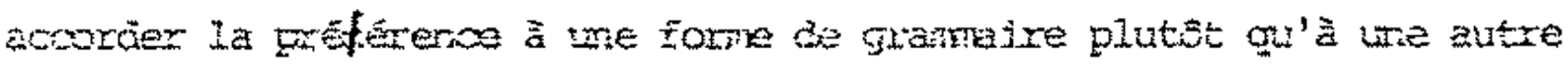

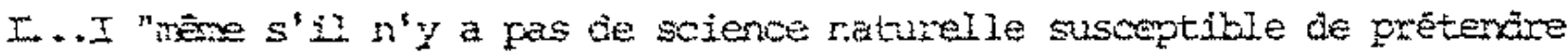
à une procéture d'evaluation vraie, chorsixy Goit que la linguistique

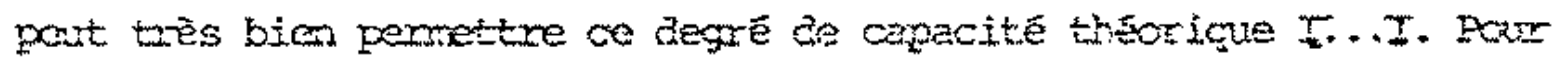
rejetter la maveise alternative d'une paire de theories proporêtes, le mieux que le savant dex scierces naturalles perti faire est de proposer un "experinent coucial"; nals les linguistas peuvent faire un pas au-deia

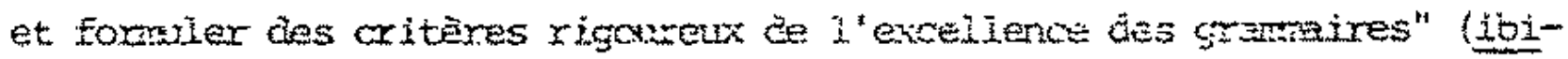
ders D. $380-381$ ) . 
40 - Ces considerations de sinplicite n'aurajent pas le caractêre supplétif que bothe attringe a la sirulicite chansigenne, son abjectif n'etant pas de pallier une insuffisanoe dans la fomulation de la oJ (14 p. 179180), Contrairenat a Garcia (11 p. 869), des consichnations de simplicité ne sont pas methodologicuenent superfues : "la simplicité -dit-elleest innécessaire lorsqu'elle est en accorca avec les intuitions des usagers et sans pertinence lorsqu' elle les contremit". Or, les intuitions sur le langage sont des donnes et la simplicite une hypothèse empirique

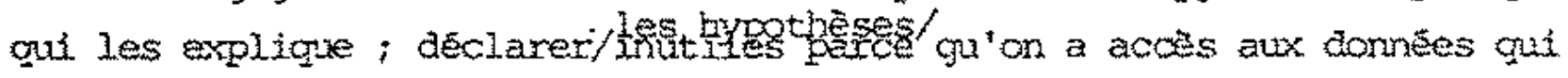
les testent, reviendrat à dêlarer la physique inutile parce qu'on connait les mouverents des astres ou la science inutile parce gi'on pout observer 1'univers.

41 - Ces suggestions, très largement à l'etat de programe de travail supposent une vision des rapports de la conpetence avec la perforanance differente de celle qui est habituelle ; CF. Eess 5 , ol on introduit le distinction, pani les instructions d'utilisation, entre les règles (firies) et les prockures (infinies). Le systeme linguistique sousjacent. les règles et les procedures se conditionnent réciproculument et constituent un triplet d'utilisation, lequel doit être vraisemblabiement different pour auditeur et locuteur. Cette notion d"Éguilibration entre simplicites conflictives s'apparente à celle d'Économie, de Martinet, utilisée avec succès dans le cacre diachronique (cf. Martinet 83 chap. 4 et 84 chap. 6 ; cf. aussi Derwing 35 p. 344 ). 
1- BACH,E. "Linguistique structurelle et philosophie des sciences", in Diogine 51 (1965), p.117-136.

2- BACH,E. Syntactic theory. New York..., Holt, Pinehart and Winston, 1974.

2b-BACA,E. "Two Proposals conceming the simplicity metric in phanologs", in Qlossa, 2 (1969), p. 128-149.

3- BACH,E. et R.T.HARM (eds). Universals in Iinguistic Theory. New vork..., Holt, Finehart and Winston, 1958.

4- BEIEUCI,U. "Sirplification in Children's Language", in 62 p.95-119.

5- BES,C.C."Les conduites simulées; fonctions de cormunication et de représentation", in J.Piaget, J.P.Bronckart et P. Mounoud (Dir.), Ia Psychologie. Paris, Gallimard, collection Ia Plérade, sous presse.

6- BES,C.C. "Forme et substance", in A. Martinet (Dir.), La Linguistique; guide alphabétique.Paris, Denoël-Contrier, 1969, p.117-124.

7- BES,C.C.Identités et différences dars les unités de ceuxième articulation. These. Giversite Rene Descartes-Paris V,1972.

8- BES,C.C. "Trait distinctif", in Word, 23 (1967), p.37-46.

9- BEVER,T.C!The comitive basis for linguistic structures", in J.R.Hayes (EJ.) Cognition and the Development of Language. New York, Wiley, 1970,p.279362 .

10-BEVER,T.C. et P.S. ROSENBAUM."SOre Lexical Stmatures and Their empirical Vajidity", in 64 p.3-19.

11-BLOCH,B. "A Set of Postulates for Phonemic Analysis", in Ianguage, 24 (1948), p. $3-46$.

12-BOCHENSKI, I.M. Los métodios actuales del pensiamento. Traduction de R. Drudis Baldrich.Nadrid, Esiciones Rialp,1957.

13-BOTHA, R.B. The Justification of Linquistic Hypotheses. The Hague..., Mouton, 1973.

14-BOTHA, R.B. Methodological Aspects of Transfornational Generative Phonology. The Hague.... Mouton, 1971 .

15-BOFHA, R.B. The Methodological Status of Cramatical Argumentation. The Hague..., Mouton, 1970 .

16-BRAJNE, M.D.S. "On Tho Types of Models of the Intemalization of Cramams", in 120 p.153-186.

17-BUNGE, M. La investigacion cientifica. Traduction de M.Sacristan. Barcelona, Aries, 1969.

18-BUNGE, M. Teoría y realidad. Traduction de J.L.García Molina et J.Senqere. Earcelona, Arie1,1972.

19-CAMPRELL, $R$. et R.WAIES. "The Study of Language Acquisition", in J.Lyons (Ed.), New Horizons in Linguistics. Penguin Books, 1970, p. 242-260.

20-CHOMBK, ,N.Aspects of the Theory of Syntax. Cambridge, Mass., The M.I.T. Press, 1965.

200. CHONSK., N. Aspects de la théorie syritaxique. Traduction de I.-C.Milner.Paris, Editions di seul1,1971.

20c-CHOMSKY,N. "Conditions on Transfornations", in S. Anderson et P.Kiparsky (Eds, ), A Eestschrift for Morris Halle. New vork, Holt, Rinehart and Winston, $1973,0.232-286$. 
21- Madrow, N. "Current Issues in Linguistio Theory", in 40 p.50-118.

22- CHCHKN,N. "Deep structure, Surface structure, and Sementic Interpretation", in 28 p. $62-119$.

220-CHCurSK, N. "Etats initiaux et Etats stationnaires; I'approche linguistique", in M.Piattelli-Pazmarini (Ed.), Thêries du largage wheories de l'apprentissaze.Paris, Seui1, 1979,p.169-17\%.

23- CHOAEK, N. "Explanatory Models in Linguistics", in E.Nagel, P. Suppes et A. Tarski (Eds.), Logic , Yethodology and Philosophy of Science. Stanford, Cal., Stanford Untversity Press, p.528-550.

24- CHOHSK.,N. Le Jengage et la pensée. Traduction de L.-J.Calvet.Paris, Payot,s.d.

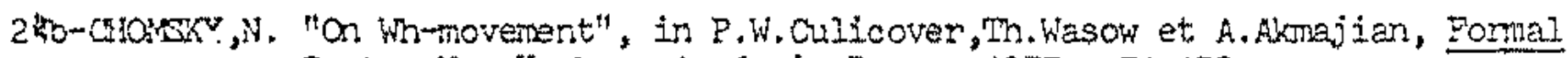
Syntax. New York..., Academic Press, 1977,p.71-132.

25- Crolszk, N. Reflections on language. New "ork, Pantheon Books, 1975.

26- CKousky, N. "Sone Empirical issues in the Theory of Treansformational Cramar", in 28 p. $120-202$.

27- CHOMGKr, N. "Some Ceneral Properties of Phonological Fules", in Language, 43 (1967), p. 102-128.

28- CHows. ,N. Studies on Semantics in Generative Cramar. The Hague..., Mouton, 1972.

29- GONSK, N. Syntactic Structures. The Hague, Mouton, 1957.

29b-CicisK, N. Stmuctures syntaxiques Traduction de Michel Braudeau.Paris, Editions du Seuil, 1969 .

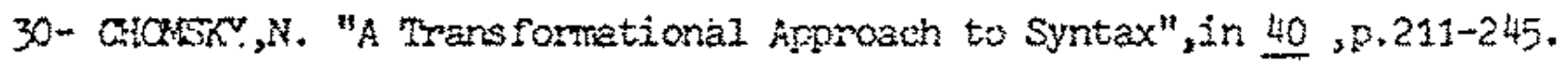

31- CHOHSX, N. et M.HALIE."Sone Controversial nuestions in Phonological Theory", in Journel of Linguistics, 1 (1965), p.97-138.

32- Gasir, N. et M.HALE. The sound Patterm of English. New vork, Harper and Row, 1968.

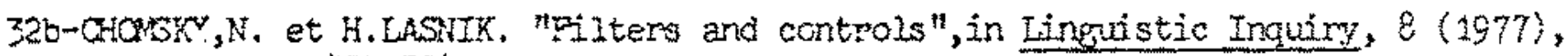
p. 425-504.

33- Caripgras,H. "Simplicjty, Descriptive Adequacy, and Binary features", in Language, 45 (1),1969, p.1-8.

350-COPT, I.M. Introduchion a la logica. Traduccion de N.Mjguez. Buenos Aires, Eudeba, 1962.

34- IEI, P. Les règles et les Sors. Paris, hemann, 1973.

35- DERFINC,B.L. Trans formational Crarmar as a theory of Language Acquisition. Cambridge, at the oniversity Press, 1976.

35b-DUdAS, J. -F. Iá Exammire générative et le problème de I'innéisme. Thèse. Universite de clemont II, 1970 .

36- ERVM-IRIPY, s. "hi Overview of theories of framatical Development", in 120 p.189-212.

37 FISCER-JORCENSEN, E. "Remarques sur le principe de l'anaivse phonemique", in Recherches stmucturales, TCLC, V (1949), p.214-234. 


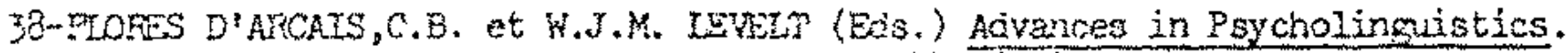
Ansterian... Horti-Hollatio, 1970 .

39-20DOK, J.A. "How to leam to talk: Sone Simple Ways", in $124, p .105-128$.

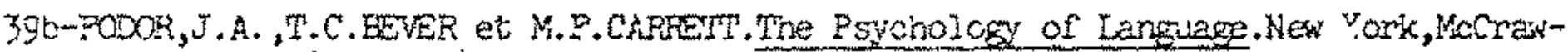
Hill, 1974 .

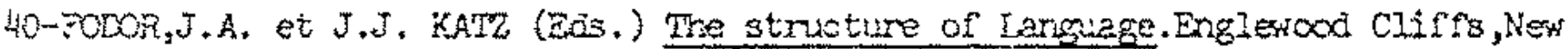
Jersey, Prentice-Hall, 1904 .

41-CARCIA, "Aluidiaries and the Critemion of Simplicity", in Ianguage,43 (1967), p. $853-870$.

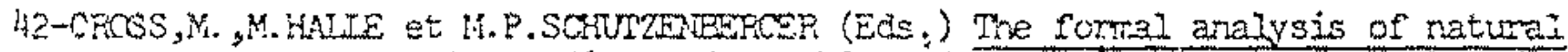
languages. Proceedins of the first tnternationel Conference. The Hague..., Moiton, 1973.

43-CPUIC, B. "Les theories transfomationnelles. Expose critique", in Ea Linguistique, $2(1965), p \cdot 1-24 ; 1(1969), p+31-101$.

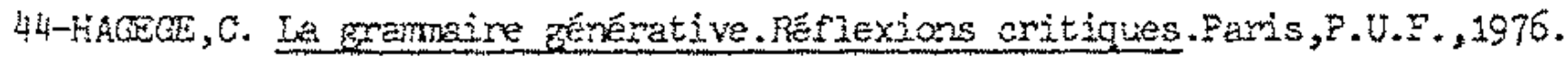

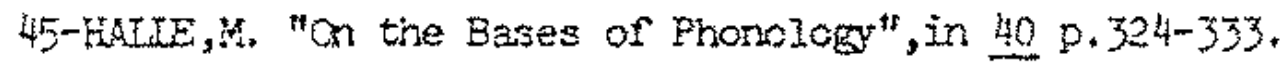

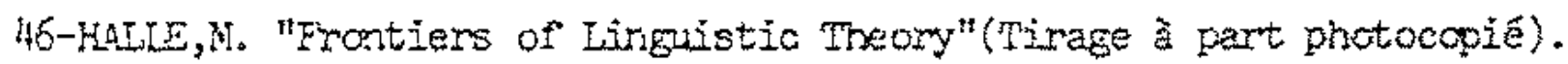

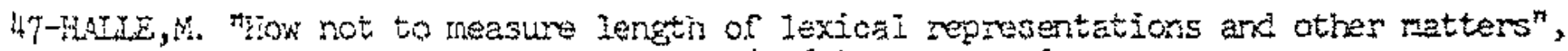
in Journal of Jirizustios, $5(2969), p .305-308$.

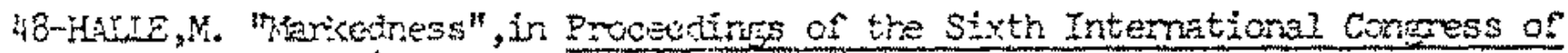
Phonetic Sciences, $p .61-72$.

49-HALE, M. "A Hew Organon, the Formalism of Phonolorical. fulles", in $420.1-11$.

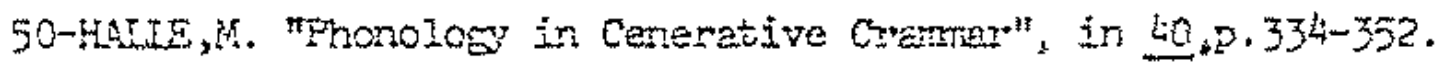

51-HRtE, H. "On the Role of Simplicity in Lingustis Descmitiors", in R.Jakooson (Ex.), Structure of Ensuage and its Natreratical Aspects. Providence, R.I., Anemcan Fkthematical Sockety, 1961,p.39-94.

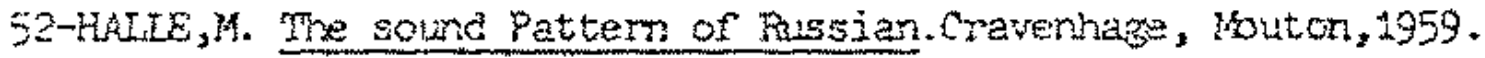

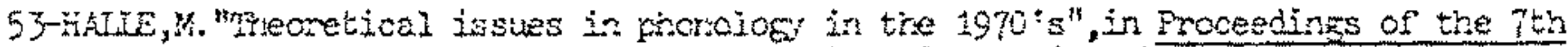
Intemational Congress of Phoretic sciences (1972),p.179-205.

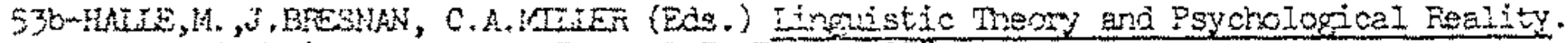

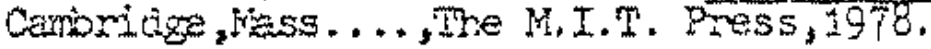

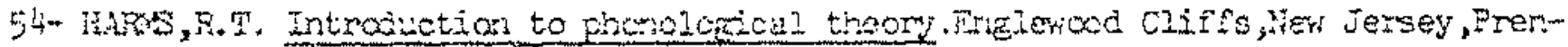
ticemplail, 2968 .

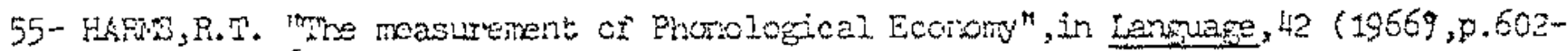
611.

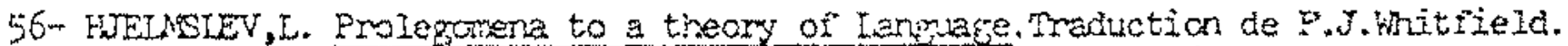
Madison, The unversity of Wisconsin Press, 1961.

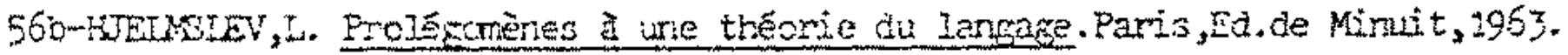

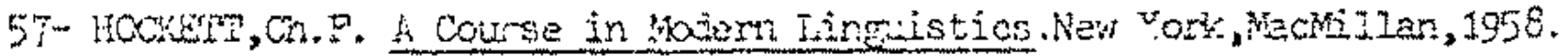

58- HOCNEIT, Ch. "A note on structure", in 71 p.279-280.

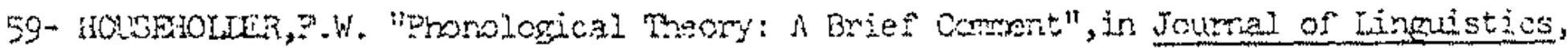
2 (1966), p.99-100. 


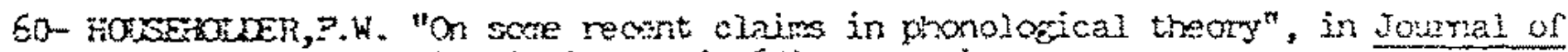
Lingisistics, 1 (1955), p. 13- zis.

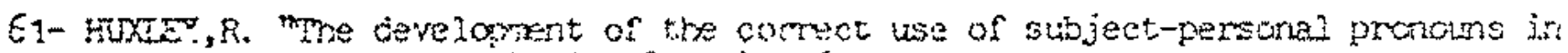
two chilaten", in 短 p.14:-165.

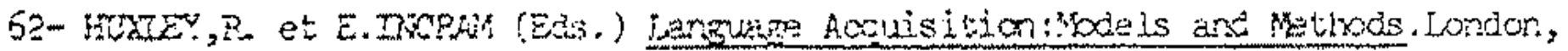
Acarientic Press, 1971 .

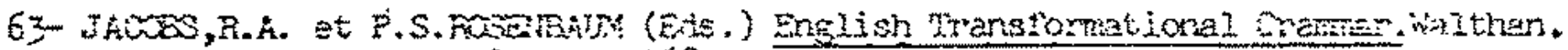
Hss., Biatsde1], 1968 .

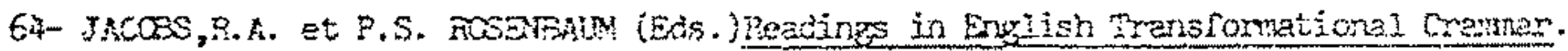
Helthan, Mess. , Cinn and Co.,1970.

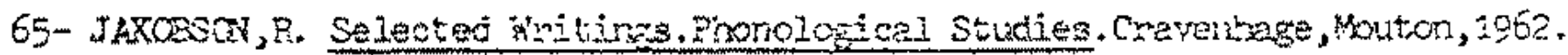

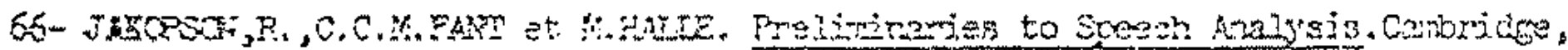

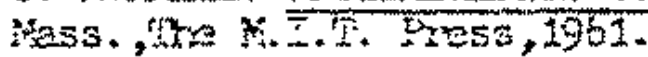

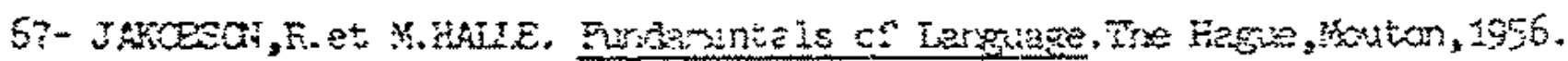

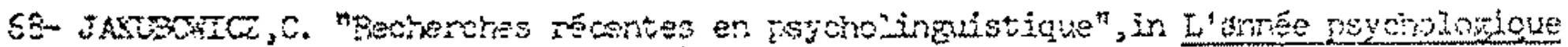
$70(1970), 101,0.247-293$.

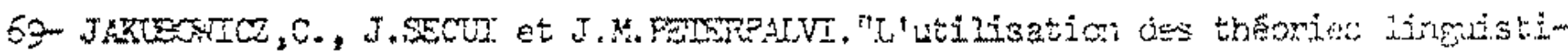

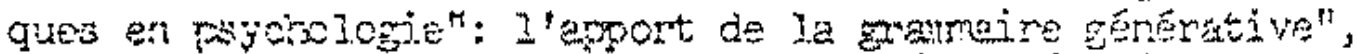
in Eulletin de Jsyctolope, 276(1968-1969),p.685-695.

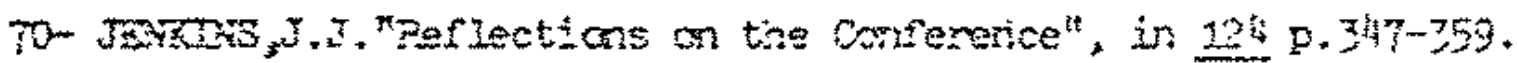

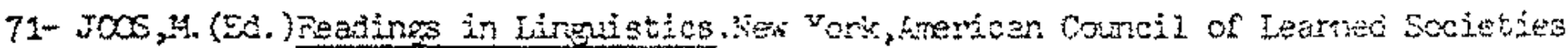
1957.

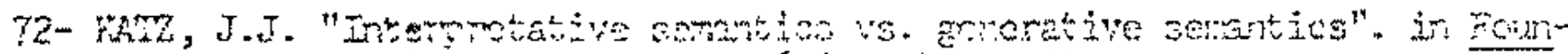

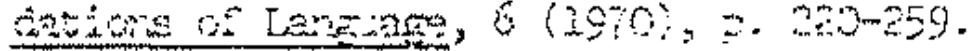

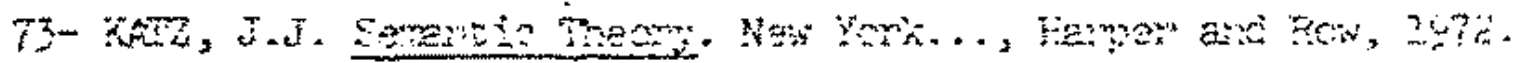

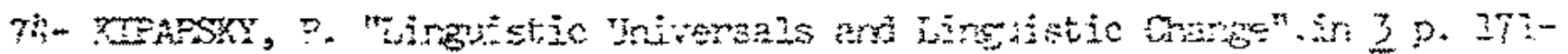
210 .

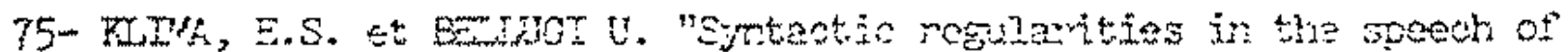
childurer" - in 82 . $183-219$.

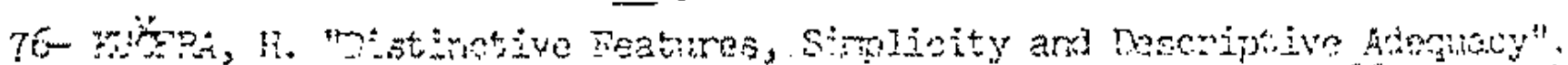

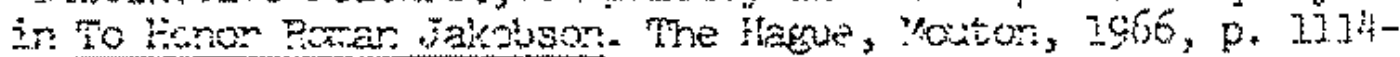
1235.

7T- LAKOF, G. "Pronotinalization, Yegstion and the Analysis of Aivents". in 64 p. $145-165$.

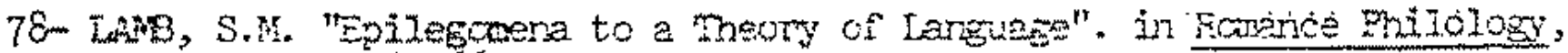
$29(1066)$, p. 531-57\%.

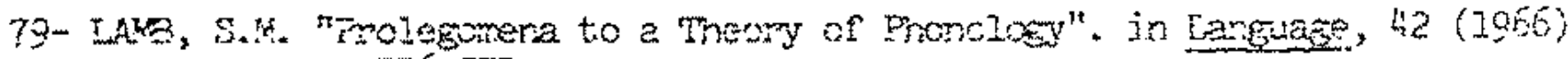
p. 530573.

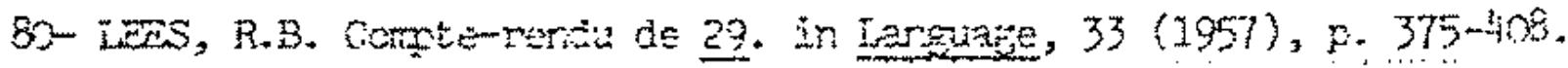

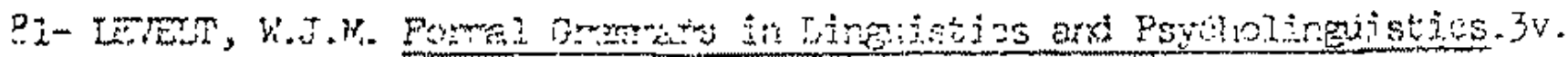

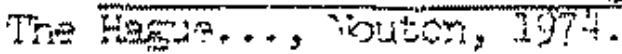

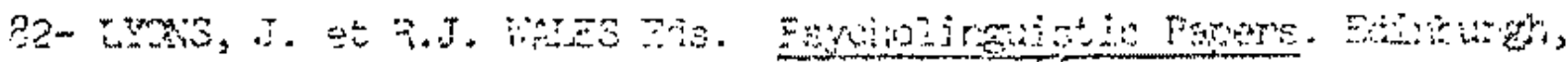

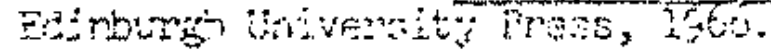

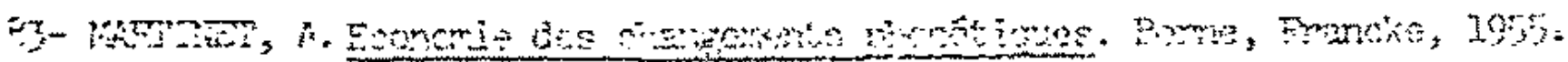

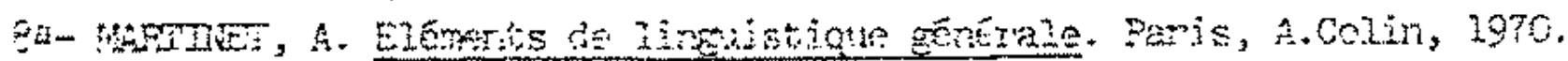


85-MARTDES, A. La linguistigue smohronioue. Paris, P.U.F., 1965.

96- MARTNET, A. "Au sujet des fondenents de la theorie inguisticue de

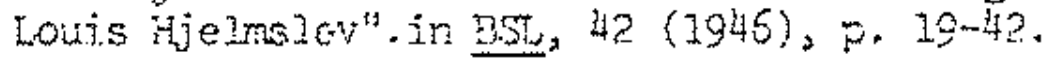

87- MATHEWS, P.H. "Some remanks ch the Householfor-tJalle contro-versy". In Joumal of Lingustics, 4 (1968), t. 275-283.

88- McCamey, J.D. "On the role of notatson in genertive phonolog," in 42 p. $51-62$.

89- MCawley, J.D. "Where Do Noun Phrases Cone From ?" in 64 p.166m-383.

go- MoveIf, D. The Acquisition of Denguage : The Study of Develommenta? Psycholr guistic. Wew York, derper and ho, $197 \%$

91- Metert, D. "Are there somificaliy linguistic universals ?" in 128 p. $530-535$.

22- Movitu, D. "Th Capacity fon the Ontogeresis of gremran". In 1.20 p. $17-40$.

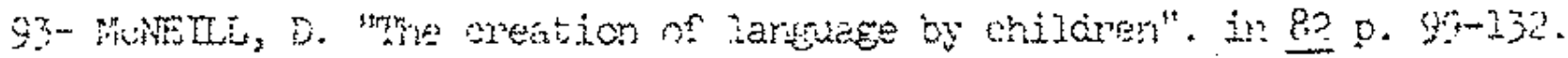

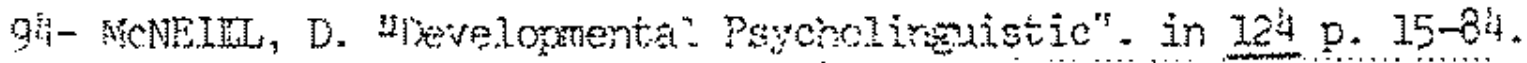

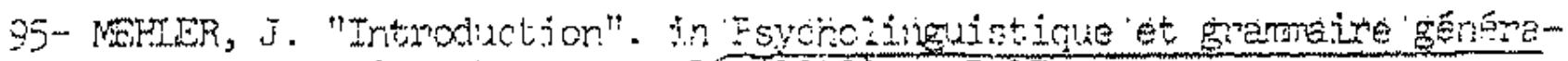
tive, tangagess, 16 (2969), p.3-15.

96- MERER, $J$. "Studies in Languge ard Thought Development". in $62 \mathrm{p}$. 201229.

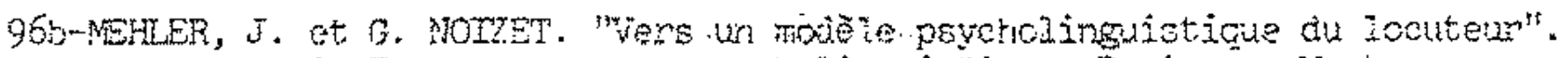
in Textes pou ure psyotiolinguistique. Faris..., Fouton, $1974, p \cdot 7-22$.

97- Motnm, G. La lirgoistique du we jecle. Paris, P.U.F., I972.

98- PALERM, D.S. "On Leamine to Talk : Are Principles Derived from the Iesming Labonatcry Applicable ?". in 120 p. AI-62.

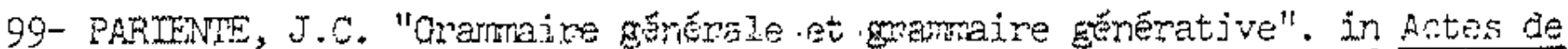
la recherche er sciences socieles, $5 / 6(1975), \mathrm{p}$. 36-i49.

100- PETERS, S. EO. Goals of Jingiristic theory. Englewood Cliffs, New Jersey, Feentice-Hall, 1972.

101- PसrLRS, S. "The projection problem : How is a gramar to be selected". in 100 p. $171-187$.

102- FEITRS, P.S. et R.W. RITCHTE. "On the Gererative Eower of Thansformation nal Grammars ". In Triformation Sciences; 6 (1973), p. $49-83$.

103-PEIERS,P.S. et R.W. RITCHE. "A Note on the Universal Base Hypothesis". in Sournal of Lingidistics, 5 (1969), p. $150 \mathrm{~m} 152$.

104-PETIRS, P.S. et, R.W. RITCHIE. "On restminting, the Base Component of Transfomational Gramarg". In Infomativi tra Cortmol. $20(1971)$, D. 487,503 .

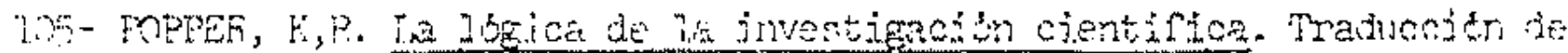

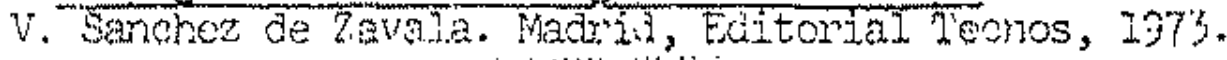

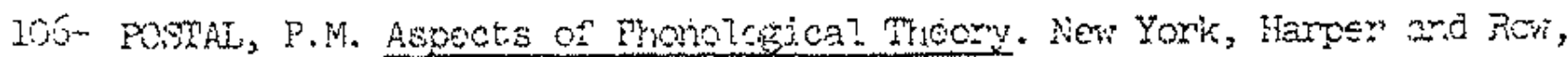
1908 . 
107- POSTAL, P.M. "Ithe Best Theory". in 100 p. 131-170.

108- POSTAL, P.M. "On the Surface Verb "Remind". in C.J. Fillmore et D. Terence Langendoen Eds. Studies in Iingtistic Semantics. New York... Holt, Rinehart and Winston, 1971, p. $181-270$.

109- POITIER, B. "La gramaire générstive et la Iinguistique". in Travaux de linguistique et de litterature, Université de Strasbourg, VI $(1968), 0.7-26$.

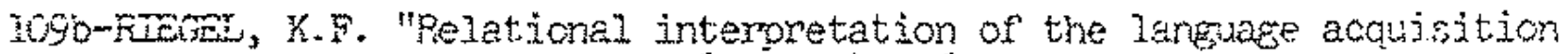
process". in $38 \mathrm{p}$. 224-236.

110- ROSS, J.R. "On Declarative Sentences". in 64 p. 222-272.

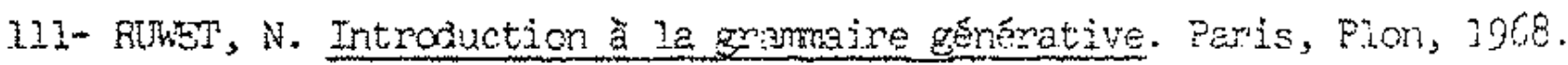

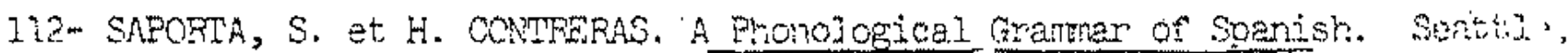
University of Washington Press, 2962.

113- SAUSSURE, F. dé. Cours de Inguistique generaje. Paris, Fayot, 1968.

214- SCHANE, S.S. "The formalization of exceptions in phonology". in 42 p. $63-70$.

115- SCHANE S.A. Generat ve pholology. Englewood Culff's, New Jersey, Prentice Hall, 1973 .

116- SCHEIESINGER, T.M. "Learning gramar : From Fivot to healization Rule". in 62 p. $79-93$.

117- SCHEIESTIGER, I.M. "Production of Utterances and Language Acquisition". in 120 p. $63-101$.

IITb-STERTSEMA, B. A Study of Glossematica. 2è ed. The Hague, Martinus Nijhoff, 3965 .

118- SDNCLAIR, H. "Sensorimotor Actions Pattems as a Condition for the Acquisition of Symtax". in 62 p. $121-130$.

119- SLOBIN, D.I. "Data for the Symposium". in 120 p. 3-14.

120-SLOBIN, D.I. Ed. The Critogenests of Gramar. New York..., Acarenit Press, 7971 .

i21- SIOBIN, D.I. "Seven questions about Language Develoment". in P.C. Dodwell EA. ; New Gortzons in PSychology. Penguin Education, 1972, p. 197-215.

122w SLOBIN, D.I. "Universals of gxametical development in children". in 38 p. $174 m 186$.

123- SLOBIN, D.I. et C'A. FERGUSON' Eds. 'Studies of: Child Langiage Development. New York, Holt, Rinehart et Winston, 1972.

124-SMTH, F. et G.A. MLJER Eds. The Genesis of Language. Cambridge, Mess., The M.I.T. Press, 1966 .

I24b-SOBER, E. Sirmilicity. Oxford, Clarendom Press, 1975.

225- SPANG-HANSSEN, $k$. "On the Simplicity of Descriptions". ir" Recherches Structurales, TCL $5(1949)$, p. 61-71. 
126- BTARS, A.W. "Linguistic-Mentallstic theory vowus an Explanatory S-R Leaming Theory of Ianguage Development". in 120 p. $103-150$.

127- SPAMEY, R. "Rexumancy rules in phonology". in Language 43 (2967), p. $393-435$.

128-STENJAER, D.E et L.A. JAROBOVITS Eds. Semantics. An Interdisciplinary Reader in Phi Iosophy, Tuinguistice and Psychology. Cambridge, At The thiversity Press, 197 i.

129-MPRiBENZKOY, N.S. Principes de phonclogie. Traduction de J. Cantirieau. Faris, KIncksleck, 1957 .

130- TWADEL, W. FTREEMN. "Uses and ADuses of Symetry". in Second sexas conference on eroblems of dirgustic Aralys in in Engish. Austin, Texas, The University of mexas, 1962, D. $129-137$.

13I- ULDAL; H.J. Quttine of glossematics. Part I : Generai Theory. Copenhagen, Nordisk Sprog-og Kulurforlag, 1957.

132- Y2N, S.L. "Two Measures of Econcryy in Fhonological Description" in Foumdations of Language, 4 (1968), p. 58-69.

33- ZIMEER, K.E. "On the evaluation of altermative phonological descriotions". in 'Joumizal of Linguistics, 6 (1969), p. 89-98. 